\title{
Renormalization group flow of the Luttinger-Ward functional: conserving approximations and application to the Anderson impurity model
}

\author{
J.F. Rentrop, V. Meden and S.G. Jakobs \\ Institut für Theorie der Statistischen Physik, RWTH Aachen University and \\ JARA - Fundamentals of Future Information Technology, 52062 Aachen, Germany
}

\begin{abstract}
We study the renormalization group flow of the Luttinger-Ward functional and of its two-particle irreducible vertex functions, given a cut-off in the two-particle interaction. We derive a conserving approximation to the flow and relate it to the fluctuation exchange approximation as well as to non-conserving approximations introduced in an earlier publication ctuation exchange approximation as well as to nonconserving approximations introducen [J. F. Rentrop, S. G. Jakobs, and V. Meden, J. Phys. A: Math. Theor. 48, 145002 (2015)]. We apply the different approximate flow equations to the single impurity Anderson model in thermal equilibrium at vanishing temperature. Numerical results for the effective mass, the spin susceptibility, the charge susceptibility, and the linear conductance reflect the similarity of the methods to the fluctuation exchange approximation. We find the majority of the approximations to deviate stronger from the exact results than oneparticle irreducible functional renormalization group schemes. However, we identify a simple static two-particle irreducible flow scheme which performs remarkably well and produces an exponential Kondo-like scale in the renormalized level position.

PACS numbers: 05.10.Cc, 11.10.Hi,71.10.-w,71.27.+a,73.21.La
\end{abstract}

\section{INTRODUCTION}

Baym and Kadanoff start the abstract of their seminal paper on how to construct conserving approximations to many-particle Green functions with the words "in describing transport phenomena, it is vital to build the conservation laws of number, energy, momentum, and angular momentum into the structure of the approximation" ! 1 For the following decades it was indeed a paradigm that approximate solutions to quantum many-body problems ought to be conserving. However, for low-dimensional systems known conserving approximation schemes suffer from severe artifacts. The conserving self-consistent Hartree-Fock approximation, for example, predicts an unphysical breaking of spin symmetry for the single impurity Anderson model at moderate interactions, ${ }^{[2}$ and it wrongly predicts the formation of a charge density wave in one-dimensional quantum wires with weak repulsive interaction $\frac{3}{3}$ As another example, two-particle Green functions computed with conserving approximations as proposed by Baym and Kadanofli14 violate the Pauli principle in form of the crossing symmetry relation. 5 -7

Maintaining conservation laws is usually not in the focus of renormalization group (RG) approaches to quantum many-body problems. Typical RG-based approximations are non-conserving, for instance standard truncations of the "functional" (or "exact") RG (fRG) in the one-particle irreducible (1PI) vertex expansion! ${ }^{819}$ The relation between fRG approximations and conservation laws was repeatedly under investigation. In particular the connection of the fRG to Ward identities attracted interest; Ward identities are relations between many-body correlation functions which encode the respective conservation laws. Katanin showed how the deviation of 1PI fRG results from Ward identities can be reduced by modifications in the truncation procedure ${ }^{8}$ Enss found the commonly employed fRG truncation schemes to be in principle incompatible with the Ward-identities typically used in the condensed-matter literature ${ }^{10}$ Kopietz and coworkers proceeded reversely and used Ward identities to create new truncations of the hierarchy of fRG flow equations. $11-13$

Another topic that raised attention in this context is the relation of the fRG to the conserving approximations proposed by Baym and Kadanoff. ${ }^{114}$ These are often called " $\Phi$-derivable" in reference to their construction. First an approximation to the Luttinger-Ward functiona ${ }^{14} \Phi$ is devised which is invariant under the symmetry transformations associated with the conservation law. Then correlation functions are computed from the twoparticle irreducible (2PI) vertex functions of $\Phi$, the physical value being determined by a self-consistency equation for the self-energy. It was shown for a scalar field theory that the physical self-energy of any given $\Phi$-derivable approximation can be obtained from a 1PI fRG flow, 15 for that purpose one expresses the 1PI four-point function that enters the flow equation via the $2 \mathrm{PI}$ four-point function that corresponds to the given approximation. This finding highlights the renormalizability of the vertex functions in $\Phi$-derivable approximations, which was studied intensively already before, see Ref. 16 and references therein. In Ref. 17, it is discussed how the $\Phi$-derivable approximation based on the second order approximation to $\Phi$ can be obtained from a 2PI fRG flow. References 15 and 17 thus show how a given $\Phi$-derivable approximation can be reconstructed by the fRG. In this paper we address the opposite question. Can the fRG be used to construct new $\Phi$-derivable approximations? So far, the required invariant approximate functional $\Phi$ is usually given by some subset of (skeleton) diagrams from the expansion of $\Phi$ in powers of the interaction. ${ }^{4}$ The fRG could be used to construct completely new, non-diagrammatic 
invariant approximations to $\Phi$. The precise form of the functional might even not be required if the fRG describes the flow of the corresponding physical values of the vertex functions.

A natural starting point for our investigation is the fRG flow of the Luttinger-Ward functional and of its vertex functions as described in Refs. 1819 and 20. In Ref. 20, we used the notions of " $C$-flow" and " $U$-flow" in order to distinguish whether the flow parameter is introduced into the free propagator $C$ or into the twoparticle interaction $U$. We showed that the hierarchy of $C$-flow equations for the physical vertex functions truncated straightforwardly at level $2 m$ is solved by $m$-th order self-consistent perturbation theory. This generalizes the result of Ref. [17 to arbitrary order (however only for condensed-matter problems without ultra-violet divergencies). Truncated $C$-flow is completely equivalent to the well known $\Phi$-derivable self-consistent perturbation theory. In particular, the result does not depend in any form on the choice of the flow parameter and its possibly regularizing properties. For models with infrared divergencies in perturbation theory, the straightforward application of $C$-flow RG is only possible if self-consistency has a regularizing effect.

Concerning the $U$-flow, we did not discuss in Ref. 20 how the truncation schemes relate to conserving approximations; we do so in this paper. Here, we show how $\Phi$-derivable approximations to the $U$-flow can be constructed. We carry out the relevant steps in a nontrivial truncation and find an approximation which is closely related to the fluctuation exchange (FLEX) approximation. 21 The corresponding invariant approximations to $\Phi$ are diagrammatically equivalent except for prefactors. Furthermore we study the $U$-flow of the physical vertex functions and identify the $U$-flow approximations from Ref. 20 as non- $\Phi$-derivable approximations to our $\Phi$-derivable one. Additionally, in the lowest order truncation we find a static non- $\Phi$-derivable approximate $U$-flow that was overlooked in Ref. 20 and which turns out to be remarkably accurate for the Anderson impurity model.

We also consider the combined $C$ - and $U$-flow. We find that the corresponding $\Phi$-derivable fRG approximations are identical to those of the pure $U$-flow. Furthermore we construct a non- $\Phi$-derivable combined $C$ - and $U$-flow approximation for the flow of the physical values with a parameter that allows to smoothly interpolate between pure $C$-flow and pure $U$-flow approximations. At a suitably chosen parameter value the range of applicability of the combined method to the Anderson impurity model is slightly larger than that of the pure $U$-fow.

We apply all our conserving and non-conserving approximations to the Anderson impurity model in equilibrium and study the effective mass, the spin susceptibility, the charge susceptibility and the linear conductance. In this way, we provide a comprehensive application of 2PI fRG approximations to a condensed matter quantum many-body problem.
In Ref. 20 we studied the performance of $2 \mathrm{PI}$ fRG approximations on the toy model of the anharmonic oscillator. There we identified a non- $\Phi$-derivable "modified" variant of the $U$-flow going back to Ref. 19 as more precise and faster than 1PI fRG with flowing four-point vertex; the modified $U$-flow variant has the self-consistent Hartree-Fock approximation as starting point which provides already a fairly good approximation for the case of the anharmonic oscillator. The question arises as to whether the high efficiency of this flow scheme pertains as well to actual many-body problems. The Anderson impurity model provides a test of particular interest, since self-consistent Hartree-Fock predicts for this model an unphysical breaking of spin symmetry at increased interactions. Does the modified $U$-flow restore the symmetry which is violated in its initial conditions? In this paper we show that this is not the case and that the modified $U$-flow performs comparably bad. Furthermore, we prove the plain and modified $U$-flow approximations of Ref. 20 to be non- $\Phi$-derivable by comparing numerical results for the dot occupancy obtained from different approaches. Consequently, there is no reason to expect these methods to preserve conservation laws; therefore, we will frequently refer to them as non-conserving methods.

Concerning our $\Phi$-derivable and thus conserving approximation to the $U$-flow, the numerical results turn out to be quite similar to those of the FLEX approximation for all studied observables. In particular, the effective mass is quickly overestimated as $U$ increases. There exists an analytic prediction ${ }^{22}$ that another approximation similar to FLEX produces a characteristic temperature scale $\sim \exp \left(-c U^{2}\right)$ [as opposed to the correct Kondo temperature $\left.\sim \exp \left(-c^{\prime} U\right)\right]$. The consequent presumption that our approximate effective mass correspondingly shows an $\exp \left(c U^{2}\right)$-behavior is however not confirmed by the data.

The paper is organized as follows: In Sec. II we briefly repeat the notation and the main definitions of Ref. 20. Section III then summarizes important aspects of $\Phi$ derivability discussed in the literature. In Sec. IV], we present our main analytical findings. We show how to obtain a conserving $U$-flow fRG scheme and specify its relation to FLEX. We then discuss how to view the $U$ flow schemes of Ref. 20 as approximations to the conserving $U$-flow. Moreover we show how to obtain a static (i.e. frequency-independent) $U$-flow scheme. We then describe an approximate 2PI fRG scheme that combines $C$ - and $U$-flow. In Sec. $\mathrm{V}$, we apply the different methods to the Anderson impurity model in equilibrium; for a concise presentation in the main part, we discuss many details in the appendices. We present numerical results for the Anderson model in Sec. VI which is followed by the concluding Sec. VII. Throughout the paper we set $\hbar=1$ and $k_{\mathrm{B}}=1$. 


\section{FERMIONIC 2PI FORMALISM: NOTATION AND DEFINITIONS}

In this paper we use the same notation and definitions as in Ref. 20. In this section, we only summarize the most important ones, restricting ourselves to the case of some fermionic many-body system. For details we refer to Ref. 20, in particular sections 2, 3 and 5. At the end of this section, we comment on the existence and uniqueness of the Luttinger-Ward functional.

We construct suitable generating functionals for equilibrium Green functions from the grand canonical partition function $Z[J]$ furnished with a source term. The source is chosen to be quadratic in the fields,

$$
Z[J]=\int D[\psi] e^{-S[\psi]+\frac{1}{2} \sum_{\alpha \alpha^{\prime}} \psi_{\alpha} J_{\alpha \alpha^{\prime}} \psi_{\alpha^{\prime}} .}
$$

Here, $\int D[\psi]$ is a functional integral over imaginary time Grassmann variables, and the action is given by

$$
\begin{aligned}
S[\psi]= & -\frac{1}{2} \sum_{\alpha \alpha^{\prime}} \psi_{\alpha}\left(C^{-1}\right)_{\alpha \alpha^{\prime}} \psi_{\alpha^{\prime}} \\
& +\frac{1}{4 !} \sum_{\alpha_{1} \alpha_{1}^{\prime} \alpha_{2} \alpha_{2}^{\prime}} U_{\alpha_{1} \alpha_{1}^{\prime} \alpha_{2} \alpha_{2}^{\prime}} \psi_{\alpha_{1}} \psi_{\alpha_{1}^{\prime}} \psi_{\alpha_{2}} \psi_{\alpha_{2}^{\prime}} .
\end{aligned}
$$

We use multi-indices $\alpha=(c, s, \tau)$. The charge index $c$ determines whether a field is creating $(+)$ or annihilating (-). For the Anderson model below, the state index $s$ will be the spin, $s=\sigma=\uparrow$ or $\downarrow$. As usual in thermal equilibrium, we can switch from imaginary times $\tau$ to Matsubara frequencies $\nu_{n}=\frac{\pi}{\beta}(2 n+1)$. One-particle quantities such as the free propagator $C$ are antisymmetric under exchange of the indices, $C_{\alpha \alpha^{\prime}}=-C_{\alpha^{\prime} \alpha}$. The two-particle interaction $U$ is fully antisymmetrized, $U_{\alpha_{1} \alpha_{2} \alpha_{3} \alpha_{4}}=\operatorname{sgn}(P) U_{\alpha_{P 1} \alpha_{P 2} \alpha_{P 3} \alpha_{P 4}}$ for any permutation $P$.

The charge index notation is advantageous for the methodological part of the paper. It allows for a compact notation with e.g. a single expression representing different channels of pair propagation. Furthermore, it applies in the same form to models which do or do not conserve particle number. We use it at the cost of obtaining at first unwieldy matrices with many zero components (which we then reduce to simpler objects) once we apply the formalism to the Anderson model with conserved particle number.

A Legendre transformation leads from $W[J]=\ln Z[J]$ to the $2 \mathrm{PI}$ effective action

$$
\Gamma[G]=\left.\{-W[J]-J \cdot G\}\right|_{J[G]},
$$

cf. Refs. 2324 and 25. The new independent variable is the full propagator $G$ with components $G_{\alpha \alpha^{\prime}}=$ $-\delta W / \delta J_{\alpha \alpha^{\prime}}=-\left\langle T \psi_{\alpha} \psi_{\alpha^{\prime}}\right\rangle_{J}$. In Eq. (3) we employed the dot product notation

$$
J \cdot G=\frac{1}{2} \sum_{\alpha \alpha^{\prime}} J_{\alpha \alpha^{\prime}} G_{\alpha \alpha^{\prime}}=\frac{1}{2} \sum_{\gamma} J_{\gamma} G_{\gamma}=-\frac{1}{2} \operatorname{tr} J G .
$$

Later we will use as well a trace based on the combined index $\gamma=\left(\alpha, \alpha^{\prime}\right)$,

$$
\operatorname{Tr} X=\frac{1}{2} \sum_{\gamma} X_{\gamma \gamma},
$$

which is to be distinguished from the single-index trace $\operatorname{tr} Y=\sum_{\alpha} Y_{\alpha \alpha}$. Furthermore, we will use the dot product inverse of a four-point function. It satisfies $(X$. $\left.X^{\text {inv }}\right)_{\gamma_{1} \gamma_{2}}=I_{\gamma_{1} \gamma_{2}}$. Here $I_{\gamma_{1} \gamma_{2}}=\delta_{\alpha_{1} \alpha_{2}} \delta_{\alpha_{1}^{\prime} \alpha_{2}^{\prime}}-\delta_{\alpha_{1} \alpha_{2}^{\prime}} \delta_{\alpha_{1}^{\prime} \alpha_{2}}$ is the neutral element with respect to the dot product, $X \cdot I=I \cdot X=X$.

The Luttinger-Ward functional is the difference between the 2PI effective action in the interacting and noninteracting case,

$$
\begin{aligned}
\Phi[G] & =\Gamma[G]-\Gamma_{0}[G] \\
& =\Gamma[G]-\frac{1}{2}\left[\operatorname{tr} \ln (-G)-\operatorname{tr}\left(C^{-1} G-1\right)\right] .
\end{aligned}
$$

Diagrammatically, it is given by minus the sum of all skeleton (2PI) diagrams contributing to the partition function, using full propagators as lines. Its functional derivative with respect to $G$ is minus the self-energy

$$
\Phi_{\gamma}^{(1)}[G]=\frac{\delta \Phi[G]}{\delta G_{\gamma}}=G_{\gamma}^{-1}-C_{\gamma}^{-1}-J_{\gamma}[G]=-\Sigma_{\gamma}[G] .
$$

We use a superscript " $(n)$ " to indicate the $n$-th functional derivative, for example $W_{\gamma_{1} \gamma_{2}}^{(2)}=\delta^{2} W / \delta J_{\gamma_{1}} \delta J_{\gamma_{2}}$. The derivatives of $\Phi$ and of other functionals obey the symmetry relations

$$
\begin{aligned}
\Phi_{\gamma_{1} \ldots \gamma_{n}}^{(n)} & =\Phi_{\gamma_{P 1} \ldots \gamma_{P n}}^{(n)}, \\
\Phi_{\gamma_{1} \ldots\left(\alpha_{i}, \alpha_{i}^{\prime}\right) \ldots \gamma_{n}}^{(n)} & =-\Phi_{\gamma_{1} \ldots\left(\alpha_{i}^{\prime}, \alpha_{i}\right) \ldots \gamma_{n}}^{(n)} .
\end{aligned}
$$

One obtains the physical quantities (denoted by a bar) by setting the external source $J$ to zero, for example $\bar{J}=$ $0, \bar{G}=G[\bar{J}], \bar{\Sigma}=-\bar{\Phi}^{(1)}=-\Phi^{(1)}[\bar{G}]$.

An important quantity is the pair propagator

$$
\Pi_{\gamma_{1} \gamma_{2}}[G]=-\frac{\delta G_{\gamma_{2}}}{\delta G_{\gamma_{1}}^{-1}}=G_{\alpha_{1}^{\prime} \alpha_{2}} G_{\alpha_{1} \alpha_{2}^{\prime}}-G_{\alpha_{1} \alpha_{2}} G_{\alpha_{1}^{\prime} \alpha_{2}^{\prime}} .
$$

It arises for instance in the flow Eq. 27) of $\Phi$ and in the Bethe-Salpeter equation

$$
W^{(2)}=\left(\Pi^{\mathrm{inv}}+\Phi^{(2)}\right)^{\mathrm{inv}}=\Pi-\Pi \cdot \Phi^{(2)} \cdot W^{(2)} .
$$

To conclude this section, let us briefly comment on the questions of existence and uniqueness of the LuttingerWard functional.

For some systems, the physical Green function has zeros, such that $\operatorname{det} \bar{G}=0$. Then, $\operatorname{tr} \ln (-\bar{G})=\ln \operatorname{det}(-\bar{G})$ in Eq. (6) is not defined and $\Phi[\bar{G}]$ does not exist; the formalism is not applicable in this case. This happens for gapped systems $\sqrt{26}$ which we do not investigate here.

The Legendre transformation in Eq. (3) requires the functional $J[G]$. A recent numerical study revealed the 
existence of a $\tilde{J} \neq 0$ with $G[\tilde{J}]=G[0]=\bar{G}$ for some models with on-site interaction. 27 This includes the Anderson model which we study below. This finding means that there exist two (or more) branches $J_{i}[G], i=1,2$ of the functional $J[G]$. Two branches $\Gamma_{i}[G]$ of the 2PI effective action arise, as well as two branches $\Phi_{i}[G]=$ $\Gamma_{i}[G]-\Gamma_{0}[G]$ of the Luttinger-Ward functional (the noninteracting $\Gamma_{0}[G]$ is unique) and two branches $\Sigma_{i}[G]=$ $-\Phi_{i}^{(1)}[G]=-G^{-1}+C^{-1}+J_{i}[G]$ of the self-energy functional. The physical state is correctly described by the branch which satisfies $J_{i}[\bar{G}]=0$ or, equivalently, the self-consistency equation $\Sigma_{i}[\bar{G}]=-\bar{G}^{-1}+C^{-1}$. Which branch does so may depend on the strength of the interaction, cf. Ref. 27 as well as Refs. 28 and 29 for toy model studies. On any branch, $\bar{G}$ is the only possible solution of the self-consistency equation $\Sigma_{i}[G]=-G^{-1}+C^{-1}$, since $J_{i}[G]=0$ entails $G=G\left[J_{i}[G]\right]=G[0]=\bar{G}$. Therefore, the self-consistency equation has the unique solution $\bar{G}$ on the physical branch and no solution on the other branches.

Below we study approximate functionals $\Phi^{\text {app }}[G]$ and $\Sigma^{\text {app }}[G]=-G^{-1}+C^{-1}+J^{\text {app }}[G]$. For these it may occur that the self-consistency equation $\Sigma^{\text {app }}[G]=-G^{-1}+C^{-1}$ has several solutions $\bar{G}_{j}^{\text {app }}$ with $J^{\text {app }}\left[\bar{G}_{j}^{\text {app }}\right]=0, j=$ $1,2, \ldots$. They indicate the existence of several branches of the functional $G_{j}^{\text {app }}[J]$. A prominent example are magnetic and non-magnetic solutions of the self-consistent Hartree-Fock approximation for the Anderson impurity model ${ }^{[2}$ All $\bar{G}_{j}^{\text {app }}=G_{j}^{\text {app }}[J=0]$ are approximations to the physical Green function $\bar{G}$ at vanishing external source $J$.

\section{T-DERIVABLE APPROXIMATIONS}

In Refs. 1 and 4, Baym and Kadanoff establish a method to construct a class of conserving approximations referred to as " $\Phi$-derivable". Here, we summarize those aspects of the method which are most relevant to devise and apply a conserving $2 \mathrm{PI}$ fRG approximation in the following sections.

A $\Phi$-derivable approximation is established in two steps. The first step is to set up an approximation to the Luttinger-Ward functional $\Phi[G]$ that is invariant under the relevant symmetry transformations of $G$. The second step is to determine the physical self-energy from a self-consistency equation.

Let us first discuss what it means if $\Phi$ is invariant under respective symmetry transformations of $G$. Let $\theta$ represent the parameters of the respective transformation. Then the invariance implies

$$
0=\frac{\delta}{\delta \theta} \Phi[G[\theta]]
$$

For an illustration, we switch to the notation of Ref. 4 in which real time arguments and no charge indices are used. In the case of particle number conservation, $\theta=$ $\theta(\vec{r}, t)$ and $G[\theta]$ is given by a gauge transformation

$$
G[\theta]_{\vec{r}, \vec{r}^{\prime}}\left(t, t^{\prime}\right)=e^{i \theta(\vec{r}, t)} G_{\vec{r}, \vec{r}^{\prime}}\left(t, t^{\prime}\right) e^{-i \theta\left(\vec{r}^{\prime}, t^{\prime}\right)} .
$$

Reference 4 is concerned with diagrammatic approximations to $\Phi[G]$ in terms of closed skeleton diagrams. For such approximations, the invariance of $\Phi[G[\theta]]$ results from a symmetry of the interaction vertices. For example, a density-density interaction is invariant under a gauge transformation,

$$
\begin{array}{r}
e^{-i \theta\left(\vec{r}_{1}^{\prime}, t\right)} e^{-i \theta\left(\vec{r}_{2}^{\prime}, t\right)}\left\langle\vec{r}_{1}^{\prime} \vec{r}_{2}^{\prime}|V| \vec{r}_{1} \vec{r}_{2}\right\rangle e^{i \theta\left(\vec{r}_{1}, t\right)} e^{i \theta\left(\vec{r}_{2}, t\right)} \\
=\left\langle\vec{r}_{1} \vec{r}_{2}^{\prime}|V| \vec{r}_{1} \vec{r}_{2}\right\rangle .
\end{array}
$$

Given a diagram to $\Phi$, one can combine each vertex with the transformations belonging to the ends of the attached propagator lines and obtain an invariant expression; this argument of Ref. 4 proves the invariance of $\Phi$. It can be formulated as well in charge index notation and with imaginary time arguments instead of real ones. Thus, a simple way to set up an invariant approximation to the Luttinger-Ward functional is to construct a diagrammatic approximation. Although this was not considered in Ref. 4, one can construct as well non-diagrammatic approximations to $\Phi[G]$ which are invariant.

Now, let us discuss the second step. Given an invariant approximate $\Phi[G]$, a conserving approximation results when the physical self-energy is determined from the self-consistency equation $\bar{\Sigma}=-\Phi^{(1)}[G[\bar{\Sigma}]]$, in which $G[\bar{\Sigma}]=\left(C^{-1}-\bar{\Sigma}\right)^{-1}$. The physical two-particle Green function $\bar{W}^{(2)}=-\delta G /\left.\delta J\right|_{J=0}$ can be obtained from $\bar{\Phi}^{(2)}$ via the Bethe-Salpeter equation 10 . (Baym and Kadanoff use a Bethe-Salpeter equation in the particlehole channel only ${ }^{1}$ ) Physical quantities computed from $\bar{\Sigma}$ and $\bar{W}^{(2)}$ respect conservation laws for particle number, momentum, and energy.

In Sec. II we mentioned that for some models (including the Anderson impurity model) there exist unphysical branches of the Luttinger-Ward functional. By solving the self-consistency equation one ensures that $\Phi$ derivable approximations are indeed always on the physical branch.

A problem of $\Phi$-derivable approximations is that their two-particle functions violate the crossing symmetry $[5]+7$ The exact solution for $W^{(2)}$ obeys the crossing symmetry relation

$$
\begin{aligned}
\left(W^{(2)}-\Pi\right)_{\alpha_{1} \alpha_{2} \alpha_{3} \alpha_{4}} & \\
= & \operatorname{sgn}(P)\left(W^{(2)}-\Pi\right)_{\alpha_{P 1} \alpha_{P 2} \alpha_{P 3} \alpha_{P 4}} .
\end{aligned}
$$

for any $P$. This relation is a consequence of the anticommutativity of fermionic field operators; it can hence be considered as a manifestation of the Pauli principle. By comparing different channels of the BetheSalpeter equation 10 one can show that typical $\Phi$ derivable approximations violate the crossing symmetry. 
For instance, crossing symmetry is broken in the selfconsistent Hartree Fock and in the FLEX approximation, and also in the conserving flow scheme which we derive in Sec. IV A. In our application to the Anderson model below we avoid the problem of violated crossing symmetry by studying only quantities that can be derived from $\Sigma$ alone, without computing $W^{(2)}$.

In order to compute physical observables which directly benefit from the conserving nature of $\Phi$-derivable approximations, one usually needs to determine $W^{(2)}$. For example, suppose to split the lead of the Anderson model studied below into a right and a left one. Then we could compute a left and a right conductance in linear response from a four-point vertex like $\bar{W}^{(2)}$, and both conductance values would be equal in $\Phi$-derivable approximations. Although we here do not access $W^{(2)}$ and derived observables, we call all $\Phi$-derivable approximations discussed below "conserving". This is appropriate as such observables could be calculated, the conservation laws being guaranteed to hold (but the crossing symmetry being broken). $\Phi$-derivable approximations do not only preserve conservation laws. They have as well advantages for quantities that can be derived from the selfenergy alone. As examples we now describe that they maintain the equivalence of different approaches to the mean occupancy and that they preserve the Friedel sum rule. In the applicaton to the Anderson model below we return to these issues, see Sec. VE and Fig. 3(b).

Mean occupancy. The mean occupancy $\left\langle n_{s}\right\rangle_{J=0}=$ $\operatorname{Tr} a_{s}^{\dagger} a_{s} e^{-(H-\mu N) / T} / \bar{Z}$ of a single-particle state $s$ in the physical $(J=0)$ thermal equilibrium can on the one hand be computed from the imaginary time Green function with equal time arguments,

$$
\begin{aligned}
\bar{G}_{(-, \tau s)(+, \tau s)} & =-\left\langle T a_{s}(\tau) a_{s}^{\dagger}(\tau)\right\rangle_{J=0} \\
& =\left\langle a_{s}^{\dagger} a_{s}\right\rangle_{J=0}=\left\langle n_{s}\right\rangle_{J=0} .
\end{aligned}
$$

On the other hand, $\left\langle n_{s}\right\rangle_{J=0}$ can be computed from the grand potential $\bar{\Omega}=-T \ln \bar{Z}$. For this purpose we use a source term $\epsilon_{s} a_{s}^{\dagger} a_{s}$ in the Hamiltonian, which is either present on physical grounds or added as an auxiliary term. Given hence a Hamiltonian of the form $H=\epsilon_{s} a_{s}^{\dagger} a_{s}+H^{\prime}$, we find

$$
\begin{aligned}
\frac{d \bar{\Omega}}{d \epsilon_{s}} & =-\frac{T}{\bar{Z}} \frac{d}{d \epsilon_{s}} \operatorname{Tr} e^{-\left(\epsilon_{s} a_{s}^{\dagger} a_{s}+H^{\prime}-\mu N\right) / T} \\
& =\frac{1}{\bar{Z}} \operatorname{Tr} a_{s}^{\dagger} a_{s} e^{-(H-\mu N) / T}=\left\langle n_{s}\right\rangle_{J=0} .
\end{aligned}
$$

This holds even if $a_{s}^{\dagger} a_{s}$ and $H^{\prime}$ do not commute.

Equations (15) and (16) are equivalent; in an exact calculation, they would yield the same result. However, for approximate calculations, this is in general not guaranteed. Truncated $1 \mathrm{PI}$ fRG, for instance, was found to spuriously break the equivalence of the two equations in an application to the Anderson impurity model $[30$ For $\Phi$-derivable approximations, in contrast, the thermodynamic consistency proven in Sec. IV of Ref. 4 ensures that both ways to determine $\left\langle n_{s}\right\rangle_{J=0}$ yield the same result. We sketch the argument only briefly.

The parameter $\epsilon_{s}$ enters the generating functionals via the free propagator $C$. Therefore, the derivative $d \bar{\Omega} / d \epsilon_{s}$ is formally given by the fRG flow equation for $\bar{\Omega}$ with a flow parameter in $C$. This is Eq. (46) of Ref. 20] and reads in the present context

$$
\frac{d \bar{\Omega}}{d \epsilon_{s}}=T \bar{G} \cdot \frac{d C^{-1}}{d \epsilon_{s}} .
$$

Its validity depends on the self-consistency $\bar{\Sigma}=$ $-\Phi^{(1)}[G[\bar{\Sigma}]]$ which is satisfied by construction in $\Phi$ derivable approximations. We insert $d C_{++}^{-1} / d \epsilon_{s}=$ $d C_{--}^{-1} / d \epsilon_{s}=0, d C_{-+}^{-1} / d \epsilon_{s}=-d C_{+-}^{-1} / d \epsilon_{s}$ and

$$
\frac{d}{d \epsilon_{s}} C_{\left(+, \tau_{1} s_{1}\right)\left(-, \tau_{2} s_{2}\right)}^{-1}=-\delta\left(\tau_{1}-\tau_{2}\right) \delta_{s_{1} s_{2}} \delta_{s_{1} s}
$$

to find

$$
\frac{d \bar{\Omega}}{d \epsilon_{s}}=\bar{G}_{(-, \tau s)(+, \tau s)}
$$

Therefore, Eqs. 15 and (16) are equivalent for $\Phi$ derivable approximations like the conserving fRG scheme from Sec. IV A below.

Friedel sum rule. As a second example we consider the Friedel sum rule. It holds for an impurity in a host at zero temperature and relates the scattering off the impurity to the charge displacement which it induces. In approximate calculations, the Friedel sum rule is not guaranteed to be preserved. Truncated 1PI fRG, for instance, was found to spuriously break the Friedel sum rule in an application to the Anderson impurity model $[30$ In contrast, $\Phi$-derivable approximations keep the Friedel sum rule valid, as explained now.

The rule was proven for interacting systems by Langer and Ambegaokar in Ref. 31. Their proof relies on the identity

$$
\operatorname{tr} \int d \nu e^{i \nu 0^{+}} \bar{G}_{-+}(i \nu) \frac{\partial \bar{\Sigma}_{+-}(i \nu)}{\partial \nu}=0,
$$

in which trace and matrix multiplication indicate a summation over single-particle states. The argument $\nu$ denotes the Matsubara frequency obtained by the usual Fourier transform [later we employ a different convention for the Fourier transform in Eqs. [60) and (61)]. Equation 20 in turn was proven by Luttinger and Ward in Ref. 14 by exploiting that the vertices in the diagrams to $\Phi$ conserve frequency. This is a consequence of the interaction being local in time and of the conservation of particle number. Equation 20 holds indeed in any $\Phi$-derivable approximation as long as the global gauge transformation

$$
G_{\left(c_{1} s_{1} \tau_{1}\right)\left(c_{2} s_{2} \tau_{2}\right)}(\theta)=e^{i c_{1} \tau_{1} \theta} G_{\left(c_{1} s_{1} \tau_{1}\right)\left(c_{2} s_{2} \tau_{2}\right)} e^{i c_{2} \tau_{2} \theta}
$$

leaves the approximate $\Phi[\bar{G}(\theta)]$ invariant,

$$
0=\left.\frac{d \Phi[\bar{G}(\theta)]}{d \theta}\right|_{\theta=0}=\left.\bar{\Phi}^{(1)} \cdot \frac{d \bar{G}(\theta)}{d \theta}\right|_{\theta=0} .
$$


In fact, as particle number conservation entails $\bar{G}_{++}=$ $\bar{G}_{--}=0$, this invariance equation can be written as

$$
\begin{aligned}
0=-\frac{i}{2} \sum_{c_{1} c_{2}} \sum_{s_{1} s_{2}} \int & d \tau_{1} d \tau_{2} \bar{\Sigma}_{\left(c_{1} s_{1} \tau_{1}\right)\left(c_{2} s_{2} \tau_{2}\right)} \\
& \times\left(c_{1} \tau_{1}+c_{2} \tau_{2}\right) \bar{G}_{\left(c_{1} s_{1} \tau_{1}\right)\left(c_{2} s_{2} \tau_{2}\right)} \\
= & i \operatorname{tr} \int d \tau_{1} d \tau_{2} \bar{\Sigma}_{+-}\left(\tau_{1}, \tau_{2}\right)\left(\tau_{1}-\tau_{2}\right) \bar{G}_{-+}\left(\tau_{2}, \tau_{1}\right) .
\end{aligned}
$$

This leads to

$$
\begin{gathered}
0=-\frac{1}{\beta^{2}} \operatorname{tr} \sum_{\nu_{n}, \nu_{m}} \bar{\Sigma}_{+-}\left(i \nu_{n}\right) \bar{G}_{-+}\left(i \nu_{m}\right) e^{i\left(\nu_{n}+\nu_{m}\right) 0^{+}} \\
\times \frac{\partial}{\partial \nu_{n}} \int_{-\beta / 2}^{\beta / 2} d \tau e^{-i\left(\nu_{n}-\nu_{m}\right) \tau} \\
\rightarrow-\operatorname{tr} \int \frac{d \nu_{1} d \nu_{2}}{2 \pi} \bar{\Sigma}_{+-}\left(i \nu_{1}\right) \bar{G}_{-+}\left(i \nu_{2}\right) e^{i\left(\nu_{1}+\nu_{2}\right) 0^{+}} \delta^{\prime}\left(\nu_{1}-\nu_{2}\right)
\end{gathered}
$$

in the limit $\beta=1 / T \rightarrow \infty$, which entails Eq. 207. We thus indirectly confirmed the validity of the Friedel sum rule in $\Phi$-derivable approximations like the conserving fRG scheme from Sec. IVA

The same reasoning holds for Luttinger's theorem. This theorem applies to bulk systems at zero temperature and states that the volume in momentum space in which the real part of the physical Green function at zero frequency is positive is given by the average particle number. Its derivation in Refs. 14 and 32 is based on the same identity (20) as used for the Friedel sum rule. Therefore, Luttinger's theorem is preserved in $\Phi$ derivable approximations.

The proof of Eq. 20) described above obviously requires the existence of the Luttinger-Ward functional. As mentioned in Sec. III there are systems for which the Luttinger-Ward functional does not exist. The Friedel sum rule and Luttinger's theorem may then by violated. Explicit examples for the breakdown of Luttinger's theorem are known. $26 \mid 33$

\section{CONSERVING AND NON-CONSERVING APPROXIMATIONS TO THE $U$-FLOW}

If a flow parameter $\lambda$ is introduced into the action, the $\lambda$-derivative of the Luttinger-Ward functional is given by an fRG flow equation. In Ref. 20, we focused on the hierarchy of flow equations that emerges for the physical vertex functions $\bar{\Phi}^{(n)}$. For the case that the flow parameter is introduced into the free propagator $C$ (" $C$-flow"), we proved the equivalence of the truncated hierarchy to the well-known conserving self-consistent perturbation theory. For the " $U$-flow", where the flow parameter enters instead the two-particle interaction $U$, we did not discuss how the truncation schemes relate to conserving approximations; we do so in this paper. We show how $\Phi$ derivable, conserving approximations can be constructed and how they are connected to the $U$-flow approximations used in Ref. 20.

In this Sec. IV we impose only few restrictions on the form in which the two-particle interaction $U_{\lambda}$ depends on the parameter $\lambda$ flowing from $\lambda_{\mathrm{i}}$ to $\lambda_{\mathrm{f}}$. First, we require that the interaction vanishes at the beginning of the flow, $U_{\lambda_{\mathrm{i}}}=0$. Consequently, the Luttinger-Ward functional and its vertex functions vanish there. Second, the original interacting problem is fully restored at the end of the flow, $U_{\lambda_{\mathrm{f}}}=U$. Finally, $U_{\lambda}$ has the same full index permutation antisymmetry as the bare interaction. In the discussion of the Anderson model below, we choose a multiplicative flow parameter $U_{\lambda}=\lambda U$ with $\lambda$ flowing from 0 to 1 . This simple choice of the flow parameter is sufficient; there is no need to regularize any divergence since perturbation theory in powers of $U$ is well behaved for the Anderson model ${ }^{34}$ When a flow of the propagator is considered in addition to the flow of the interaction, we introduce the flow parameter differently [cf. Eq. [530].

\section{A. Conserving approximations to the $U$-flow of $\Phi[G]$}

Let us construct conserving approximations to the $U$ flow. According to Eq. (77) of Ref. 20, the $U$-flow of the Luttinger-Ward functional is given by

$$
\dot{\Phi}_{\lambda}=\frac{1}{3 !} \operatorname{Tr} \dot{U}_{\lambda} \cdot\left[\left(\Pi^{\mathrm{inv}}+\Phi_{\lambda}^{(2)}\right)^{\mathrm{inv}}+\frac{\Pi}{2}\right],
$$

in which the dot denotes the derivative with respect to the flow parameter $\lambda$. This exact flow equation is the first of an infinite hierarchy: the flow of $\Phi_{\lambda}$ depends on $\Phi_{\lambda}^{(2)}$, that of $\Phi_{\lambda}^{(2)}$ involves $\Phi_{\lambda}^{(3)}$ and $\Phi_{\lambda}^{(4)}$, and so on.

In order to compute one-particle properties, we require an approximation to the physical value $\bar{\Sigma}=-\bar{\Phi}^{(1)}$ of the self-energy. According to Sec. III, a conserving approximation follows from the self-consistency equation $\bar{\Sigma}=-\Phi^{(1)}[G[\bar{\Sigma}]]$ if $\Phi$ satisfies the invariance equation $\delta \Phi[G[\theta]] / \delta \theta=0$. Let us hence study how one can obtain such an invariant $\Phi$ from a truncated flow equation.

The simplest truncation is to set $\Phi_{\lambda}^{(2)}=\Phi_{\lambda_{\mathrm{i}}}^{(2)}=0$ on the right-hand side of Eq. (27). The resulting flow equation reads as

$$
\dot{\Phi}_{\lambda}=\frac{1}{3 !} \operatorname{Tr} \dot{U}_{\lambda} \cdot \frac{3}{2} \Pi=\frac{1}{2} G \cdot \dot{U}_{\lambda} \cdot G .
$$

As the flow starts at $U=0$ and $\Phi=0$, the solution is

$$
\Phi=\frac{1}{2} G \cdot U \cdot G
$$

which is the first order perturbation theory result for the Luttinger Ward functional. The self-consistency equation $\bar{\Sigma}=-\bar{\Phi}^{(1)}=-U \cdot \bar{G}$ yields precisely the well known conserving Hartree-Fock approximation.

Now we consider the next higher order of truncation. We replace $\Phi_{\lambda}^{(2)}$ on the right-hand side of Eq. 27] by its 
leading perturbative value $U_{\lambda}$ (cf. also Sec. 6.2 of Ref. 20) ). The resulting flow equation is

$$
\begin{aligned}
\dot{\Phi}_{\lambda} & =\frac{1}{3 !} \operatorname{Tr} \dot{U}_{\lambda} \cdot\left[\frac{3}{2} \Pi+\Pi \cdot \sum_{k=1}^{\infty}\left(-U_{\lambda} \cdot \Pi\right)^{k}\right] \\
& =\frac{d}{d \lambda}\left[\frac{1}{4} \operatorname{Tr} U_{\lambda} \cdot \Pi-\frac{1}{3 !} \sum_{k=1}^{\infty} \frac{1}{k+1} \operatorname{Tr}\left(-U_{\lambda} \cdot \Pi\right)^{k+1}\right] .
\end{aligned}
$$

Here, $\left(U_{\lambda} \cdot \Pi\right)^{k}$ denotes the $k$-fold dot product $\left(U_{\lambda} \cdot \Pi\right)$. $\left(U_{\lambda} \cdot \Pi\right) \cdot \ldots \cdot\left(U_{\lambda} \cdot \Pi\right)$. If $U_{\lambda}$ has the same invariance under symmetry transformations as $U$, then $\delta \dot{\Phi}_{\lambda}[G[\theta]] / \delta \theta=0$. To see this one can apply the same argument as used after Eq. (13): combine the vertices $\dot{U}_{\lambda}$ or $U_{\lambda}$ with the transformations belonging to the ends of the attached propagators $G[\theta]$ (hidden in $\Pi[G[\theta]]$ ) to invariant expressions. In this case, the invariance of $\Phi_{\lambda}$ is respected during all of the flow. If, however, $\dot{U}_{\lambda}$ does not have the symmetry, the invariance equation for $\Phi_{\lambda}$ is violated during the flow. Nevertheless, it is reestablished at the end of the flow by the solution

$$
\Phi^{\mathrm{cfRG}}=\frac{1}{4} \operatorname{Tr} U \cdot \Pi-\frac{1}{3 !} \sum_{k=2}^{\infty} \frac{1}{k} \operatorname{Tr}(-U \cdot \Pi)^{k} .
$$

We label this conserving approximation scheme by "cfRG" ${ }^{47} \Phi^{\mathrm{cfRG}}$ deviates from the exact LuttingerWard functional in order $U^{3} G^{6}$ and higher. The corresponding approximate self-energy functional $\Sigma^{\mathrm{cfRG}}[G]=$ $-\Phi^{\text {cfRG (1) }}[G]$ can be determined from the rule $\operatorname{Tr} A$. $\delta \Pi / \delta G=4 A^{\mathrm{R}} \cdot G$. Here, $A$ denotes any fourpoint function with the usual symmetries $A_{\alpha_{1} \alpha_{1}^{\prime} \alpha_{2} \alpha_{2}^{\prime}}=$ $A_{\alpha_{2} \alpha_{2}^{\prime} \alpha_{1} \alpha_{1}^{\prime}}=-A_{\alpha_{1}^{\prime} \alpha_{1} \alpha_{2} \alpha_{2}^{\prime}}$, and we defined $A^{\mathrm{R}}$ via

$$
A_{\alpha_{1} \alpha_{1}^{\prime} \alpha_{2} \alpha_{2}^{\prime}}^{\mathrm{R}}=A_{\alpha_{1} \alpha_{2} \alpha_{2}^{\prime} \alpha_{1}^{\prime}} \text {. }
$$

In $A^{\mathrm{R}} \cdot G, G$ connects one index from the left index pair of $A$ to one index from the right pair. Applying the differentiation rule yields

$$
\begin{aligned}
\Sigma^{\mathrm{cfRG}} & =-U^{\mathrm{R}} \cdot G-\frac{2}{3} \sum_{k=1}^{\infty}\left[(-U \cdot \Pi)^{k} \cdot U\right]^{\mathrm{R}} \cdot G \\
& =-U \cdot G+\frac{2}{3}(\Upsilon \cdot U)^{\mathrm{R}} \cdot G,
\end{aligned}
$$

in which we introduced $\Upsilon=-\sum_{k=1}^{\infty}(-U \cdot \Pi)^{k}$. When we insert $G=\left(C^{-1}-\Sigma^{\mathrm{cfRG}}\right)^{-1}$ and solve the resulting selfconsistency equation we obtain the physical value $\bar{\Sigma}^{\text {cfRG }}$. From $\Phi^{\mathrm{cfRG}}=\Phi_{\text {exact }}+\mathcal{O}\left(U^{3} G^{6}\right)$ follows that $\bar{\Sigma}^{\mathrm{cfRG}}$ comprises all diagrams from second order self-consistent perturbation theory: $\bar{\Sigma}^{\mathrm{cfRG}}=\bar{\Sigma}_{\text {exact }}+\mathcal{O}\left(U^{3} \bar{G}_{2 \mathrm{SCPT}}^{5}\right)$, where $\bar{G}_{2 \mathrm{SCPT}}$ denotes the full propagator of the physical state in second order self-consistent perturbation theory.

We do not discuss higher order conserving truncation schemes since their analytic structure becomes increasingly complicated. Also their numerical solutions are difficult to realize; as the flowing objects are functionals, their numerical sampling would require a grid in the infinite dimensional space of functions.

Apart from the $U$-flow scheme given by Eq. (27), we studied in Ref. 20 as well a modification originally developed in Ref. 19. In the definition of the modified variant, the first order contribution to $\Phi$ is excluded from the replacement $U \rightarrow U_{\lambda}$. As a consequence, the RG flow does not start at $\Phi_{\lambda_{\mathrm{i}}}=0$ but at the Hartree-Fock solution, $\Phi_{\lambda_{\mathrm{i}}}=\frac{1}{2} G \cdot U \cdot G$. If one truncates the corresponding flow equation for $\Phi_{\lambda}$ by setting $\Phi_{\lambda}^{(2)}=\Phi_{\lambda_{\mathrm{i}}}^{(2)}=U$, the final solution is again the conserving approximation $\Phi^{\mathrm{cfRG}}$ given in Eq. (32). Hence, both approaches in their respective truncations are equivalent.

\section{B. Similarity between the cfRG and the FLEX approximation}

The cfRG approximation of Sec. IVA is closely related to the FLEX approximation of Refs. 21 and 35 which was heavily used to study high temperature superconductivity $\sqrt[36]{6}$ The FLEX approximation is as well $\Phi$-derivable. The approximate Luttinger-Ward functional $\Phi^{\text {FLEX }}$ is computed from a series of diagrams that describe ringlike pair-propagation, see Fig. 11(a). The motivation for this approximation is to incorporate effects resulting from the exchange of spin, density and particleparticle fluctuations. Compared to the expansion of the exact Luttinger-Ward functional, the first missing diagram is of order $U^{4} G^{8}$.

Each of the diagrams in Fig. 1 represents several total index pairings according to the Wick theorem. A pairing $P$ that contributes to a diagram of order $U^{n} G^{2 n}$ has the value

$$
\frac{\operatorname{sgn}(P)(-1)^{n+1}}{n ! 4 !^{n}} U \ldots U G \ldots G,
$$

in which $U \ldots U G \ldots G$ is a short hand notation for the appropriate index contractions. Summing up all diagrammatic contributions leads to

$\Phi^{\mathrm{FLEX}}=\frac{1}{4} \operatorname{Tr} U \cdot \Pi-\frac{1}{12} \operatorname{Tr} U \cdot \Pi \cdot U \cdot \Pi-\frac{1}{2} \sum_{k=3}^{\infty} \frac{1}{k} \operatorname{Tr}(-U \cdot \Pi)^{k}$

[see Eqs. (148) - (151) of Ref. 37 for an expression in charge-index free notation]. The functional $\Phi^{\mathrm{cfRG}}$ from the cfRG approximation given in Eq. $\sqrt{32}$ is identical to $\Phi^{\text {FLEX }}$ except for a factor $\frac{1}{3}$ in front of all diagrams of third order in $U$ and higher. We conclude that $\Phi^{\mathrm{cfRG}}$ accounts precisely for the FLEX diagrams, including however only a part of the weight of the higher order diagrams, see Fig. 1(b). We verified explicitly that the missing $\frac{2}{3}$ of those diagrams are generated in the conserving fRG by terms which are neglected in our truncation scheme. 
(a)
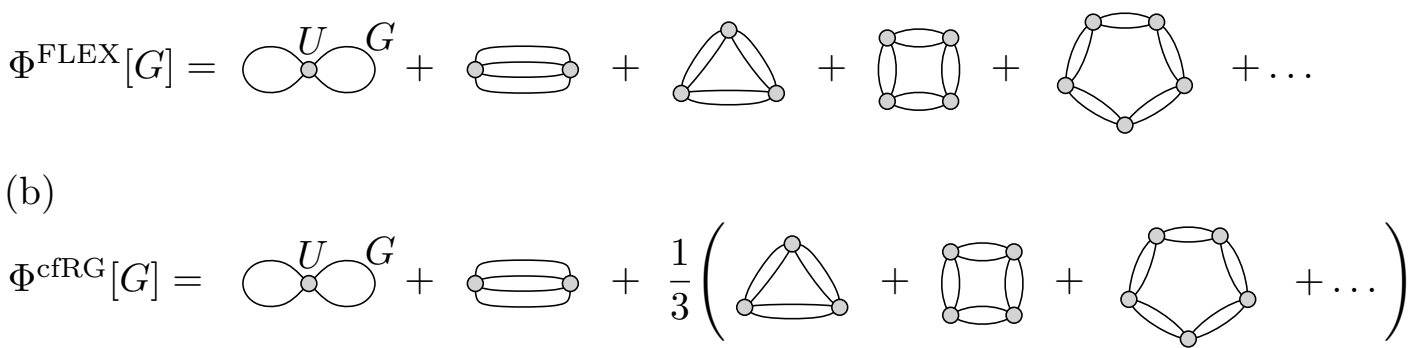

FIG. 1: (a) Diagrammatic representation of $\Phi^{\text {FLEX }}$. Our antisymmetrized charge index notation leads to diagrams with undirected lines and dot-like Hugenholtz vertices. When $\Phi^{\text {FLEX }}$ is expressed in terms of diagrams composed of directed propagator lines and Feynman interaction lines, one can see that it comprises three different channels: one with longitudinal spin fluctuations and density fluctuations, one with transverse spin fluctuations, and one with particle-particle fluctuations, cf. Refs. 21 and 35. (b) Diagrammatic representation of $\Phi^{\mathrm{cfRG}}$.

In FLEX, the self-consistency equation for the selfenergy reads

$$
\bar{\Sigma}^{\mathrm{FLEX}}=-U \cdot \bar{G}-\frac{4}{3}(U \cdot \bar{\Pi} \cdot U)^{\mathrm{R}} \cdot \bar{G}+2(\bar{\Upsilon} \cdot U)^{\mathrm{R}} \cdot \bar{G} .
$$

\section{Non-conserving approximations to the $U$-flow of $\bar{\Sigma}$}

Let us study how the cfRG approximation of Sec. IV A is related to the truncations of $U$-flow described in Ref. 20. Instead of computing the flow of the whole functional $\Phi_{\lambda}[G]$ we now consider only the flow of our quantity of interest, namely the physical value of the selfenergy $\bar{\Sigma}_{\lambda}=-\bar{\Phi}_{\lambda}^{(1)}$. The corresponding flow equation is

$$
\dot{\bar{\Sigma}}_{\lambda}=-\overline{\dot{\Phi}}_{\lambda}^{(1)}-\bar{\Phi}_{\lambda}^{(2)} \cdot \dot{\bar{G}}_{\lambda}
$$

in which according to Ref. 20

$$
\begin{aligned}
\dot{\Phi}_{\lambda}^{(1)}= & \frac{1}{3 !} \operatorname{Tr} \dot{U}_{\lambda} \cdot\left[\frac{1}{2} \frac{\delta \Pi}{\delta G}\right. \\
& \left.+W_{\lambda}^{(2)} \cdot\left(\Pi^{\mathrm{inv}} \cdot \frac{\delta \Pi}{\delta G} \cdot \Pi^{\mathrm{inv}}-\Phi_{\lambda}^{(3)}\right) \cdot W_{\lambda}^{(2)}\right], \\
\dot{\bar{G}}_{\lambda}= & \bar{\Pi}_{\lambda} \cdot \dot{\bar{\Sigma}}_{\lambda},
\end{aligned}
$$

with $W^{(2)}=\left(\Pi^{\mathrm{inv}}+\Phi^{(2)}\right)^{\text {inv }}$. The right-hand sides depend on $\bar{\Phi}_{\lambda}^{(2)}$ and $\bar{\Phi}_{\lambda}^{(3)}$. Nontrivial truncations of the flow Eq. 27$]$ for $\Phi_{\lambda}[G]$ produce approximate functionals $\Phi_{\lambda}[G]$ with non-vanishing $\bar{\Phi}_{\lambda}^{(n)} \neq 0, n \geq 1$, compare for example Eq. (32). [Only the most basic truncation Eq. 28) produces the Hartree-Fock solution with $\bar{\Phi}_{\lambda}^{(n)}=0$ for $n \geq 3$.] Consequently, we need $\bar{\Phi}_{\lambda}^{(2)}$ and $\bar{\Phi}_{\lambda}^{(3)}$ in order to determine the flow of $\bar{\Sigma}_{\lambda}$. However, the flow of $\bar{\Phi}_{\lambda}^{(2)}$ and $\bar{\Phi}_{\lambda}^{(3)}$ depends on higher $\bar{\Phi}_{\lambda}^{(n)}$, and so on. We face a new infinite hierarchy of coupled flow equations describing the flow of the physical values that correspond to the approximate $\Phi_{\lambda}[G]$.
Let us examine the idea to truncate as well the new hierarchy. In this way one obtains an approximation to the conserving approximation, which we expect to be in general non-conserving.

Consider for example the approximate flow of the functional $\Phi[G]$ described by Eq. 30]. It results from setting $\Phi_{\lambda}^{(2)}=U_{\lambda}$ on the right-hand side of Eq. (27). Let us hence truncate as well the new hierarchy for the physical values by setting $\bar{\Phi}_{\lambda}^{(2)}=U_{\lambda}, \bar{\Phi}_{\lambda}^{(3)}=0$ on the right-hand side of Eqs. (38) and (39). This leads precisely to the approximation scheme for the plain $U$-flow derived and used in Ref. 20. The resulting flow equation is

$$
\begin{aligned}
\dot{\bar{\Sigma}}_{\lambda}^{\mathrm{PUF}}=-\frac{2}{3}\left(\bar{\Upsilon}_{\lambda} \cdot \dot{U}_{\lambda} \cdot \bar{\Upsilon}_{\lambda}^{\mathrm{T}}\right. & \left.-\bar{\Upsilon}_{\lambda} \cdot \dot{U}_{\lambda}-\dot{U}_{\lambda} \cdot \bar{\Upsilon}_{\lambda}^{\mathrm{T}}\right)^{\mathrm{R}} \cdot \bar{G}^{\lambda} \\
& -\dot{U}_{\lambda} \cdot \bar{G}_{\lambda}-U_{\lambda} \cdot \dot{\bar{G}}_{\lambda}, \quad(41)
\end{aligned}
$$

with $\Upsilon_{\gamma_{1} \gamma_{2}}^{\mathrm{T}}=\Upsilon_{\gamma_{2} \gamma_{1}}$. We label this approximation scheme by "PUF". The corresponding initial conditions are $U_{\lambda_{\mathrm{i}}}=0$ and $\bar{\Sigma}_{\lambda_{\mathrm{i}}}^{\mathrm{PUF}}=0$. We have identified this scheme as a probably non-conserving approximation to the cfRG approximation of Sec. IVA. Numerical results for the impurity occupancy of the Anderson model show that the PUF approximation is non- $\Phi$-derivable, cf. Sec. VIC, We therefore expect possible extensions of this approximation scheme which access two-particle functions to be non-conserving.

The same strategy can be applied in the framework of the modified $U$-flow. Then it leads to the approximation for the modified $U$-flow derived and used in Ref. 20 . It obviously constitutes another non- $\Phi$-derivable approximation to the cfRG approximation. The corresponding flow equation is

$$
\begin{array}{r}
\dot{\bar{\Sigma}}_{\lambda}^{\mathrm{MUF}}=-\frac{2}{3}\left(\bar{\Upsilon}_{\lambda} \cdot \dot{U}_{\lambda} \cdot \bar{\Upsilon}_{\lambda}^{\mathrm{T}}-\bar{\Upsilon}_{\lambda} \cdot \dot{U}_{\lambda}-\dot{U}_{\lambda} \cdot \bar{\Upsilon}_{\lambda}^{\mathrm{T}}\right)^{\mathrm{R}} \cdot \bar{G}^{\lambda} \\
-U \cdot \dot{\bar{G}}_{\lambda}, \quad(42)
\end{array}
$$

with the self-consistent Hartree-Fock self-energy as starting point, $\bar{\Sigma}_{\lambda_{i}}^{\mathrm{MUF}}=\bar{\Sigma}^{\mathrm{HF}}=-U \cdot G\left[\bar{\Sigma}^{\mathrm{HF}}\right]$. We use the label "MUF" for this specific approximation. 
Let us apply the idea of a second truncation as well to the most basic truncation scheme from Eq. (28). This has been constructed by setting $\Phi_{\lambda}^{(2)}=0$ on the right-hand side of the flow equation (27) for the functional. Accordingly, we truncate the new hierarchy for the physical values by setting $\bar{\Phi}_{\lambda}^{(2)}=0, \bar{\Phi}_{\lambda}^{(3)}=0$ on the right-hand side of Eqs. (38) and (39). This leads to the flow equation

$$
\dot{\bar{\Sigma}}_{\lambda}^{\mathrm{StUF}}=-\dot{U}_{\lambda} \cdot \bar{G}
$$

with initial condition $\bar{\Sigma}_{\lambda_{i}}^{\text {StUF }}=0$. It provides a simple, static approximation to the physical self-energy, which we refer to as "StUF". The existence of this approximation was overlooked in Ref. 20 .

\section{Combined $C$ - and $U$-flow}

It was shown in Ref. 20 that straightforward truncations of $C$-flow lead to standard self-consistent perturbation theory. For the Anderson model which we study below, Ref. 38 provides data from second order self-consistent perturbation theory, equivalent to $C$-flow truncated at level 4 . We observe that these data typically deviate from the exact result in the opposite direction than those obtained by the $U$-flow methods from the previous sections. Therefore, we suspect that mixing both schemes could improve the approximation. The idea of introducing a flow parameter into both, $C$ and $U$, was already formulated in Ref. 19. Here, we refer to this approach as $C U$-flow.

The Luttinger-Ward functional $\Phi[G]$ does not depend on $C$. As a consequence, the flow equation for $\Phi_{\lambda}[G]$ in the $C U$-flow is identical to the $U$-flow case and given by Eq. 27). Accordingly, the conserving approximations to the $U$-flow of $\Phi[G]$ from Sec. IV A pertain as well to the $C U$-flow.

In contrast, new (non- $\Phi$-derivable) approximations arise for the $C U$-flow of the physical value of the selfenergy, $\dot{\bar{\Sigma}}_{\lambda}=-\bar{\Phi}_{\lambda}^{(1)}-\bar{\Phi}_{\lambda}^{(2)} \cdot \dot{\bar{G}}_{\lambda}$. While $\dot{\Phi}_{\lambda}^{(1)}$ is still given by Eq. 39, , $\dot{\bar{G}}_{\lambda}$ now satisfies

$$
\dot{\bar{G}}_{\lambda}=\bar{\Pi}_{\lambda} \cdot\left(\dot{\bar{\Sigma}}_{\lambda}-\dot{C}_{\lambda}^{-1}\right)
$$

instead of Eq. (40). Here, $\dot{C}_{\lambda}^{-1}$ denotes $d\left(C_{\lambda}^{-1}\right) / d \lambda$. Let us apply the same truncation as in Sec. IVC and set $\bar{\Phi}_{\lambda}^{(2)}=U_{\lambda}$ and $\bar{\Phi}_{\lambda}^{(3)}=0$ on the right-hand sides. This results in an equation that is formally identical to Eq. (41) from the $U$-flow, however with $\dot{\bar{G}}_{\lambda}$ now given by Eq. 444.

For the Anderson impurity model studied below it is known that second order perturbation theory provides good approximations for $U \ll \pi \Gamma, \sqrt[34]{,}$ with $\Gamma$ being a measure for the coupling between impurity level and lead. On that account we demand that the approximate $\bar{\Sigma}$ obtained from the truncated $C U$-flow is exact up to second order in $U$ (as are $\bar{\Sigma}^{\text {cfRG }}, \bar{\Sigma}^{\text {FLEX }}, \bar{\Sigma}^{\mathrm{PUF}}$ and $\left.\bar{\Sigma}^{\mathrm{MUF}}\right)$. The truncation described above does not satisfy this condition. The perturbative expansion of the exact physical value of the self-energy in the presence of a flow parameter is given by

$$
\bar{\Sigma}_{\lambda}^{\text {exact }}=D_{\lambda}^{1 \mathrm{st}}+D_{\lambda}^{2 \mathrm{ndHF}}+D_{\lambda}^{2 \text { ndS }}+\mathcal{O}\left(U^{3}\right),
$$

in which $D_{\lambda}^{1 \mathrm{st}}=-U_{\lambda} \cdot C_{\lambda}$ denotes the value of the first order diagram, $D_{\lambda}^{2 \text { ndHF }}=U_{\lambda} \cdot \Pi_{\lambda}^{0} \cdot U_{\lambda} \cdot C_{\lambda}$ that of the (non-skeleton) second order diagram contained in selfconsistent Hartree-Fock, and $D_{\lambda}^{2 \text { ndS }}=\frac{2}{3}\left(U_{\lambda} \cdot \Pi_{\lambda}^{0} \cdot U_{\lambda}\right)^{\mathrm{R}} \cdot C_{\lambda}$ that of the skeleton second order diagram. $\left[\Pi^{0}\right.$ is defined by Eq. (9) with $C$ replacing $G$.] One can show that the above truncation satisfies

$$
\begin{aligned}
& \dot{\bar{\Sigma}}_{\lambda, \gamma_{1}}=\dot{D}_{\lambda, \gamma_{1}}^{1 \mathrm{st}}+\dot{D}_{\lambda, \gamma_{1}}^{2 \mathrm{ndHF}} \\
& +\frac{2}{3}\left(U_{\lambda} \cdot \Pi_{\lambda}^{0} \cdot \dot{U}_{\lambda}+\dot{U}_{\lambda} \cdot \Pi_{\lambda}^{0} \cdot U_{\lambda}\right)^{\mathrm{R}} \cdot C_{\lambda}+\mathcal{O}\left(U_{\lambda}^{2} \dot{U}_{\lambda}, U_{\lambda}^{3}\right) .
\end{aligned}
$$

Obviously, the last addend of

$$
\begin{aligned}
\dot{D}_{\lambda}^{2 \text { ndS }}=\frac{2}{3}\left(U_{\lambda} \cdot \Pi_{\lambda}^{0} \cdot \dot{U}_{\lambda}\right. & \left.+\dot{U}_{\lambda} \cdot \Pi_{\lambda}^{0} \cdot U_{\lambda}\right)^{\mathrm{R}} \cdot C_{\lambda} \\
& +2\left(U_{\lambda} \cdot \Pi_{\lambda}^{0} \cdot U_{\lambda}\right)^{\mathrm{R}} \cdot \dot{C}_{\lambda}
\end{aligned}
$$

is missing.

Let us formulate a minimal extension of the above truncation scheme which makes $\bar{\Sigma}$ exact up to second order in $U$. As it is insufficient to truncate the flow equation by the first order approximation $\bar{\Phi}_{\lambda}^{(2)}=U_{\lambda}$, we consider the second order approximation,

$$
\begin{aligned}
& \left.\Phi_{\lambda}^{(2)}[G]\right|_{2 \text { nd }}=U_{\lambda}+V_{\lambda}[G] \\
& V_{\lambda}[G]=-\left(U_{\lambda} \cdot \Pi[G] \cdot U_{\lambda}\right)^{\mathrm{R}}-\left(U_{\lambda} \cdot \Pi[G] \cdot U_{\lambda}\right)^{\mathrm{R}^{\mathrm{R}}} .
\end{aligned}
$$

For $\bar{\Sigma}$ to be exact in second order, it is indeed sufficient to use $\bar{\Phi}_{\lambda}^{(2)}=\left.\bar{\Phi}_{\lambda}^{(2)}\right|_{2 \text { nd }}$ for one particular $\bar{\Phi}_{\lambda}^{(2)}$ in the flow equation only. The other vertex functions can be truncated as before by $\bar{\Phi}_{\lambda}^{(2)}=U_{\lambda}$ and $\bar{\Phi}_{\lambda}^{(3)}=0$. In this way, the numerical effort for solving the flow equations does not increase significantly. Specifically, we truncate the flow equation

$$
\dot{\bar{\Sigma}}_{\lambda}=-\bar{\Phi}_{\lambda}^{(1)}-\bar{\Phi}_{\lambda}^{(2)} \cdot \bar{\Pi}_{\lambda} \cdot \dot{\bar{\Sigma}}_{\lambda}+\bar{\Phi}_{\lambda}^{(2)} \cdot \bar{\Pi}_{\lambda} \cdot \dot{C}_{\lambda}^{-1}
$$

to

$$
\begin{aligned}
\dot{\bar{\Sigma}}_{\lambda}^{\mathrm{CUF}}= & -\left.\overline{\dot{\Phi}}_{\lambda}^{(1)}\right|_{\bar{\Phi}_{\lambda}^{(2)} \rightarrow U_{\lambda}, \bar{\Phi}^{(3)} \rightarrow 0} \\
& -U_{\lambda} \cdot \bar{\Pi}_{\lambda} \cdot \dot{\bar{\Sigma}}_{\lambda}^{\mathrm{CUF}}+\left.\bar{\Phi}_{\lambda}^{(2)}\right|_{2 \mathrm{nd}} \cdot \bar{\Pi}_{\lambda} \cdot \dot{C}_{\lambda}^{-1} .
\end{aligned}
$$

We label this approximation by "CUF". Compared to Eq. (46), it includes the additional addend

$$
\bar{V}_{\lambda} \cdot \bar{\Pi}_{\lambda} \cdot \dot{C}_{\lambda}^{-1}=2\left(U_{\lambda} \cdot \Pi_{\lambda}^{0} \cdot U_{\lambda}\right)^{\mathrm{R}} \cdot \dot{C}_{\lambda}+\mathcal{O}\left(U^{3}\right) .
$$

which contains indeed the missing part of $\dot{D}_{\lambda}^{2 \text { ndS }}$ from Eq. (47). 
So far we have discussed a combination of the $C$-flow with the plain $U$-flow. Likewise it is possible to combine the $C$-flow with the modified $U$-flow. As described in Ref. 19, the starting point of the flow is then $\bar{\Sigma}_{\lambda_{\mathrm{i}}}=0$, whereas the original modified $U$-flow intentionally starts at $\bar{\Sigma}_{\lambda_{\mathrm{i}}}=\bar{\Sigma}^{\mathrm{HF}}$. For the Anderson impurity model, we have implemented both, the combination of $C$-flow with plain and with modified $U$-flow. As the results are qualitatively similar, we do not present further details on the combination with modified $U$-flow.

Concerning the flow parameter, we combine a sharp infrared cut-off of the imaginary frequency in the free propagator with an exponential rescaling of the interaction amplitude,

$$
C_{\lambda}\left(\nu_{n}\right)=C\left(\nu_{n}\right) \theta\left(\left|\nu_{n}\right|-\lambda\right), \quad U_{\lambda}=e^{-\lambda / \Lambda} U,
$$

in which $\lambda$ flows from infinity to zero. The resulting initial conditions are $C_{\lambda_{\mathrm{i}}}=0, U_{\lambda_{\mathrm{i}}}=0, \bar{\Sigma}_{\lambda_{\mathrm{i}}}^{\mathrm{CUF}_{\Lambda}}=0$. The superscript "CUF ${ }_{\Lambda}$ " now comprises a reference to the constant $\Lambda>0$ which appears in Eq. (53). This constant determines how fast $U$ is turned on in comparison to $C$. Indeed, it allows to interpolate between the pure $U$-flow and $C$-flow methods. If $\Lambda$ is small, the largest part of the flow of the free propagator happens while the interaction is still negligibly small. Only then, given an almost completely restored propagator, $U$ flows to considerable values. Hence, we expect the method to produce data close to the $U$-flow result, $\bar{\Sigma}^{\mathrm{CUF}_{\Lambda}} \rightarrow \bar{\Sigma}^{\mathrm{PUF}}$ for $\Lambda \rightarrow 0$. If $\Lambda$ is large, we expect in turn results close to that of the pure $C$-flow. The scale $\Lambda_{0}$ which separates the two regimes depends on the model and is difficult to determine a priori. In the limiting case of infinite $\Lambda$, that is $U_{\lambda}=U$, we reproduce a pure $C$-flow, $\bar{\Sigma}^{\mathrm{CUF}}{ }_{\infty}=\bar{\Sigma}^{\mathrm{CF}}$, in which

$$
\dot{\bar{\Sigma}}_{\lambda}^{\mathrm{CF}}=-U \cdot \dot{\bar{G}}_{\lambda}+\left.\bar{V}_{\lambda}\right|_{U_{\lambda} \rightarrow U} \cdot \bar{\Pi}_{\lambda} \cdot \dot{C}_{\lambda}^{-1} .
$$

We refer to this specific approximation as "CF". The underlying truncation, which is partly based on the approximation $\bar{\Phi}_{\lambda}^{(2)}=U$ and partly on $\bar{\Phi}_{\lambda}^{(2)}=\left.\bar{\Phi}_{\lambda}^{(2)}\right|_{2 \text { nd }}$, is not among the truncations described in Ref. 20 and is not equivalent to self-consistent perturbation theory.

Numerical computations cannot start at $\lambda_{\mathrm{i}}=\infty$ but only at some finite $\lambda_{i}^{\text {num }}$. If $\lambda_{i}^{\text {num }}$ is chosen sufficiently large, the flow from $\lambda=\infty$ to $\lambda_{\mathrm{i}}^{\text {num }}$ does not contribute significantly to $\bar{\Sigma}^{\mathrm{CUF}_{\Lambda}}$. However, there is an important contribution to $\bar{\Sigma}^{\mathrm{CF}}$ given by

$$
\begin{aligned}
\bar{\Sigma}_{\lambda_{\mathrm{i}}^{\text {num }}}^{\mathrm{CF}} & =\lim _{\eta \rightarrow 0^{+}} \int_{\infty}^{\lambda_{\mathrm{i}}^{\text {num }}} d \lambda\left(-U \cdot \bar{G}_{\lambda}\right) \\
& \approx \lim _{\lambda_{0} \rightarrow \infty} \lim _{\eta \rightarrow 0^{+}} \int_{\infty}^{\lambda_{0}} d \lambda\left(-U \cdot \bar{G}_{\lambda}\right) .
\end{aligned}
$$

Here, $\eta$ is the infinitesimal shift of imaginary time which ensures that creators are ordered to the left of annihilators with equal time arguments. A similar contribution due to the flow from $\lambda=\infty$ to $\lambda_{i}^{\text {num }}$ is known from 1PI fRG with imaginary frequency cut-off, cf. e.g. Ref. 39.

\section{E. Summary of methods}

In the following sections we apply the different approximation schemes to the single impurity Anderson model. For a better overview, we list the methods that we have introduced: the conserving fRG approximation $\bar{\Sigma}^{\text {cfRG }}$ which is the self-consistent solution of Eq. (34), and the FLEX approximation $\bar{\Sigma}^{\text {FLEX }}$ which follows from Eq. (37); furthermore, as non-conserving approximations to $\bar{\Sigma}^{\text {ctRG }}$, the plain $U$-flow and modified $U$-flow approximations $\bar{\Sigma}^{\mathrm{PUF}}, \bar{\Sigma}^{\mathrm{MUF}}$ given by Eqs. (41) and (42); additionally, the static variant $\bar{\Sigma} \mathrm{StUF}$ from Eq. 43); finally, the $C U$-flow approximation $\bar{\Sigma}^{\mathrm{CUF}_{\Lambda}}$ from Eq. (51) [with $\Lambda$ referring to the definition of the cut-off in Eq. [530] and the $C$-flow approximation $\bar{\Sigma}^{\mathrm{CF}}$ according to Eq. (54) (which is not equivalent to self-consistent perturbation theory).

\section{APPLICATION TO THE ANDERSON IMPURITY MODEL}

\section{A. Hamiltonian and action}

The dot Hamiltonian of the single impurity Anderson model is

$$
\begin{aligned}
H_{\mathrm{dot}} & =\sum_{\sigma}\left(V_{\mathrm{g}}+\sigma B\right) d_{\sigma}^{\dagger} d_{\sigma}+U\left(d_{\uparrow}^{\dagger} d_{\uparrow}-\frac{1}{2}\right)\left(d_{\downarrow}^{\dagger} d_{\downarrow}-\frac{1}{2}\right) \\
& =\sum_{\sigma} \epsilon_{\sigma} d_{\sigma}^{\dagger} d_{\sigma}-\sum_{\sigma} \frac{U}{2} d_{\sigma}^{\dagger} d_{\sigma}+U d_{\uparrow}^{\dagger} d_{\uparrow} d_{\downarrow}^{\dagger} d_{\downarrow}+\text { const. }
\end{aligned}
$$

We combine the gate voltage $V_{\mathrm{g}}$ and the magnetic field $B$ in a single-particle energy $\epsilon_{\sigma}=V_{\mathrm{g}}+\sigma B$, with $\sigma= \pm 1=$ $\uparrow, \downarrow$. The interaction $U$ is introduced in a particle-hole symmetric way which entails an additional single-particle term as illustrated in the second line.

The dot is coupled to a semi-infinite lead of non-interacting fermions by a momentum- and spinindependent coupling $t$. We perform the wide-band limit, which means to assume a constant lead density of states on the whole energy axis. As a consequence, we can account for the lead by a constant hybridization $\Gamma=\pi \rho_{\text {lead }}(0)|t|^{2}$ in the free dot propagator, in which $\rho_{\text {lead }}(0)$ denotes the density of states at the end of the lead. The whole system is prepared in grand canonical equilibrium with temperature $T=1 / \beta$ and chemical potential $\mu=0$. For the numerical evaluation we choose $T=0$. The action entering the formula (1) for the partition function has the form

$$
\begin{aligned}
S[\psi]= & \frac{1}{2} \sum_{\alpha \alpha^{\prime}} \psi_{\alpha}\left(-C_{\alpha \alpha^{\prime}}^{-1}+U_{\alpha \alpha^{\prime}}^{(1)}\right) \psi_{\alpha^{\prime}} \\
& +\frac{1}{4 !} \sum_{\alpha_{1} \alpha_{2} \alpha_{3} \alpha_{4}} U_{\alpha_{1} \alpha_{2} \alpha_{3} \alpha_{4}}^{(2)} \psi_{\alpha_{1}} \psi_{\alpha_{2}} \psi_{\alpha_{3}} \psi_{\alpha_{4}} .
\end{aligned}
$$


Compared to Eq. (2) we have an additional quadratic contribution due to the interaction. This could as well be absorbed into the inverse free propagator. However, we prefer a quadratic contribution to the interaction, as it allows for a more transparent treatment of particlehole symmetry and for a clear distinction of $U$-flow and $C$-flow. As the leads are integrated out, the multi-indices $\alpha=\left(c, \sigma, \nu_{n}\right)$ contain no lead states but only dot states $\sigma$. Furthermore, we switch to Matsubara frequencies $\nu_{n}=$ $\frac{\pi}{\beta}(2 n+1)$ instead of imaginary times $\tau$. In the following we discuss the constituents of the action.

Free propagator and Fourier transform. In the usual Fourier transform without charge indices $\left(\int_{\tau}=\int_{0}^{\beta} d \tau\right)$

$$
\begin{aligned}
\left(C_{\mathrm{reg}}^{-1}\right)_{n_{1} n_{1}^{\prime}}^{\sigma_{1} \sigma_{1}^{\prime}} & =\int_{\tau_{1}} \int_{\tau_{1}^{\prime}} e^{i \nu_{n_{1}} \tau_{1}}\left(C^{-1}\right)^{\sigma_{1} \sigma_{1}^{\prime}}\left(\tau_{1}, \tau_{1}^{\prime}\right) e^{-i \nu_{n_{1}^{\prime}} \tau_{1}^{\prime}} \\
& =\beta \delta_{n_{1}, n_{1}^{\prime}} \delta_{\sigma_{1}, \sigma_{1}^{\prime}} C_{\mathrm{reg}, \sigma}^{-1}\left(\nu_{n}\right)
\end{aligned}
$$

the inverse lead-dressed free propagator on the dot reads as

$$
C_{\mathrm{reg}, \sigma}^{-1}\left(\nu_{n}\right)=i \nu_{n}-\epsilon_{\sigma}+i \operatorname{sgn}\left(\nu_{n}\right) \Gamma
$$

In this work based on the charge index notation, we use a different convention for the Fourier transform $[y=(\sigma, c)]$ :

$$
\begin{aligned}
G_{n_{1} n_{1}^{\prime}}^{y} y_{1}^{\prime} & =\int_{\tau_{1}} \int_{\tau_{1}^{\prime}} e^{-i \nu_{n_{1}} \tau_{1}} G^{y_{1} y_{1}^{\prime}}\left(\tau_{1}, \tau_{1}^{\prime}\right) e^{-i \nu_{n_{1}^{\prime}} \tau_{1}^{\prime}}, \\
\Sigma_{n_{1} n_{1}^{\prime}}^{y_{1} y_{1}^{\prime}} & =\int_{\tau_{1}} \int_{\tau_{1}^{\prime}} e^{i \nu_{n_{1}} \tau_{1}} \Sigma^{y_{1} y_{1}^{\prime}}\left(\tau_{1}, \tau_{1}^{\prime}\right) e^{i \nu_{n_{1}^{\prime}} \tau_{1}^{\prime}} .
\end{aligned}
$$

For the vertex-like $C^{-1}$, the two conventions are connected in the following way $(\bar{c}=-c)$ :

$$
\left(C^{-1}\right)_{\sigma \sigma^{\prime}, n n^{\prime}}^{c c^{\prime}}=c \delta_{c, \bar{c}^{\prime}} \delta_{\sigma, \sigma^{\prime}} \delta_{n+n^{\prime}, 0} C_{\mathrm{reg}, \sigma}^{-1}\left(\nu_{n}\right) .
$$

Here, $\delta_{n+n^{\prime}, 0}$ is a sloppy short-hand notation for $\delta_{\nu_{n}+\nu_{n^{\prime}}, 0}$, that is for the requirement $\frac{\pi}{\beta}\left(2 n+1+2 n^{\prime}+1\right)=$ 0 or $n+n^{\prime}+1=0$. From

$$
\left(C^{-1}\right)_{\sigma \sigma^{\prime}, n n^{\prime}}^{c c^{\prime}}=\beta \delta_{c, \bar{c}^{\prime}} \delta_{\sigma, \sigma^{\prime}} \delta_{n+n^{\prime}, 0}\left(C^{-1}\right)_{\sigma, n}^{c},
$$

we deduce

$$
\left(C^{-1}\right)_{\sigma, n}^{c}=c\left[i \nu_{n}-\epsilon_{\sigma}+i \operatorname{sgn}\left(\nu_{n}\right) \Gamma\right] .
$$

In order to derive a rule for inversion, let $A$ denote a self-energy or propagator. From

$$
\begin{aligned}
\sum_{\sigma^{\prime}, c^{\prime}} \int_{0}^{\beta} d \tau^{\prime}(A)_{\sigma \sigma^{\prime}}^{c c^{\prime}}\left(\tau, \tau^{\prime}\right) & \left(A^{-1}\right)_{\sigma^{\prime} \sigma^{\prime \prime}}^{c^{\prime} c^{\prime \prime}}\left(\tau^{\prime}, \tau^{\prime \prime}\right) \\
& =\delta\left(\tau-\tau^{\prime \prime}\right) \delta_{\sigma, \sigma^{\prime \prime}} \delta_{c, c^{\prime \prime}}
\end{aligned}
$$

in time-space follows

$$
\frac{1}{\beta} \sum_{n^{\prime}, \sigma^{\prime}, c^{\prime}}(A)_{\sigma \sigma^{\prime}, n n^{\prime}}^{c c^{\prime}}\left(A^{-1}\right)_{\sigma^{\prime} \sigma^{\prime \prime}, n^{\prime} n^{\prime \prime}}^{c^{\prime} c^{\prime \prime}}=\beta \delta_{n, n^{\prime \prime}} \delta_{\sigma, \sigma^{\prime \prime}} \delta_{c, c^{\prime \prime}}
$$

We thus find

$$
A_{\sigma, n}^{c}=\frac{1}{\left(A^{-1}\right)_{\sigma,-n}^{\bar{c}}} \text {. }
$$

For the Anderson model, the antisymmetry of two-point functions means $A_{\sigma \sigma^{\prime}, n n^{\prime}}^{c c^{\prime}}=-A_{\sigma^{\prime} \sigma, n^{\prime} n}^{c^{\prime} c}$. Due to $A^{c c^{\prime}} \sim$ $\delta_{c, \bar{c}^{\prime}}$, it is thus sufficient to use either the $c=+$ or the $c=-$ component. We choose to use $c=+$ for the selfenergy and $c=-$ for propagators, i.e.

$$
\begin{aligned}
& C_{\sigma, n}^{-}=\frac{1}{\left(C^{-1}\right)_{\sigma,-n}^{+}}=-\frac{1}{i \nu_{n}+\epsilon_{\sigma}+i \operatorname{sgn}\left(\nu_{n}\right) \Gamma}, \\
& G_{\sigma, n}^{-}=\frac{1}{\left(G^{-1}\right)_{\sigma,-n}^{+}}=\frac{1}{\left(C^{-1}\right)_{\sigma,-n}^{+}-\Sigma_{\sigma,-n}^{+}} .
\end{aligned}
$$

Interaction part of the action. The quadratic interaction contribution to the action is

$$
-\frac{U}{2} \int_{0}^{\beta} d \tau \sum_{\sigma} \bar{\psi}_{\sigma}(\tau) \psi_{\sigma}(\tau)=\frac{1}{2} \sum_{\alpha \alpha^{\prime}} \psi_{\alpha} U_{\alpha \alpha^{\prime}}^{(1)} \psi_{\alpha^{\prime}}
$$

with

$$
U_{\sigma \sigma^{\prime}, n n^{\prime}}^{(1), c c^{\prime}}=c \beta \delta_{n+n^{\prime}, 0} \delta_{\sigma \sigma^{\prime}} \delta_{c \bar{c}^{\prime}} \frac{U}{2}
$$

The quartic interaction contribution to the action is

$$
\begin{aligned}
& U \int_{0}^{\beta} d \tau \bar{\psi}_{\uparrow}(\tau) \bar{\psi}_{\downarrow}(\tau) \psi_{\downarrow}(\tau) \psi_{\uparrow}(\tau) \\
& \quad=\frac{1}{4 !} \sum_{\alpha_{1} \ldots \alpha_{4}} U_{\alpha_{1} \alpha_{2} \alpha_{3} \alpha_{4}}^{(2)} \psi_{\alpha_{1}} \psi_{\alpha_{2}} \psi_{\alpha_{3}} \psi_{\alpha_{4}}
\end{aligned}
$$

In order to determine $U^{(2)}$ we use that

$$
U d_{\uparrow}^{\dagger} d_{\uparrow} d_{\downarrow}^{\dagger} d_{\downarrow}=\frac{1}{2 !^{2}} \sum_{\sigma_{1} \sigma_{2} \sigma_{3} \sigma_{4}} U_{\sigma_{1} \sigma_{2} \sigma_{3} \sigma_{4}}^{-} d_{\sigma_{1}}^{\dagger} d_{\sigma_{2}}^{\dagger} d_{\sigma_{4}} d_{\sigma_{3}}
$$

with

$$
U_{\sigma_{1} \sigma_{2} \sigma_{3} \sigma_{4}}^{-}=\delta_{\sigma_{1}, \bar{\sigma}_{2}} \delta_{\sigma_{3}, \bar{\sigma}_{4}}\left[\delta_{\sigma_{1}, \sigma_{3}}-\delta_{\sigma_{1}, \bar{\sigma}_{3}}\right] U
$$

Hence,

$$
U_{\sigma_{1} \sigma_{2} \sigma_{3} \sigma_{4}, n_{1} n_{2} n_{3} n_{4}}^{(2) c_{1} c_{2} c_{3} c_{4}}=\beta \delta_{n_{1}+n_{2}+n_{3}+n_{4}, 0} U_{\sigma_{1} \sigma_{2} \sigma_{3} \sigma_{4}}^{(2) c_{1} c_{2} c_{3} c_{4}}
$$

with

$$
U_{\sigma_{1} \sigma_{2} \sigma_{3} \sigma_{4}}^{(2) c_{3} c_{2} c_{4}}=\left\{\begin{array}{cl}
-U_{\sigma_{1} \sigma_{2} \sigma_{3} \sigma_{4}}^{-} & \text {if } c_{1}=c_{2}=\bar{c}_{3}=\bar{c}_{4} \\
U_{\sigma_{1}}^{-} \sigma_{3} \sigma_{2} \sigma_{4} & \text { if } c_{1}=c_{3}=\bar{c}_{2}=\bar{c}_{4} \\
-U_{\sigma_{2} \sigma_{3} \sigma_{1} \sigma_{4}}^{-} & \text {if } c_{2}=c_{3}=\bar{c}_{1}=\bar{c}_{4} \\
0 & \text { else }
\end{array}\right.
$$

We note that the majority of the $2^{8}=256$ components in Eq. (76) are zero. 


\section{B. The quadratic interaction part in the self-energy equations and (un-)restricted MUF}

The quadratic interaction contribution to the action causes a few minor changes to the equations for the selfenergy, which we summarize now. First of all, we replace Eq. (6) for the definition of the Luttinger-Ward functional by

$\Phi[G]=\Gamma[G]-\frac{1}{2} \operatorname{tr} \ln (-G)+\frac{1}{2} \operatorname{tr}\left[\left(C^{-1}+U^{(1)}\right) G-1\right]$.

Then $\Phi$ is again minus the sum of all closed skeleton diagrams made of two-particle vertices $U^{(2)}$ and full propagator lines $G$. In particular, $\Phi$ does not depend on the one-particle vertex $U^{(1)}$. Equation (7) for the self-energy functional now reads as

$$
\Sigma[G]=-\Phi^{(1)}[G]-U^{(1)} .
$$

We induce the $U$-flow by a flow parameter in the twoparticle interaction, $U^{(2)} \rightarrow U_{\lambda}^{(2)}$. For all our $U$-flow schemes except MUF, particle-hole symmetry during all of the flow is ensured by dressing $U^{(1)}=U_{\lambda}^{(1)}$ with the corresponding $\lambda$-dependence. As $\Phi$ does not depend on $U^{(1)}$, the flow equations for $\Phi$ and $\Phi^{(1)}$ maintain the form derived in Sec. IV (now with the notation $U^{(2)}$ instead of $U$ for the two-particle vertex). However, the singleparticle vertex $U^{(1)}$ enters the self-energy $\Sigma=-\Phi^{(1)}-$ $U^{(1)}$. Consequently, an addend $-U^{(1)}$ must be added to the self-consistency equations (34) and (37) of cfRG and FLEX. For instance, Eq. 37) is replaced by

$$
\begin{aligned}
\Sigma^{\mathrm{FLEX}}=-U^{(2)} \cdot G & -\frac{4}{3}\left(U^{(2)} \cdot \Pi \cdot U^{(2)}\right)^{\mathrm{R}} \cdot G \\
& +2\left(\Upsilon \cdot U^{(2)}\right)^{\mathrm{R}} \cdot G-U^{(1)}
\end{aligned}
$$

Similarly, an addend $-\dot{U}_{\lambda}^{(1)}$ enters the flow equations 41, 43 and (51) for the PUF, StUF and CUF approximations. For instance, Eq. (41) is replaced by

$$
\begin{aligned}
\dot{\bar{\Sigma}}_{\lambda}^{\mathrm{PUF}}= & -\frac{2}{3}\left(\bar{\Upsilon}_{\lambda} \cdot \dot{U}_{\lambda}^{(2)} \cdot \bar{\Upsilon}_{\lambda}^{\mathrm{T}}-\bar{\Upsilon}_{\lambda} \cdot \dot{U}_{\lambda}^{(2)}-\dot{U}_{\lambda}^{(2)} \cdot \bar{\Upsilon}_{\lambda}^{\mathrm{T}}\right)^{\mathrm{R}} \cdot \bar{G}^{\lambda} \\
& -\dot{U}_{\lambda}^{(2)} \cdot \bar{G}_{\lambda}-U_{\lambda}^{(2)} \cdot \dot{\bar{G}}_{\lambda}-\dot{U}_{\lambda}^{(1)} .
\end{aligned}
$$

The initial conditions for these three flow schemes remain unchanged because $U_{\lambda_{i}}^{(1)}=0$.

For the MUF approximation, we leave $U^{(1)}$ independent of $\lambda$ to ensure particle-hole symmetry. The flow equation 42 remains unchanged. However, the self-consistent Hartree-Fock initial condition now reads $\bar{\Sigma}_{\lambda_{\mathrm{i}}}=-U^{(2)} \cdot \bar{G}_{\lambda_{\mathrm{i}}}-U^{(1)}$. For the Anderson model, the self-consistent Hartree-Fock method predicts an unphysical spin-symmetry breaking for $U>U_{\text {crit }}=\pi \Gamma$ (at $\left.V_{\mathrm{g}}=0=B\right)$ : there are two "unrestricted" magnetic solutions which can be mapped onto one another by flipping the spins. There is yet another, "restricted", solution which is non-magnetic but responds unphysically to infinitesimal magnetic fields, having a negative spin susceptibility. We can choose any of these solutions as starting point of the modified $U$-flow. Accordingly, we obtain two different MUF schemes for $U>U_{\text {crit }}$ which we call "restricted MUF" and "unrestricted MUF". The question arises as to whether the flow is able to eliminate the artifacts introduced by the initial conditions. The numerical results described in Sec. VID show that this is not the case.

For the CF approximation, neither $U^{(2)}$ nor $U^{(1)}$ is made $\lambda$-dependent and the flow equation (54) stays the same. However, the numerical initial condition (55) is changed to

$$
\bar{\Sigma}_{\lambda_{\mathrm{i}}^{\text {num }}}^{\mathrm{CF}}=\lim _{\lambda_{0} \rightarrow \infty} \lim _{\eta \rightarrow 0^{+}} \int_{\infty}^{\lambda_{0}} d \lambda\left(-U^{(2)} \cdot \bar{G}_{\lambda}\right)-U^{(1)} .
$$

\section{Steps towards implementable equations for the Anderson impurity model}

In Appendix A, we derive specifically for the Anderson model the relevant equations for the numerical computation of the self-energy. Here, we summarize the important steps.

In Appendix A 1, a suitable reduced index notation is defined. It exploits that the number of indices on fourpoint functions can be reduced significantly by making use of symmetry relations. Furthermore, many components can be shown to be zero due to particle-number and spin conservation.

In Appendix A 2 it is shown how to calculate $\bar{\Upsilon}$. While the four-point function $\bar{\Upsilon}$ depends on four frequencies or rather on three independent frequencies, we find that one frequency is always summed over independently. We thus define a $\widetilde{\Upsilon}$ which depends only on the two remaining frequencies. $\widetilde{\Upsilon}$ then turns out to depend only on the sum of the two frequencies which is only one composite (bosonic) frequency. The non-zero components of $\widetilde{\Upsilon}$ are identified with a particular channel (particle-particle, direct or exchange particle-hole) and are labeled accordingly.

In Appendix A 3, the self-consistency equations for the self-energy of cfRG and FLEX are cast into a form suitable for numerical implementation. When we evaluate the dot products in the self-consistency equations (34) and (37) (adapted according to Sec. VB), we exploit the sparseness of the components mentioned in the preceding paragraphs. We then perform the $T=0$ limit. The final resulting equation is

$$
\begin{aligned}
\bar{\Sigma}_{\sigma}(\nu)= & U \int_{0}^{\infty} \frac{d \nu^{\prime}}{\pi} \operatorname{Re}\left[\bar{G}_{\bar{\sigma}}\left(\nu^{\prime}\right)\right] \\
& +U \int_{-\infty}^{\infty} \frac{d \omega}{2 \pi}\left\{\kappa_{\mathrm{p}}\left[\kappa_{0} \widetilde{\Psi}^{\mathrm{p}}(\omega)-\widetilde{\Upsilon}^{\mathrm{p}}(\omega)\right] \bar{G}_{\bar{\sigma}}(\nu-\omega)\right. \\
& +\kappa_{\mathrm{d}}\left[\widetilde{\Upsilon}^{\mathrm{d} \sigma}(\omega)-\kappa_{0} \widetilde{\Psi}^{\mathrm{d} \sigma}(\omega)\right] \bar{G}_{\sigma}(\omega-\nu) \\
& \left.+\kappa_{\mathrm{x}}\left[\kappa_{0} \widetilde{\Psi}^{\mathrm{x} \sigma}(\omega)-\widetilde{\Upsilon}^{\mathrm{x} \sigma}(\omega)\right] \bar{G}_{\bar{\sigma}}(\omega-\nu)\right\}
\end{aligned}
$$


It describes either FLEX or cfRG, depending on the choice of the newly introduced coefficients $\kappa_{i}, i=$ $0, p, d, x$. We solve the equation numerically by iteration. The details of the numerical implementation, e.g. the use of frequency grids, are discussed in Appendix B. We take zero as the initial guess for the iteration of the self-energy. If we plainly iterated over Eq. (82), the value of $U$ would be limited by the critical value $U_{\text {crit }}=\pi \Gamma$ known from the self-consistent Hartree-Fock solution. $\frac{38}{}$ In order to circumvent this problem, we gradually increase $U$ in each step of the iteration up to the desired value; this idea was already applied in Ref. 38, In addition, we calculate the next guess of an iteration step by combining the last guess and the outcome of the selfconsistency equation in a weighted manner. We found empirically that this makes the iteration faster and more stable.

In App. A4, we turn to the flow equations for the selfenergy for the various non-conserving methods. One proceeds as for the conserving case and obtains

$$
\begin{aligned}
\dot{\bar{\Sigma}}_{\sigma, \lambda}^{\mathrm{PUF}}(\nu)= & \frac{\dot{U}_{\lambda}}{3} \int_{-\infty}^{\infty} \frac{d \omega}{2 \pi}\left\{\left[\widetilde{\Upsilon}_{\lambda}^{\mathrm{p}}(\omega)^{2}-2 \widetilde{\Upsilon}_{\lambda}^{\mathrm{p}}(\omega)\right] \bar{G}_{\bar{\sigma}}^{\lambda}(\nu-\omega)\right. \\
& +2 \widetilde{\Upsilon}_{\lambda}^{\mathrm{d} \sigma}(-\omega)\left[1-\widetilde{\Upsilon}_{\lambda}^{\overline{\mathrm{d}}}(\omega)\right] \bar{G}_{\sigma}^{\lambda}(\omega-\nu) \\
& \left.+\left[\widetilde{\Upsilon}_{\lambda}^{\mathrm{x} \sigma}(\omega)^{2}-2 \widetilde{\Upsilon}_{\lambda}^{\mathrm{x} \sigma}(\omega)\right] \bar{G}_{\bar{\sigma}}^{\lambda}(\omega-\nu)\right\} \\
& +\dot{U}_{\lambda} \int_{-\infty}^{\infty} \frac{d \nu^{\prime}}{\pi} \operatorname{Re}\left[\bar{G}_{\bar{\sigma}}^{\lambda}\left(\nu^{\prime}\right)\right] \\
& +U_{\lambda} \int_{-\infty}^{\infty} \frac{d \nu^{\prime}}{2 \pi} \dot{\bar{G}}_{\bar{\sigma}}^{\lambda}\left(\nu^{\prime}\right)
\end{aligned}
$$

for the PUF,

$$
\begin{aligned}
\dot{\bar{\Sigma}}_{\sigma, \lambda}^{\mathrm{MUF}}(\nu)= & \frac{\dot{U}_{\lambda}}{3} \int_{-\infty}^{\infty} \frac{d \omega}{2 \pi}\left\{\left[\widetilde{\Upsilon}_{\lambda}^{\mathrm{p}}(\omega)^{2}-2 \widetilde{\Upsilon}_{\lambda}^{\mathrm{p}}(\omega)\right] \bar{G}_{\bar{\sigma}}^{\lambda}(\nu-\omega)\right. \\
& +2 \widetilde{\Upsilon}_{\lambda}^{\mathrm{d} \sigma}(-\omega)\left[1-\widetilde{\Upsilon}_{\lambda}^{\mathrm{d}}(\omega)\right] \bar{G}_{\sigma}^{\lambda}(\omega-\nu) \\
& \left.+\left[\widetilde{\Upsilon}_{\lambda}^{\mathrm{x} \sigma}(\omega)^{2}-2 \widetilde{\Upsilon}_{\lambda}^{\mathrm{x} \sigma}(\omega)\right] \bar{G}_{\bar{\sigma}}^{\lambda}(\omega-\nu)\right\} \\
& +U \int_{-\infty}^{\infty} \frac{d \nu^{\prime}}{2 \pi} \dot{\bar{G}}_{\bar{\sigma}}^{\lambda}\left(\nu^{\prime}\right)
\end{aligned}
$$

for the MUF,

$$
\dot{\bar{\Sigma}}_{\sigma}^{\mathrm{CUF}_{\Lambda}}(\nu)=\dot{\bar{\Sigma}}_{\sigma}^{\mathrm{PUF}}(\nu)+\Delta_{\sigma}^{\lambda}(\nu)
$$

for the CUF and

$$
\dot{\bar{\Sigma}}_{\sigma}^{\mathrm{CF}}(\nu)=U \int_{-\infty}^{\infty} \frac{d \nu^{\prime}}{2 \pi} \dot{\bar{G}}_{\bar{\sigma}}^{\lambda}\left(\nu^{\prime}\right)+\left.\Delta_{\sigma}^{\lambda}(\nu)\right|_{U_{\lambda} \rightarrow U}
$$

for the CF approximation. In case of the StUF approximation, the frequency integral on the right-hand side can be evaluated analytically and one finds the compact equation

$$
\dot{\bar{\Sigma}}_{\sigma, \lambda}^{\mathrm{StUF}}=-\frac{\dot{U}_{\lambda}}{\pi} \operatorname{atan}\left(\frac{\epsilon_{\bar{\sigma}}+\bar{\Sigma}_{\bar{\sigma}, \lambda}^{\mathrm{StUF}}}{\Gamma}\right) .
$$

In our numerics, we evolve the self-energy according to the respective flow equation by a standard differential equation solver. For the frequency dependent schemes we use the same frequency grid as in the conserving case. For more details on the implementation, see also Appendix B.

In all methods (except for StUF), the right-hand side contains an integral over a bosonic frequency which must be carried out numerically. This must be done for each fermionic frequency of the self-energy in each step of the iteration or flow. As the fermionic grid is given by $n_{\text {len }}$ frequencies and the bosonic grid by $m_{\text {len }}=2 n_{\text {len }}$ frequencies (cf. Appendix B), the effort of the methods scales as $\mathcal{O}\left(n_{\text {len }}^{2}\right)$ in each step. The same scaling behavior is known from a $1 \mathrm{PI}$ vertex expansion Matsubara fRG applied to the Anderson model which uses a flowing frequency dependent two-particle vertex in channel decomposition. 30

\section{The computation of observables}

For both, conserving and non-conserving methods, we use the numerical solution $\bar{\Sigma}_{\sigma}(\nu)$ to compute observables. The occupancy can be obtained from the propagator according to Eq. (15) via

$$
\left\langle n_{\sigma}\right\rangle_{\text {prop }}=\int_{-\infty}^{\infty} \frac{d \nu}{2 \pi} \bar{G}_{\sigma}(\nu) e^{-i \nu 0^{+}}=\frac{1}{2}+\int_{0}^{\infty} \frac{d \nu}{\pi} \operatorname{Re}\left[\bar{G}_{\sigma}(\nu)\right] .
$$

Even though we investigate an equilibrium setup in which we couple the dot to one lead by $\Gamma$, we can calculate the (linear-response) conductance which a system coupled to two leads by $\Gamma / 2$ would have at zero bias voltage ${ }^{40}$ The conductance is given by

$$
G^{\text {cond }}=\frac{e^{2}}{h} \Gamma \sum_{\sigma} \operatorname{Im}\left[\bar{G}_{\sigma}\left(0^{+}\right)\right]
$$

with $h=2 \pi \hbar=2 \pi$. At $T=B=V_{\mathrm{g}}=0$, the so-called effective mass is defined via

$$
m^{*}=1-\lim _{\nu \searrow 0} \frac{d \operatorname{Im} \bar{\Sigma}_{\sigma}(\nu)}{d \nu}
$$

which is independent of $\sigma$ due to $B=0$. We are also interested in the static spin and charge susceptibility given by the derivatives

$$
\begin{gathered}
\chi_{\mathrm{s}}=-\left.\frac{d\left\langle n_{\uparrow}-n_{\downarrow}\right\rangle_{\text {prop }}}{d B}\right|_{B=0}, \\
\chi_{\mathrm{c}}=-\left.\frac{d\left\langle n_{\uparrow}+n_{\downarrow}\right\rangle_{\text {prop }}}{d V_{\mathrm{g}}}\right|_{V_{\mathrm{g}}=0} .
\end{gathered}
$$

Numerically, we probe by a very small magnetic field $\left(B / \Gamma=10^{-5}\right)$ or a shift of the gate voltage $\left(V_{\mathrm{g}} / \Gamma=\right.$ 
$\left.10^{-4}\right)$ and compute the finite difference approximations

$$
\begin{gathered}
\chi_{\mathrm{s}} \approx \frac{\left\langle n_{\downarrow}-n_{\uparrow}\right\rangle_{\text {prop }}-\left\langle n_{\downarrow}-n_{\uparrow}\right\rangle_{\text {prop }} \mid B=0}{B}, \\
\chi_{\mathrm{c}} \approx-\frac{\left\langle n_{\downarrow}+n_{\uparrow}\right\rangle_{\text {prop }}-\left\langle n_{\downarrow}+n_{\uparrow}\right\rangle_{\text {prop }} \mid V_{\mathrm{g}}=0}{V_{\mathrm{g}}} .
\end{gathered}
$$

\section{E. Alternative approaches to the occupancy as test for conserving approximations}

In Sec. III we explained that $\Phi$-derivable approximations (such as FLEX and cfRG) are thermodynamically consistent and preserve the Friedel sum rule. This assures coinciding results when the impurity occupancy is computed either from the propagator [Eq. (88)] or from the grand potential [Eq. (16)] or from the Friedel sum rule. The latter reads as

$$
\left\langle n_{\sigma}\right\rangle_{\mathrm{FSR}}=\frac{1}{2}-\frac{1}{\pi} \operatorname{atan}\left(\frac{\epsilon_{\sigma}+\operatorname{Re}\left[\bar{\Sigma}_{\sigma}\left(0^{+}\right)\right]}{\Gamma}\right)
$$

for the Anderson model at zero temperature and in the wide band limit. ${ }^{40}$ Based on Sec. IV we expect the PUF, StUF and MUF schemes to be non- $\Phi$-derivable methods. In the results Sec. VIC, we will indeed see that for these methods the three ways to the occupancy lead to disagreeing results.

Let us describe in more detail how we evaluate the occupancy from the grand potential. From Eq. (16) follows that

$$
\left\langle n_{\uparrow}+n_{\downarrow}\right\rangle_{\mathrm{gp}}=\frac{d}{d V_{\mathrm{g}}} \bar{\Omega} .
$$

For the $\Phi$-derivable schemes, we obtain $\bar{\Omega}$ as the sum of $\left.\bar{\Omega}\right|_{U=0}$ and $\Delta \bar{\Omega}=\bar{\Omega}-\left.\bar{\Omega}\right|_{U=0}$ which we can calculate directly from $\bar{\Sigma}$. This yields

$$
\begin{aligned}
\left\langle n_{\uparrow}+n_{\downarrow}\right\rangle_{\mathrm{gp}} & =\frac{d}{d V_{\mathrm{g}}}\left(\Delta \bar{\Omega}+\left.\bar{\Omega}\right|_{U=0}\right) \\
& =\frac{d}{d V_{\mathrm{g}}} \Delta \bar{\Omega}+\left\langle n_{\uparrow}+n_{\downarrow}\right\rangle_{U=0} .
\end{aligned}
$$

The non-interacting occupancy is given by

$$
\left\langle n_{\uparrow}+n_{\downarrow}\right\rangle_{U=0}=\sum_{\sigma}\left[\frac{1}{2}-\frac{1}{\pi} \operatorname{atan}\left(\frac{\epsilon_{\sigma}}{\Gamma}\right)\right] .
$$

For the flow schemes, $\bar{\Omega}=\bar{\Omega}_{\lambda_{\mathrm{f}}}$ leads to

$$
\begin{aligned}
\left\langle n_{\uparrow}+n_{\downarrow}\right\rangle_{\mathrm{gp}} & =\frac{d}{d V_{\mathrm{g}}} \bar{\Omega}_{\lambda_{\mathrm{f}}}=\frac{d}{d V_{\mathrm{g}}} \int_{\lambda_{\mathrm{i}}}^{\lambda_{\mathrm{f}}} d \lambda \dot{\bar{\Omega}}_{\lambda}+\frac{d}{d V_{\mathrm{g}}} \bar{\Omega}_{\lambda_{\mathrm{i}}} \\
& =\frac{d}{d V_{\mathrm{g}}} \int_{\lambda_{\mathrm{i}}}^{\lambda_{\mathrm{f}}} d \lambda \dot{\bar{\Omega}}_{\lambda}+\left\langle n_{\uparrow}+n_{\downarrow}\right\rangle_{\lambda_{\mathrm{i}}} .
\end{aligned}
$$

Here, the second addend refers to

$$
\left\langle n_{\uparrow}+n_{\downarrow}\right\rangle_{\lambda_{\mathrm{i}}}=\sum_{\sigma}\left[\frac{1}{2}-\frac{1}{\pi} \operatorname{atan}\left(\frac{\epsilon_{\sigma}+\bar{\Sigma}_{\sigma, \lambda_{\mathrm{i}}}}{\Gamma}\right)\right]
$$

in which we exploit that for all schemes $\bar{\Sigma}_{\sigma, \lambda_{\mathrm{i}}} \in \mathbb{R}$ is frequency independent. The expressions for $\Delta \bar{\Omega}$ and $\dot{\bar{\Omega}}$ are provided in Appendix C. Numerically, the derivative with respect to the gate voltage is carried out by an interpolation routine.

\section{NUMERICAL RESULTS}

For the numerical investigations, we resort, as mentioned above, to the $T=0$ limit. The parameters for the frequency grids (see Appendix $\mathrm{B}$ ) are $n_{\mathrm{len}}=120$, $d \nu=10^{-6} \Gamma, \nu_{\max }=10^{8} \Gamma$. At selected values of the model parameters, we checked that this choice is sufficient to reach numerical convergence on the scale of the plots.

\section{A. Results for the conserving schemes}

In this section, we discuss the numerical results for each observable obtained with the cfRG approximation and with FLEX. The plots also show the PUF curves for comparison. These will be compared to the MUF results in Sec. VID. Figure 2 shows the effective mass, the charge and spin susceptibility as function of $U$, as well as the conductance as function of the gate voltage. We have chosen the parameters such that we can compare with published data. $.30|38| 41$ For this purpose, some plots include data obtained with $1 P I$ vertex expansion Matsubara fRG which takes into account at least a static flow of the 1PI two-particle vertex. For the effective mass and the spin susceptibility, we compare to more elaborate schemes which take into account the frequency dependence of the vertex (in its full or in a channel-decomposed form). Furthermore, we compare to numerically exact data from the numerical RG (NRG) or to exact Bethe ansatz results. The Bethe ansatz data were calculated via the formulas indicated in Ref. 42 or in case of the conductance taken from Ref. 43 and in case of the occupancy taken from Ref. 30 .

Our FLEX data coincide with the FLEX data presented by White in Ref. 38, 48 This confirms that the FLEX data are correctly determined, in particular as our implementation differs from that of White. White transformed the frequency integrations to the real axis while we work entirely on the imaginary axis.

cfRG, PUF and FLEX correctly describe the observables at very small $U$. The reason is that they are exact up to order $U^{2}$. However, when $U$ is increased, they deviate much earlier from the NRG or Bethe ansatz results than 1PI fRG. The cfRG approximation performs slightly better than FLEX and PUF. Generally, the results of all three approximations are similar. This is plausible since we found in Sec. IVB that cfRG and FLEX are closely related, and since we identified PUF in Sec. IV C as an approximation to cfRG. 

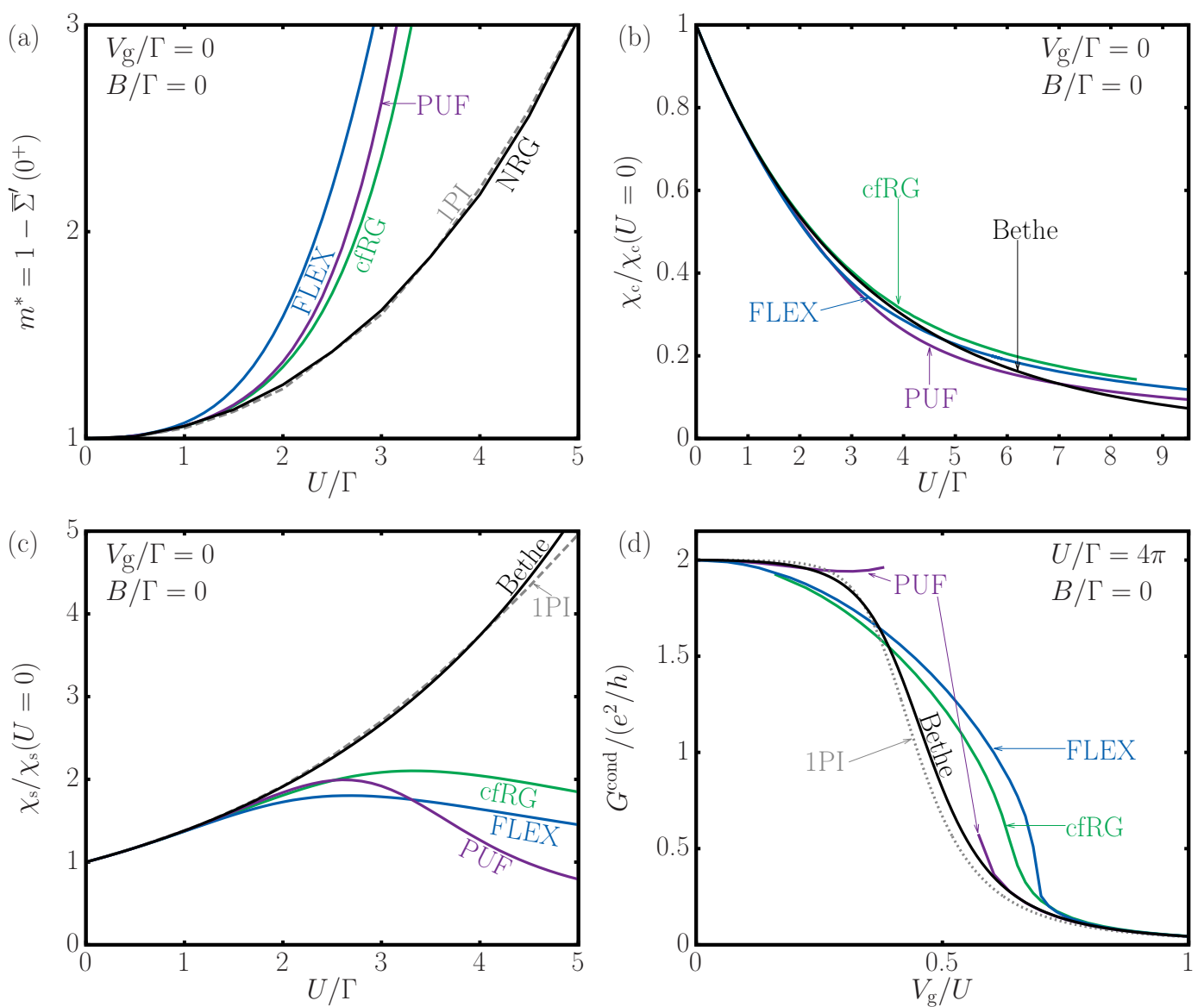

FIG. 2: (Color online) Numerical data for the cfRG, PUF and FLEX approximations. (a) Results for the effective mass are compared to 1PI fRG and NRG results from Fig. 6(a) in Ref. 30. (b) Results for the charge susceptibility are compared to Bethe ansatz results. We found well-converged solutions of the cfRG self-consistency equation only for $U<8.5 \Gamma$. (c) Results for the spin susceptibility are compared to 1PI fRG data from Fig. 6(b) in Ref. 30 and to Bethe ansatz results. (d) Results for the conductance as function of the gate voltage are compared to 1PI fRG data from Fig. 3 in Ref. 41 and Bethe ansatz data from Ref. 43. We found well-converged solutions of the cfRG self-consistency equation only for $V_{\mathrm{g}} \geq 2 \Gamma$.

Let us now discuss each plot in more detail. Concerning the effective mass shown in Fig. 2(a), cfRG, PUF and FLEX quickly overestimate the correct value. The FLEX data are reasonably precise up to $U \approx \Gamma$, those of cfRG and PUF up to $U \approx 1.5 \Gamma$. (For comparison, the shown 1PI fRG which employs channel decomposition provides good results up to $U \approx 5.5 \Gamma \stackrel{[30}{=}$ In Sec. VIB, we study a possible exponential behavior of the approximate effective mass.

We now turn to the charge susceptibility in Fig. 2(b). The FLEX and PUF data turn out to be trustworthy up to $U \approx 2 \Gamma$, those of $\operatorname{cfRG}$ up to $U \approx 3 \Gamma$. The FLEX and PUF curves lie below the Bethe ansatz curve at low $U$ and cross it as $U$ increases, while the cfRG curve always lies above. Nevertheless, all three approximations are roughly similar.

Let us proceed to the spin susceptibility in Fig. 2(c). Here, visible deviations of the FLEX data from the Bethe ansatz result start at $U \approx 1.5 \Gamma$; cfRG and PUF deviate only slightly later. All three approximations have in common that they produce values that are far too low for larger interaction strengths. They even show decreasing values instead of an exponential growth.

In Fig. 2(d), the conductance is shown. The cfRG and the FLEX data are again quite similar. Around zero gate voltage, they do not yield a conductance plateau, but instead a wide curved region. The PUF curve is remarkably distinct, with an overpronounced plateau and convergence problems around the plateau edge. This exceptional behavior is to be attributed to the large value of the interaction $U / \Gamma=4 \pi$ and it is lifted when turning to smaller values of $U / \Gamma$. We chose this large value in order to compare to existing data. Only at such large values, the conductance plateau is clearly visible.

\section{B. Hamann's prediction not confirmed}

In Ref. 22, Hamann investigates analytically an approximation to the self-energy of the Anderson model 

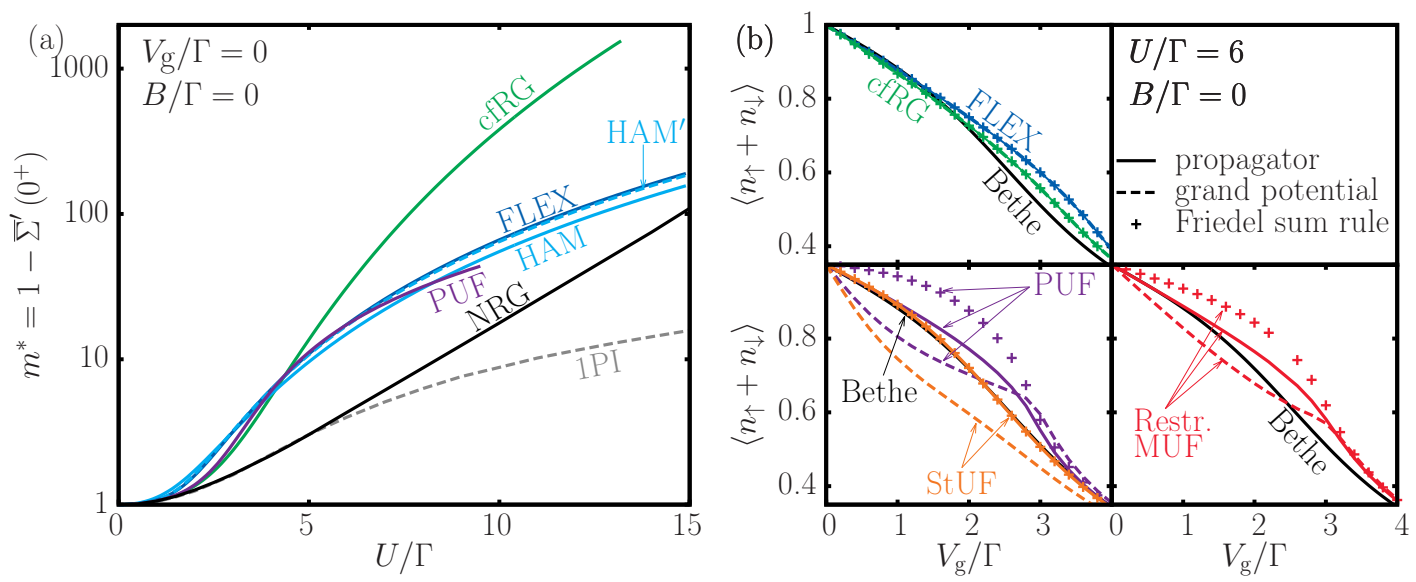

FIG. 3: (Color online) (a) The same numerical data for the effective mass as in Fig. 2(a), but on a logarithmic scale and including higher interaction strengths. The plot shows additionally data obtained with Hamann's approximation. (b) Numerical data for the occupancy of the dot, calculated for certain $\Phi$-derivable and non- $\Phi$-derivable schemes in various ways. The Bethe ansatz result (from Fig. 5 in Ref. $\underline{30 \text { ) }}$ is plotted for comparison.

which can be considered an ancestor of the FLEX method. For this approximation, which he attributes to Suh[ ${ }^{44}$, he predicts the occurrence of a characteristic temperature $\sim \exp \left[-\frac{1}{3}\left(\frac{U}{\pi \Gamma}\right)^{2}\right]$, as opposed to the exact Kondo temperature $\sim \exp \left(-\frac{\pi}{8} \frac{U}{\Gamma}\right)$. As the approximation is similar to FLEX, the characteristic temperature with quadratic exponent might as well appear for FLEX and the related cfRG and PUF. In this case the approximate effective mass should be proportional to $\exp \left[\frac{1}{3}\left(\frac{U}{\pi \Gamma}\right)^{2}\right]$. Here we show that the numerical data do not confirm this expectation, neither for FLEX and the fRG schemes, nor for Hamann's approximation itself.

Hamann's approximation ${ }^{22}$ to the self-energy can be derived from an approximate Luttinger-Ward functional with a diagrammatic representation almost identical to that of FLEX in Fig. 1. The difference is that the diagrams with particle-particle ladders are neglected and that the sum of diagrams with bubble chains (direct particle-hole channel) is approximated by $\frac{1}{2}$ the sum of diagrams with particle-hole ladders (exchange particlehole channel). Effectively, only the particle-hole ladder contribution is used, multiplied by a factor of $\frac{3}{2}$ for all diagrams from second order on. This yields a conserving approximation for the self-energy which does not capture second order perturbation theory with bare lines as the skeleton second order diagram is multiplied by $\frac{3}{2}$. By setting $\kappa_{0}=0, \kappa_{\mathrm{x}}=\frac{3}{2}$ and $\kappa_{\mathrm{p}}=\kappa_{\mathrm{d}}=0$ in Eq. 82 , we can calculate data according to this approach. We refer to this scheme by the index "HAM". A variant of Hamann's idea that takes into account the natural structure of Eq. A27) for FLEX is to set $\kappa_{0}=\frac{2}{3}, \kappa_{\mathrm{x}}=\frac{3}{2}$ and $\kappa_{\mathrm{p}}=\kappa_{\mathrm{d}}=0$. We thus define an alternative scheme "HAM" " according to this choice (which also does not capture second order perturbation theory correctly).

Figure 3 (a) presents again the effective mass data from Fig. 2(a), but on a logarithmic scale and up to larger val- ues of $U / \Gamma$, now including $\mathrm{HAM}$ and $\mathrm{HAM}^{\prime}$ data. We observe that the curves for FLEX and for HAM behave similarly. This confirms that Hamann's approach to replace all three FLEX channels by $3 / 2$ the particle-hole ladder is reasonable. We observe even better agreement (almost coincidence on the scale of the plot) of the alternative proposal $\mathrm{HAM}^{\prime}$ with FLEX. For large $U / \Gamma$, the NRG effective mass follows the exact result $\sim \exp \left(\frac{\pi}{8} \frac{U}{\Gamma}\right)$ which occurs as a straight line in the log-linear plot. According to Hamann's prediction, the curve corresponding to his approximation should increase quadratically in the loglinear plot at high $U / \Gamma$. This is obviously not the case; also the FLEX and the fRG (and HAM ${ }^{\prime}$ ) curves do not show this behavior. On the contrary, based on the data we expect that the NRG effective mass even surpasses the Hamann and the FLEX one from about $U \approx 18 \Gamma$ on. The reason for this discrepancy to Hamann's prediction remains to be clarified.

\section{Establishing that PUF, StUF and MUF are non- $\Phi$-derivable}

In this section we present numerical results which illustrate that the PUF, StUF and MUF schemes are non$\Phi$-derivable approximations. Fig. 3(b) shows the occupancy of the dot calculated for each scheme by the three ways suggested in Sec. $\mathrm{VE}$ from the propagator, from the grand potential and from the Friedel sum rule. For the cfRG and the FLEX method, the three ways correctly produce coinciding results, as expected for $\Phi$-derivable schemes. In contrast, each way produces a distinctly different result for the PUF, StUF and MUF schemes. As a single exception, the Friedel sum rule and integration of the propagator lead to coinciding results for the StUF approximation. This, however, is true for all static methods; for these, the propagator can be integrated analyti- 
cally to yield the Friedel sum rule. We have thus provided strong numerical evidence that PUF, StUF and MUF are indeed non- $\Phi$-derivable approximations. We remark that the same quantities were used to illustrate that truncated 1PI fRG is not thermodynamically consistent, cf. Fig. 5 of Ref. 30.

\section{Results for PUF and MUF}

In this section, we discuss the numerical results for the PUF and the MUF approximation. Figure 4 shows the same observables as above for these schemes.

The PUF and MUF results agree in acceptable limits with the exact ones only up to rather small $U / \Gamma \approx 1 \ldots 2$. This becomes particularly evident in the effective mass and spin susceptibility. Both, PUF and MUF, quickly overestimate the effective mass. Similarly, both quickly underestimate the spin susceptibility. These deviations are reminiscent of the FLEX results [compare to Fig.2(a) and 2 (c) or to Ref. 38. In fact, this similarity to FLEX extends to the charge susceptibility [compare to Figs. 2(b) or to Ref. 38].

A poor performance of the PUF scheme at larger interactions is already known from the quantum anharmonic oscillator which was studied in Ref. 20 as a toy model for quantum many-body systems. In contrast, the MUF approximation performs very well for the anharmonic oscillator. We attribute the poor performance for the Anderson model to the following reason: The success or failure of the MUF approximation is closely related to the success or failure of the self-consistent Hartree-Fock solution which is used as the starting point of the flow. This was already anticipated in Ref. 20, The HartreeFock method performs well for the anharmonic oscillator (within 3\% relative error compared to the exact result for a large range of interaction strengths). For the Anderson model in contrast, the Hartree-Fock solution is significantly less accurate. This explains the setback.

For $U>U_{\text {crit }}=\pi \Gamma$ the unrestricted Hartree-Fock solution as starting point of unrestricted MUF unphysically breaks the spin symmetry. The numerics indicate that the flow does not restore the symmetry; on the contrary, it even suffers from convergence problems. For the effective mass, the charge susceptibility and the spin susceptibility, the flow of unrestricted MUF does not come to end for $U \approx U_{\text {crit }} \ldots 8 \Gamma$. Furthermore, the values calculated for $U / \Gamma>8$ are not trustworthy. For the effective mass, they are unconvincingly high (not plotted); for the spin susceptibility, they are unstable and vary over a large range including negative values (not plotted); for the charge susceptibility, they are in an acceptable range but the method predicts a curvature around $U / \Gamma=8$ contradictory to all other schemes [see Fig. 4(b)]. Furthermore, the unrestricted MUF approximation does not reproduce the correct unitary conductance $G^{\text {cond }}=2 \frac{e^{2}}{h}$ at $V_{\mathrm{g}}=0$. This deficiency is shared by the unrestricted Hartree-Fock method [see Fig. 4(d)] and is obviously not settled by the flow.

These numerical findings of our unrestricted MUF scheme do not comply with a prediction made in Sec. IV.B of Ref. 19, There, it is argued that in 2PI fRG flow schemes a spurious symmetry breaking should decrease and eventually vanish during the flow due to the influence of Goldstone modes. This prediction derives from an analysis of the contribution $-\overline{\dot{\Phi}}_{\lambda}^{(1)}$ to the flow of the self-energy $\dot{\bar{\Sigma}}_{\lambda}=-\overline{\dot{\Phi}}_{\lambda}^{(1)}-\bar{\Phi}_{\lambda}^{(2)} \cdot \dot{\bar{G}}_{\lambda}$. This contribution is argued to reduce the symmetry breaking with increasing efficiency during the course of the RG flow.

It can be understood in more detail why the symmetry is not restored in our scheme. For $U$ moderately greater than $\pi \Gamma$, we can observe numerically that the symmetry breaking indeed starts to decrease during the flow. However, a divergence occurs in the flow equation before the symmetry is restored. One can understand analytically that this divergence necessarily occurs in our truncation scheme. The factor which diverges becomes apparent when the flow equation for the self-energy is formulated as non-self-consistent equation, $\dot{\bar{\Sigma}}_{\lambda}=-\left(I+\bar{\Phi}_{\lambda}^{(2)} \cdot \bar{\Pi}_{\lambda}\right)^{\text {inv }} \cdot \overline{\dot{\Phi}}_{\lambda}^{(1)}$, compare Eq. (98) of Ref. 20. In the MUF truncation, the factor $\left(I+\bar{\Phi}_{\lambda}^{(2)} \cdot \bar{\Pi}_{\lambda}\right)^{\text {inv }}$ takes the form $\left(I+U \cdot \bar{\Pi}_{\lambda}\right)^{\text {inv }}$ with bare interaction $U>\pi \Gamma$. This RPA-like series reaches a pole when the symmetry breaking becomes smaller; crucially, this happens before the symmetry is restored, since $U$ is greater than the critical value $U_{\text {crit }}=\pi \Gamma$ of the nonsymmetry-broken state. A more detailed analysis of this divergence is given in Appendix $\mathrm{B}$

For $U$ distinctly greater than $\pi \Gamma$, the flow of the selfenergy in the MUF truncation becomes more complicated. The emerging frequency dependence and imaginary parts of the self-energy then play a dominating role and the dressed RPA-like series no longer has a pole. Therefore, the MUF converges again from $U \approx 8 \Gamma$ on. However, only at the beginning of the flow the self-energy is essentially static and we observe numerically a tendency to suppress the symmetry breaking. In contrast, at the end of the flow the strongly frequency dependent self-energies for spin up and down differ largely.

We thus find the prediction of Ref. 19 that a spurious symmetry breaking vanishes automatically during the flow not fulfilled in our MUF scheme. The prediction of Ref. 19 might still be applicable to more advanced truncation schemes than our MUF.

Let us now turn to the restricted MUF. For the effective mass and the charge susceptibility, it is able to produce reasonable results above $U_{\text {crit }}$ that are comparable to those of PUF. For the spin susceptibility, in contrast, the flow does not come to an end for $U>U_{\text {crit }}$. This indicates that the unphysical response of the restricted Hartree Fock starting point to magnetic fields is not overcome by the RG flow. This is studied in more detail in Appendix B. For the conductance, the restricted MUF approximation predicts the correct value at $V_{\mathrm{g}}=0$. 

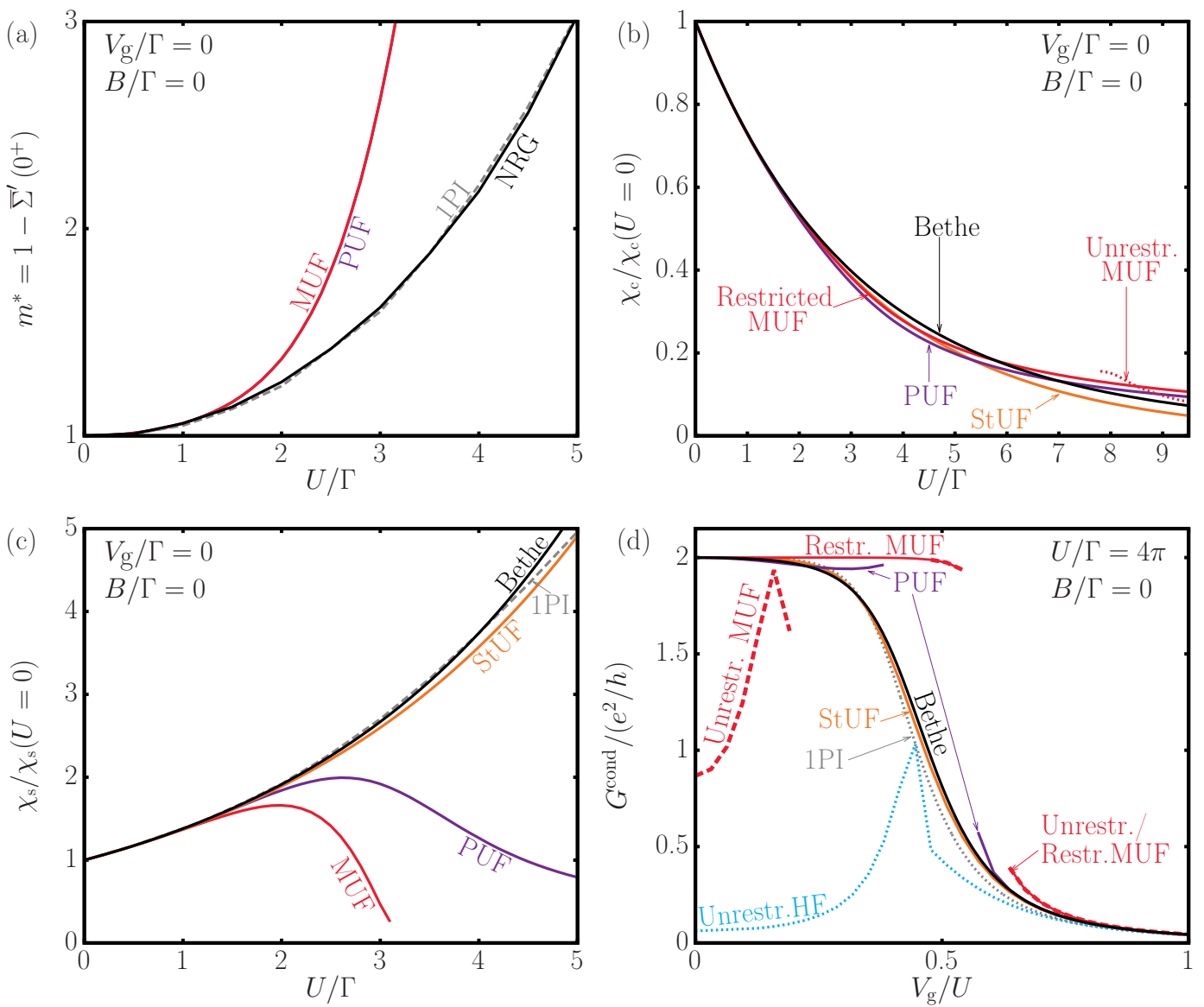

FIG. 4: (Color online) Numerical data for the PUF, StUF and MUF approximations in comparison to the same NRG, Bethe and 1PI fRG curves as in Fig. 2 (a) For the effective mass, the PUF and MUF curves are nearly indistinguishable in this plot. (b) For the charge susceptibility, the flow of unrestricted MUF does not come to an end for the intermediate regime $U / \Gamma \approx \pi \ldots 8$. (c) For the spin susceptibility, neither the flow of restricted MUF nor that of unrestricted MUF comes to an end beyond the critical interaction $U>\pi \Gamma$. (d) For the conductance, the flow does not come to an end (except for StUF) for gate voltages around the plateau edge at such a high interaction strength.

For the conductance, we observe a problem that is shared by the PUF and the restricted and unrestricted MUF schemes: The flow does not come to an end for values of $U / \Gamma$ around the edge of the conductance plateau. Note that the plateau is calculated at a large $U / \Gamma=4 \pi$. Tuning $U$ to smaller values lifts this problem. The points that are calculated show a tendency of all three methods to enlarge the plateau and to make the fall-off at the edge sharper than in the exact Bethe ansatz solution.

In summary, we find that the unphysical properties of the unrestricted and restricted Hartree-Fock starting points constitute a major problem for the MUF approximation. The unrestricted MUF scheme has proven to be not trustworthy for $U>U_{\text {crit }}$. The restricted MUF scheme performs better and makes it possible to pass $U_{\text {crit }}$ for $B=0$. The results are comparable to those of the PUF approximation.

\section{E. Results for StUF}

In this section, we discuss the numerical results for the StUF approximation. The corresponding data are as well shown in Fig. 4.

No reasonable effective mass can be calculated for this scheme, as the derivative of $\bar{\Sigma}_{\sigma}(\nu)$ with respect to $\nu$ is zero. The other observables, however, agree remarkably well with the exact results. This holds in particular for the conductance data which is even more remarkable at this large $U / \Gamma=4 \pi$. With 1PI fRG employing a static flow of the 1PI two-particle vertex [cf. Fig. 4(d) and Ref. 41, one is already able to obtain agreement with the exact curve at surprisingly large $U / \Gamma$ but StUF even outperforms this scheme.

The good performance of the StUF scheme is surprising for three reasons. First, it constitutes a lower order truncation to the 2PI fRG than the PUF or MUF scheme. Second, the computational effort needed for solving the scheme is marginal. Third, we find that it 
does not produce good results for the quantum anharmonic oscillator. $\underline{45}$

For $B=0$, we can gain analytical insight in the scaling behavior of the renormalized single-particle energy. Let us introduce the dimensionless renormalized level position

$$
f_{\lambda}=\frac{\epsilon_{\bar{\sigma}}+\bar{\Sigma}_{\bar{\sigma}, \lambda}^{\mathrm{StUF}}}{\Gamma} .
$$

We use $U_{\lambda}=\lambda U$ and set $u=\frac{U}{\pi \Gamma}$, to obtain [cf. Eq. 87] ]

$$
\dot{f}_{\lambda}=-u \operatorname{atan}\left(f_{\lambda}\right)
$$

with initial condition $f_{\lambda_{\mathrm{i}}}=\frac{V_{\mathrm{g}}}{\Gamma}=: v_{\mathrm{g}}$. If $v_{\mathrm{g}}=0$, the solution is $f_{\lambda}=0$. For $v_{\mathrm{g}} \neq 0$, separation of variables yields

$$
\int_{v_{g}}^{f_{\lambda}} \frac{d x}{\operatorname{atan}(x)}=-u \lambda
$$

The integral over $\frac{1}{\operatorname{atan}(x)}$ yields a scaling of $f_{\lambda_{\mathrm{f}}}(u) \sim$ $v_{\mathrm{g}} e^{-u} \sim e^{-\frac{1}{\pi} \frac{U}{\Gamma}}$. The (actual) Kondo temperature $T_{\mathrm{K}}$ scales $\sim e^{-\frac{\pi}{8} \frac{U}{\Gamma}}$. This means that the StUF approximation correctly predicts an exponential scaling with the interaction strength but yields the wrong prefactor $\frac{1}{\pi}$ instead of $\frac{\pi}{8}$. Lowest order 1PI vertex expansion Matsubara fRG (without flow of the two-particle vertex) also predicts $\sim e^{-\frac{1}{\pi} \frac{U}{\Gamma}}$ but reproduces the conductance plateau much worse than the StUF approximation [cf. Fig. 4(d) to Fig. 3 in Ref. 41 .

\section{F. Results for CUF and CF}

In this section, we discuss the results for the CUF and the $\mathrm{CF}$ approximation. Figure 5 shows the same observables as above for these two schemes.

Let us start by discussing the CF approximation. Like PUF and MUF, it reproduces the exact curves well only up to $U / \Gamma \approx 1 \ldots 2$. For the effective mass and the charge susceptibility, the curves are close to plain and selfconsistent second order perturbation theory results. $\frac{34138}{}$ This is not surprising. By construction the flow equation contains the required terms to generate plain second order perturbation theory but not enough to generate self-consistent second order perturbation theory. For the spin susceptibility, plain and self-consistent second order perturbation theory curves lie below the exact curve. The $\mathrm{CF}$ curve, in contrast, lies above. We conjecture that the self-consistent nature of the lowest order term in the CF scheme induces a strong influence of the self-consistent Hartree-Fock solution (which also lies above the exact curve). For the conductance, the $\mathrm{CF}$ approximation is able to produce reasonable data for all gate voltages.

Turning to the CUF method, we find that indeed the results of PUF can be improved for the effective mass, spin and charge susceptibility by fine-tuning the value of
$\Lambda$. With $\Lambda=2 \Gamma$ we are able to push the boundary for which the CUF data agree acceptably well with the exact results for all observables up to $U / \Gamma \approx 2 \ldots 3$. For the spin susceptibility, we see that further increasing $\Lambda$ to $4 \Gamma$ improves the agreement even more. For the effective mass however, the CUF curve for $\Lambda=4 \Gamma$ intersects the exact curve at $U / \Gamma \approx 3$ but deviates conceivably from the exact curve for larger and smaller (!) interaction strength. We conclude that we can optimize $\Lambda$ in an observable- and $U$-dependent manner such that we generate agreement to the exact curve. This is only partially satisfactory. We would have preferred the existence of an optimal choice of $\Lambda$ that yields agreement to the exact curves over a large range of $U$ for all observables.

\section{CONCLUSION}

In this paper we investigated how the $U$-flow fRG can be used to construct $\Phi$-derivable approximations, and how different $U$-flow approximations perform in computing typical observables of the Anderson impurity model in equilibrium.

Concerning the first question we found it helpful to address the flow of the Luttinger Ward functional $\Phi[G]$ and the flow of the physical values $\Phi^{(n)}[\bar{G}]$ of its vertex functions separately. We have seen that elementary truncations of the flow of the functional lead indeed to approximate $\Phi$ 's that are invariant under symmetry transformations and thus define $\Phi$-derivable (conserving) approximations. In the lowest order truncation we rederived the self-consistent Hartree-Fock approximation while the next higher truncation (cfRG) led to a $\Phi$ that closely resembles that of the FLEX approximation. In this sense, the fRG did not provide an approximation of a fundamentally new structure. In particular, it can again be understood as a diagrammatic approximation to $\Phi$, except for prefactors. This might change in higher order truncations, whose solution is however analytically quite involved and numerically inaccessible. It is remarkable that the analytic integration of the flow in the studied truncation yielded a result completely independent of the chosen flow parameter. This resembles the observation of Ref. 20 that the result of truncated $C$-flow for the physical vertex functions is independent of the flow parameter, namely given by self-consistent perturbation theory. As for the $C$-flow, we conclude that for models with infrared divergencies in perturbation theory the cfRG is only applicable if the resulting diagrammatic resummation or the self-consistency has a regularizing effect.

Next we studied the flow of the physical values $\Phi^{(n)}[\bar{G}]$ of the vertex functions. It is described by a coupled hierarchy of flow equations that is infinite even when the hierarchy for the functional $\Phi[G]$ has been truncated. Truncating in turn the hierarchy for the physical values will in general lead to a non- $\Phi$-derivable approximation. Therefore we could not obtain new $\Phi$-derivable fRG approximations from the flow of the physical values alone; 

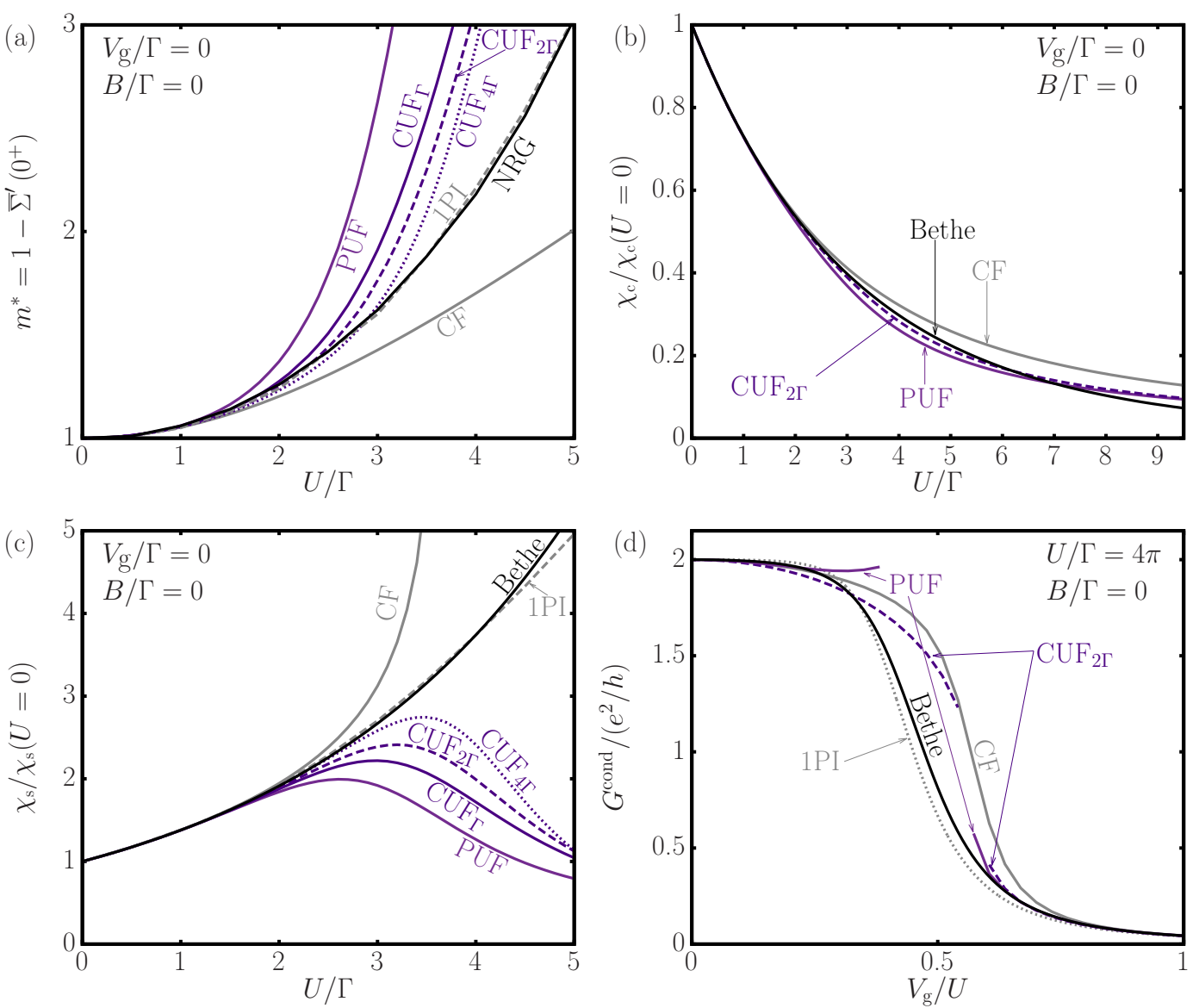

FIG. 5: (Color online) Numerical data for the CUF and the CF approximations in comparison to the same PUF, NRG, Bethe and 1PI fRG curves as in Fig. 22 For (a) the effective mass and (c) the spin susceptibility, we show CUF data for $\Lambda / \Gamma=1,2,4$. For (b) the charge susceptibility and (d) the conductance, we only show $\Lambda / \Gamma=2$ data.

the flow of the whole functional seems to be required. By truncating the new hierarchy we recovered indeed the plain and modified $U$-flow approximations of Ref. 20 (PUF and MUF) as non- $\Phi$-derivable approximations to the $\Phi$-derivable cfRG-approximation. We demonstrated explicitly that they are not thermodynamically consistent by comparing numerical results for the impurity occupancy obtained from different approaches. We truncated the hierarchy for the physical values also on the lowest order level and uncovered a simple static non- $\Phi$ derivable $U$-flow scheme (StUF) that was not noticed in Ref. 20.

We tested the different approximation schemes by computing typical observables of the equilibrium Anderson impurity model. Compared to 1PI fRG approximations the results are in general rather poor. For cfRG and PUF they reflect the kinship with FLEX, a method that is known to be of limited usefulness for the model at hand ${ }^{38}$ Based on an analytic prediction by Hamann 22 for a similar approximation we expected an $\exp \left(c U^{2}\right)$ behavior of the approximate effective mass. This is not confirmed by the data, not even for the very approximation analyzed by Hamann. We consider it improbable that errors in our numerics are the reason for the discrepancy. Our FLEX data coincide with published ones,$\frac{38}{38}$ and data for Hamann's approximation can be generated by changing only a few prefactors in the code. Further work is required to understand the discrepancy.

Concerning the modified $U$-flow, the artifacts introduced by the Hartree-Fock initial condition proved to constitute a major obstacle for the flow. Neither the spin symmetry breaking of the unrestricted nor the negative spin susceptibility of the restricted Hartree-Fock starting point were overcome by the RG flow. In contrast, they impeded the convergence of the numerical flow.

Comparing the numerical errors of the different $U$ flow schemes to those of self-consistent perturbation theory (which corresponds to straightforwardly truncated $C$ flow) we considered it conceivable that a combination of both methods might improve the approximation quality. Therefore we devised the $\mathrm{CUF}_{\Lambda}$ approximation, where the parameter $\Lambda$ allows for a smooth interpolation between the PUF approximation and an appropriate (non$\Phi$-derivable) $C$-flow truncation. We found that the range of validity of the approximation can indeed be extended for all discussed observables, however only to still mod- 
erate $U / \Gamma=2 \ldots 3$.

In contrast to the more elaborate schemes, the simple static variant StUF performed remarkably well, in particular in regard of the marginal computational effort it requires. It describes the linear conductance as function of the gate voltage better than any $1 \mathrm{PI}$ fRG method that has been applied to the problem. Similar to the static 1PI fRG without flow of the two-particle vertex, it allows to extract analytically a characteristic scale $\exp [-U /(\pi \Gamma)]$, where only the prefactor of the exponent differs from the exact Kondo temperature $\sim \exp [-\pi U /(8 \Gamma)]$. In view of this success it is an interesting question, whether the StUF approximation can be extended to higher order truncations in some other systematic way than studied here.

\section{Acknowledgements}

We thank D. Manske for helpful discussions on the FLEX approximation, S. Andergassen for her comments on a preprint version of the paper, and N. Dupuis for stimulating comments regarding the spin symmetry breaking in the MUF scheme. We acknowledge support by the Deutsche Forschungsgemeinschaft via the Research Training Group 1995 "Quantum many-body methods in condensed matter systems".

\section{Appendix A: Derivation of implementable equations}

In this appendix, we present more details on how to derive the self-consistency or flow equations in a form that exploits the symmetries and conservation laws specific for the Anderson impurity model. These equations can then serve as a starting point for the numerical implementation.

\section{Reducing the number of indices}

Like the interaction $U^{(2)}$ (cf. Sec. V A), all four-point functions $B=\Pi, \Upsilon, \ldots$ turn out to be sparse for the Anderson model. Moreover, they have components which are connected by symmetry. We want to refer to the components in a suitably reduced form. For this purpose, we group the four $y=(c, \sigma)$-indices together in pairs (the first two and the latter two). Such a pair may never take the index combination $\underset{\sigma \sigma}{c c}$ because this would correspond to a double creation/annihilation of a spin- $\sigma$ electron on the dot (this statement does not hold for $B^{\mathrm{R}}$ ). This leaves the following set of index combinations which are allowed for the pairs:

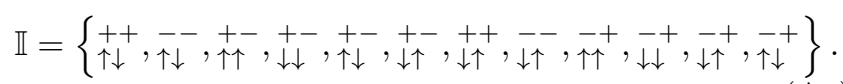

Let $s_{1}, s_{2}$ be such indices $\in \mathbb{I}$. Then, we can refer to all non-zero components of $B$ via $B_{s_{1} s_{2}, n_{1} n_{1}^{\prime} n_{2} n_{2}^{\prime}}$. As a side-note, the symmetry $B_{s_{1} s_{2}, n_{1} n_{1}^{\prime} n_{2} n_{2}^{\prime}}=B_{s_{2} s_{1}, n_{2} n_{2}^{\prime} n_{1} n_{1}^{\prime}}$ holds if $B=B^{\mathrm{T}}$. However, this is not always the case (e.g. $\Upsilon \neq \Upsilon^{\mathrm{T}}$ ). Thus, we do not use this property in the following considerations. We define an operation ${ }^{\sim}$ on $s$ via

$$
\widetilde{s}=\widetilde{\left(\begin{array}{l}
c_{1} c_{2} \\
\sigma_{1} \sigma_{2}
\end{array}\right)}=\left(\begin{array}{l}
c_{2} c_{1} \\
\sigma_{2} \sigma_{1}
\end{array}\right) .
$$

Then, the relation $B_{s_{1} s_{2}, n_{1} n_{1}^{\prime} n_{2} n_{2}^{\prime}}=-B_{\widetilde{s}_{1} s_{2}, n_{1}^{\prime} n_{1} n_{2} n_{2}^{\prime}}$ holds in general [cf. Eq. (8)]. The first six indices and the last six indices in the set $\mathbb{I}$ are connected via the

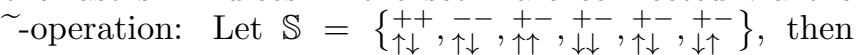
$\mathbb{I}=\mathbb{S} \cup \widetilde{\mathbb{S}}$. If we base a $12 \times 12$-matrix notation of $B$ on the order of indices as chosen above, each matrix $B$ can be written in terms of a $6 \times 6$-matrix $\underline{B}$ :

$$
B_{s_{1} s_{2}, n_{1} n_{1}^{\prime} n_{2} n_{2}^{\prime}}=\left(\begin{array}{cc}
\underline{B}_{n_{1} n_{1}^{\prime} n_{2} n_{2}^{\prime}} & -\underline{B}_{n_{1} n_{1}^{\prime} n_{2}^{\prime} n_{2}} \\
-\underline{B}_{n_{1}^{\prime} n_{1} n_{2} n_{2}^{\prime}} & \underline{B}_{n_{1}^{\prime} n_{1} n_{2}^{\prime} n_{2}}
\end{array}\right)_{s_{1} s_{2}}
$$

Obviously, it is sufficient to work with the underlined matrices. For example, a contraction of $s$-indices $\in \mathbb{I}$ is the same as a contraction of $S$-indices $\in \mathbb{S}$ taking into account a factor of $\frac{1}{2}$, i.e. $\frac{1}{2} \sum_{s} \leftrightarrow \sum_{S}$. As an example for an underlined matrix, we provide the interaction in this notation:

$$
\begin{array}{r}
\underline{U}_{S_{1} S_{2}, n_{1} n_{1}^{\prime} n_{2} n_{2}^{\prime}}=\beta \delta_{n_{1}+n_{1}^{\prime}+n_{2}+n_{2}^{\prime}, 0} \\
\times\left(\begin{array}{cccccc}
0 & -U & 0 & 0 & 0 & 0 \\
-U & 0 & 0 & 0 & 0 & 0 \\
0 & 0 & 0 & U & 0 & 0 \\
0 & 0 & U & 0 & 0 & 0 \\
0 & 0 & 0 & 0 & 0 & -U \\
0 & 0 & 0 & 0 & -U & 0
\end{array}\right)_{S_{1} S_{2}} .
\end{array}
$$

This matrix is block-diagonal consisting of three $2 \times 2$ matrices. It can be shown that this is a general feature for all underlined matrices $\underline{B}$. This is a consequence of particle-number conservation and spin conservation which is fulfilled by propagation and interaction in the Anderson model. Particle-number conservation implies that the number of $c$ 's equal to + must be even and so must the number of $c=-$. It does, however, not imply that the sum of all $c$ 's must be 0 . This holds only for vertex-like (e.g. $U$ ) or propagator-like (e.g. $\Pi$ ) quantities. Combinations of these quantities which are $I$-like (e.g. $\Upsilon$ ) may have all $c$ 's equal to + or equal to - [see e.g. Eq. A15]. Spin-conservation implies that $c_{1} \sigma_{1}+c_{1}^{\prime} \sigma_{1}^{\prime}$ must be equal to $\pm\left(c_{2} \sigma_{2}+c_{2}^{\prime} \sigma_{2}^{\prime}\right)$. The sign depends on whether the quantity is vertex-, propagatoror $I$-like. For illustration let us consider two examples. A $\left(S_{1} S_{2}=\underset{\uparrow \downarrow}{++\uparrow}+\underset{\uparrow}{++-}\right)$-component would violate particlenumber conservation. A $\left(S_{1} S_{2}=\underset{\uparrow \uparrow \uparrow \downarrow}{+-+-}\right)$-component would violate spin conservation.

\section{Calculating $\bar{\Upsilon}$ for the Anderson model}

In this section, we calculate $\bar{\Upsilon}$ for the Anderson model. We will need this quantity in (almost) all methods. 
We use Eq. (9) to find

$$
\begin{aligned}
\bar{\Pi}_{n_{1} n_{1}^{\prime} n_{2} n_{2}^{\prime}}^{y_{1} y_{1}^{\prime} y_{2} y_{2}^{\prime}}= & \beta^{2} \delta_{n_{1}^{\prime}+n_{2}, 0} \delta_{n_{1}+n_{2}^{\prime}, 0} \bar{G}_{n_{1}^{\prime}}^{y_{1}^{\prime} y_{2}} \bar{G}_{n_{1}}^{y_{1} y_{2}^{\prime}} \\
& -\beta^{2} \delta_{n_{1}+n_{2}, 0} \delta_{n_{1}^{\prime}+n_{2}, 0} \bar{G}_{n_{1}}^{y_{1} y_{2}} \bar{G}_{n_{1}^{\prime}}^{y_{1}^{\prime} y_{2}^{\prime}} .
\end{aligned}
$$

With

$$
U_{n_{1} n_{1}^{\prime} n_{2} n_{2}^{\prime}}^{y_{1} y_{2}^{\prime} y_{2}^{\prime}}=\beta \delta_{n_{1}+n_{1}^{\prime}+n_{2}+n_{2}^{\prime}, 0} U^{y_{1} y_{1}^{\prime} y_{2} y_{2}^{\prime}},
$$

we then find

$$
(U \cdot \bar{\Pi})_{n_{1} n_{1}^{\prime} n_{2} n_{2}^{\prime}}^{y_{1} y_{1}^{\prime} y_{2} y_{2}^{\prime}}=\beta \delta_{n_{1}+n_{1}^{\prime}-n_{2}-n_{2}^{\prime}, 0} \bar{\Psi}_{-n_{2},-n_{2}^{\prime}}^{y_{1} y_{1}^{\prime} y_{2} y_{2}^{\prime}}
$$

in which

$$
\bar{\Psi}_{-n_{2},-n_{2}^{\prime}}^{y_{1} y_{1}^{\prime} y_{2} y_{2}^{\prime}}=-\sum_{y_{3} y_{3}^{\prime}} U^{y_{1} y_{1}^{\prime} y_{3} y_{3}^{\prime}} \bar{G}_{-n_{2}}^{y_{3} y_{2}} \bar{G}_{-n_{2}^{\prime}}^{y_{3}^{\prime} y_{2}^{\prime}} .
$$

We then prove

$$
\begin{aligned}
& {[(-U \cdot \bar{\Pi}) \cdot(-U \cdot \bar{\Pi})]_{n_{1} n_{1}^{\prime} n_{2} n_{2}^{\prime}}^{y_{1} y_{1}^{\prime} y_{2} y_{2}^{\prime}}} \\
& =\beta \delta_{n_{1}+n_{1}^{\prime}-n_{2}-n_{2}^{\prime}, 0} \frac{1}{2 \beta} \sum_{y_{3} y_{3}^{\prime} n_{3}} \bar{\Psi}_{-n_{3}, n_{3}-n_{2}-n_{2}^{\prime}}^{y_{1} y_{1}^{\prime} y_{3} y_{3}^{\prime}} \bar{\Psi}_{-n_{2},-n_{2}^{\prime}}^{y_{3} y_{3}^{\prime} y_{2} y_{2}^{\prime}}
\end{aligned}
$$

Let us now introduce some notations concerning the space of $y$-indices only: $\mathbb{1}^{y_{1} y_{1}^{\prime} y_{2} y_{2}^{\prime}}=\delta_{y_{1} y_{2}} \delta_{y_{1}^{\prime} y_{2}^{\prime}}-\delta_{y_{1} y_{2}^{\prime}} \delta_{y_{1}^{\prime} y_{2}}$ and $(A \circ B)^{y_{1} y_{1}^{\prime} y_{2} y_{2}^{\prime}}=\frac{1}{2} \sum_{y_{3} y_{3}^{\prime}} A^{y_{1} y_{1}^{\prime} y_{3} y_{3}^{\prime}} B^{y_{3} y_{3}^{\prime} y_{2} y_{2}^{\prime}}$. Then, we show by induction that

$$
\begin{aligned}
& {\left[(-U \cdot \bar{\Pi})^{k} \cdot(-U \cdot \bar{\Pi})\right]_{n_{1} n_{1}^{\prime} n_{2} n_{2}^{\prime}}^{y_{1} y_{1}^{\prime} y_{2} y_{2}^{\prime}} } \\
= & -\beta \delta_{n_{1}+n_{1}^{\prime}-n_{2}-n_{2}^{\prime}, 0} \\
& \times\left\{\left[-\frac{1}{\beta} \sum_{n} \bar{\Psi}_{-n, n-n_{2}-n_{2}^{\prime}}\right]^{\circ k} \circ \bar{\Psi}_{-n_{2},-n_{2}^{\prime}}\right\}
\end{aligned}
$$

On the right-hand side, we introduced ${ }^{\circ} k$ to refer to the $k$-fold o operation. We define the abbreviation

$$
\widetilde{\Psi}_{m=n_{2}+n_{2}^{\prime}}=\frac{1}{\beta} \sum_{n} \bar{\Psi}_{-n, n-n_{2}-n_{2}^{\prime}} .
$$

Making use of the geometric series, we now find

$$
\begin{aligned}
\bar{\Upsilon}_{n_{1} n_{1}^{\prime} n_{2} n_{2}^{\prime}}^{y_{1} y_{1}^{\prime} y_{2} y^{\prime}}= & \beta \delta_{n_{1}+n_{1}^{\prime}-n_{2}-n_{2}^{\prime}, 0} \\
& \times\left\{\left[\mathbb{1}+\widetilde{\Psi}_{n_{2}+n_{2}^{\prime}}\right]^{\circ(-1)} \circ \bar{\Psi}_{-n_{2},-n_{2}^{\prime}}\right\}^{y_{1} y_{1}^{\prime} y_{2} y_{2}^{\prime}} .
\end{aligned}
$$

Here, the inverse ${ }^{\circ(-1)}$ is to be understood with respect to the $\circ$ operation in the space of $y$-indices. For this inversion, we resort to the matrix notation introduced in Sec. A 1 .

Before performing this inversion, we reduce the frequency structure: In Eq. A12, the fourth index $n_{2}^{\prime}$ is determined by the $\delta$-function. Thus, $\bar{\Upsilon}_{n_{1} n_{1}^{\prime} n_{2} n_{2}^{\prime}}^{y_{1} y_{1}^{\prime} y_{2} y^{\prime}}$ actually depends on three frequency indices only. Conveniently, $\bar{\Upsilon}$ turns out to be needed only in a form in which the third index is always summed over independently. Thus, this summed $\bar{\Upsilon}$ depends only on the first and second indices. In fact, it turns out to only depend on the sum of the two indices and we define:

$$
\begin{aligned}
\widetilde{\Upsilon}_{n_{1}+n_{1}^{\prime}}^{y_{1} y_{1}^{\prime} y_{2} y_{2}^{\prime}} & =\frac{1}{\beta} \sum_{n_{2}} \bar{\Upsilon}_{n_{1}, n_{1}^{\prime}, n_{2}}^{y_{1} y_{1}^{\prime} y_{2} y_{2}^{\prime}} \\
& =\left\{\left[\mathbb{1}+\widetilde{\Psi}_{n_{1}+n_{1}^{\prime}}\right]^{\circ(-1)} \circ \widetilde{\Psi}_{n_{1}+n_{1}^{\prime}}\right\}^{y_{1} y_{1}^{\prime} y_{2} y_{2}^{\prime}} .
\end{aligned}
$$

Now, let us turn to the inversion of $\left[\mathbb{1}+\widetilde{\Psi}_{n_{1}+n_{1}^{\prime}}\right]$. Using Eqs. A8 and (A11), we determine $\widetilde{\Psi}_{m}^{S_{1} S_{2}}$ for indices $S_{1}, S_{2} \in \mathbb{S}=\left\{\begin{array}{c}++ \\ \uparrow \downarrow\end{array}, \underset{\uparrow \downarrow}{-}, \underset{\uparrow \uparrow}{+-},+\underset{\downarrow \downarrow}{+-}, \underset{\uparrow \downarrow}{+-}, \underset{\downarrow \uparrow}{+-}\right\}:$

$$
\widetilde{\Psi}_{m}^{S_{1} S_{2}}=\left(\begin{array}{cccccc}
\widetilde{\Psi}_{m}^{\mathrm{p}} & 0 & 0 & 0 & 0 & 0 \\
0 & \widetilde{\Psi}_{m}^{\mathrm{p} *} & 0 & 0 & 0 & 0 \\
0 & 0 & 0 & \widetilde{\Psi}_{m}^{\mathrm{d} \uparrow} & 0 & 0 \\
0 & 0 & \widetilde{\Psi}_{m}^{\mathrm{d} \downarrow} & 0 & 0 & 0 \\
0 & 0 & 0 & 0 & \widetilde{\Psi}_{m}^{\mathrm{x} \uparrow} & 0 \\
0 & 0 & 0 & 0 & 0 & \widetilde{\Psi}_{m}^{\mathrm{x} \downarrow}
\end{array}\right)_{S_{1} S_{2}}
$$

Here, we used the abbreviations

$$
\begin{aligned}
& \widetilde{\Psi}_{m}^{\mathrm{p}}=\widetilde{\Psi}_{\uparrow \downarrow \uparrow \downarrow, m}^{++++}=U \frac{1}{\beta} \sum_{n} \bar{G}_{\uparrow,-n}^{-} \bar{G}_{\downarrow, n-m}^{-}, \\
& \widetilde{\Psi}_{m}^{\mathrm{d} \uparrow}=\widetilde{\Psi}_{\uparrow \uparrow \downarrow \downarrow, m}^{+-+-}=-U \frac{1}{\beta} \sum_{n} \bar{G}_{\downarrow, n}^{-} \bar{G}_{\downarrow, n+m}^{-} \in \mathbb{R}, \\
& \widetilde{\Psi}_{m}^{\mathrm{d} \downarrow}=\widetilde{\Psi}_{\downarrow \downarrow \uparrow \uparrow, m}^{+-+-}=-U \frac{1}{\beta} \sum_{n} \bar{G}_{\uparrow, n}^{-} \bar{G}_{\uparrow, n+m}^{-} \in \mathbb{R}, \\
& \widetilde{\Psi}_{m}^{\mathrm{x} \uparrow}=\widetilde{\Psi}_{\uparrow \downarrow \uparrow \downarrow, m}^{+-+-}=U \frac{1}{\beta} \sum_{n} \bar{G}_{\uparrow, n}^{-} \bar{G}_{\downarrow, n+m}^{-}, \\
& \widetilde{\Psi}_{m}^{\mathrm{x} \downarrow}=\widetilde{\Psi}_{\downarrow \uparrow \downarrow \uparrow, m}^{+-+-}=\widetilde{\Psi}_{m}^{\mathrm{x} \uparrow *} .
\end{aligned}
$$

The labeling of these abbreviations is inspired by the role of the corresponding components in, for example, the FLEX ladder summations. There are particle-particle and direct particle-hole as well as exchange particle-hole contributions. The extra-labeling with $\uparrow$ or $\downarrow$ refers to which $\sigma$-component of the self-energy is affected by the contribution.

Because of its block-diagonal structure, the inverse of $\left[\mathbb{1}+\widetilde{\Psi}_{m}\right]$ is easily computed. Multiplying the result with $\widetilde{\Psi}_{m}$ according to Eq. A13 yields

$$
\widetilde{\Upsilon}_{m}^{S_{1} S_{2}}=\left(\begin{array}{cccccc}
\tilde{\Upsilon}_{m}^{\mathrm{p}} & 0 & 0 & 0 & 0 & 0 \\
0 & \widetilde{\Upsilon}_{m}^{\mathrm{p} *} & 0 & 0 & 0 & 0 \\
0 & 0 & \widetilde{\Upsilon}_{m}^{\bar{d}} & \widetilde{\Upsilon}_{m}^{\mathrm{d} \uparrow} & 0 & 0 \\
0 & 0 & \widetilde{\Upsilon}_{m}^{\mathrm{d} \downarrow} & \widetilde{\Upsilon}_{m}^{\bar{d}} & 0 & 0 \\
0 & 0 & 0 & 0 & \widetilde{\Upsilon}_{m}^{\mathrm{x} \uparrow} & 0 \\
0 & 0 & 0 & 0 & 0 & \widetilde{\Upsilon}_{m}^{\mathrm{x} \downarrow}
\end{array}\right)_{S_{1} S_{2}}
$$


Here, we used the following abbreviations in which again the labels are inspired by the role of the component in the calculation of the self-energy:

$$
\begin{aligned}
& \widetilde{\Upsilon}_{m}^{\mathrm{p}}=\widetilde{\Upsilon}_{\uparrow \downarrow \uparrow \downarrow, m}^{++++}=\widetilde{\Psi}_{m}^{\mathrm{p}}\left[1+\widetilde{\Psi}_{m}^{\mathrm{p}}\right]^{-1} \\
& \widetilde{\Upsilon}_{m}^{\mathrm{d}}=\widetilde{\Upsilon}_{\uparrow \uparrow \uparrow \uparrow, m}^{+-+-}=-\widetilde{\Psi}_{m}^{\mathrm{d} \uparrow} \widetilde{\Psi}_{m}^{\mathrm{d} \downarrow}\left[1-\widetilde{\Psi}_{m}^{\mathrm{d} \uparrow} \widetilde{\Psi}_{m}^{\mathrm{d} \downarrow}\right]^{-1} \in \mathbb{R} \\
& \widetilde{\Upsilon}_{m}^{\mathrm{d} \uparrow}=\widetilde{\Upsilon}_{\uparrow \uparrow \downarrow \downarrow, m}^{++-+-}=\widetilde{\Psi}_{m}^{\mathrm{d} \uparrow}\left[1-\widetilde{\Psi}_{m}^{\mathrm{d} \uparrow} \widetilde{\Psi}_{m}^{\mathrm{d} \downarrow}\right]^{-1} \in \mathbb{R}, \\
& \widetilde{\Upsilon}_{m}^{\mathrm{d} \downarrow}=\widetilde{\Upsilon}_{\downarrow \downarrow \uparrow \uparrow, m}^{+++-}=\widetilde{\Psi}_{m}^{\mathrm{d} \downarrow}\left[1-\widetilde{\Psi}_{m}^{\mathrm{d} \uparrow} \widetilde{\Psi}_{m}^{\mathrm{d} \downarrow}\right]^{-1} \in \mathbb{R} \\
& \widetilde{\Upsilon}_{m}^{\mathrm{x} \uparrow}=\widetilde{\Upsilon}_{\uparrow \downarrow \uparrow \downarrow, m}^{+++-}=\widetilde{\Psi}_{m}^{\mathrm{x} \uparrow}\left[1+\widetilde{\Psi}_{m}^{\mathrm{x} \uparrow}\right]^{-1} \\
& \widetilde{\Upsilon}_{m}^{\mathrm{x} \downarrow}=\widetilde{\Upsilon}_{\downarrow \uparrow \downarrow \uparrow, m}^{+++-}=\widetilde{\Upsilon}_{m}^{\mathrm{x} \uparrow *}
\end{aligned}
$$

Note that the complex conjugation relation $\bar{\Sigma}_{\sigma, n}^{c *}=$ $\bar{\Sigma}_{\sigma,-n}^{c}$ holds. For its proof, consider the following reasoning: In the derivation of the flow (or self-consistency) equations it was assumed to hold. These equations are found to not lead to a violation of the relation. Furthermore, we start with initial conditions (or guesses) which do not violate the relation. Consequently, the relation is self-consistently fulfilled. The relation also implies $\widetilde{\Psi}_{m}^{i}=\widetilde{\Psi}_{-m}^{i *}$ and $\widetilde{\Upsilon}_{m}^{i}=\widetilde{\Upsilon}_{-m}^{i *}$.

\section{Self-consistency equations for the conserving schemes}

The goal of this section is to provide self-consistency equations for the FLEX and the cfRG approximation that can be used for the numerical implementation. For FLEX, we insert $\alpha=\left(c, \sigma, \nu_{n}\right)$ in Eq. 79 and exploit the symmetries and the sparseness of components to find

$$
\begin{aligned}
\bar{\Sigma}_{\sigma, n}^{\mathrm{FLEX}+}=\frac{U}{\beta} \sum_{m}\{[ & \left.\frac{2}{3} \widetilde{\Psi}_{m}^{\mathrm{p}}-\widetilde{\Upsilon}_{m}^{\mathrm{p}}\right] \bar{G}_{\bar{\sigma}, n-m}^{-} \\
+ & {\left[\widetilde{\Upsilon}_{m}^{\mathrm{d} \sigma}-\frac{2}{3} \widetilde{\Psi}_{m}^{\mathrm{d} \sigma}\right] \bar{G}_{\sigma, m-n}^{-} } \\
& \left.+\left[\frac{2}{3} \widetilde{\Psi}_{m}^{\mathrm{x} \sigma}-\widetilde{\Upsilon}_{m}^{\mathrm{x} \sigma}\right] \bar{G}_{\bar{\sigma}, m-n}^{-}\right\} \\
+ & \frac{U}{\beta} \sum_{n^{\prime}} \bar{G}_{\bar{\sigma}, n^{\prime}}^{-} e^{-i \nu_{n^{\prime}} 0^{+}}-\frac{U}{2}
\end{aligned}
$$

The convergence factor in the last line is necessary and a consequence of correct imaginary time ordering. It can be "canceled" with the addend $-\frac{U}{2}$. Remember that the second order diagram contribution

$$
-\left(\frac{U}{\beta}\right)^{2} \sum_{m, l} \bar{G}_{\sigma,-l}^{-} \bar{G}_{\bar{\sigma}, l-m}^{-} \bar{G}_{\bar{\sigma}, n-m}^{-}
$$

is included correctly in FLEX. In Eq. A27, it can be seen that the three channels contribute each $\frac{1}{3}$ of this contribution. This arises naturally from the charge index notation. We now perform the $T=0$ limit $\left(\frac{1}{\beta} \sum_{n} \rightarrow\right.$ $\left.\int_{-\infty}^{\infty} \frac{d \nu}{2 \pi}\right)$. Furthermore, we drop the charge index + on the self-energy and the - on the propagator and find Eq. (82). In this equation, we introduced constants $\kappa_{i}$ which must be chosen as $\kappa_{0}=\frac{2}{3}$ and $\kappa_{\mathrm{p}}=\kappa_{\mathrm{d}}=\kappa_{\mathrm{x}}=1$ for FLEX. The cfRG leads to the same form as shown in Eq. 82. We must then choose $\kappa_{0}=0$ and $\kappa_{\mathrm{p}}=\kappa_{\mathrm{d}}=$ $\kappa_{\mathrm{X}}=\frac{1}{3}$.

\section{Flow equations for the non-conserving schemes}

In this section, we specify the relevant equations for the implementation of the non-conserving schemes. We start out by discussing the PUF, StUF and MUF approximations and proceed then with the CUF and $\mathrm{CF}$ approximations.

For the PUF approximation, we find Eq. (83). The last term does not need a convergence factor because $\dot{\bar{G}}_{\sigma}^{\lambda}(\nu)$ goes as $\sim 1 / \nu^{2}$. The initial condition is $\bar{\Sigma}_{\sigma, \lambda_{\mathrm{i}}}^{\mathrm{PUF}}(\nu)=0$ (and $U_{\lambda_{\mathrm{i}}}=0$ ).

The flow equation for the StUF approximation reads as

$$
\dot{\bar{\Sigma}}_{\sigma, \lambda}^{\mathrm{StUF}}=\dot{U}_{\lambda} \int_{-\infty}^{\infty} \frac{d \nu^{\prime}}{\pi} \operatorname{Re}\left[\bar{G}_{\bar{\sigma}}^{\lambda}\left(\nu^{\prime}\right)\right]
$$

with initial condition $\bar{\Sigma}_{\sigma, \lambda_{i}}^{\mathrm{StUF}}=0$. As no frequency dependence is acquired in this scheme, we directly perform the frequency integral analytically and find Eq. (87).

The equation for the MUF approximation is Eq. (84. The non-zero initial condition is $\bar{\Sigma}_{\sigma, \lambda_{\mathrm{i}}}^{\mathrm{MUF}}(\nu)=\bar{\Sigma}_{\sigma}^{\mathrm{HF}}=$ $-\frac{U}{\pi} \operatorname{atan}\left[\left(\epsilon_{\bar{\sigma}}+\bar{\Sigma}_{\bar{\sigma}}^{\mathrm{HF}}\right) / \Gamma\right]$. For the PUF, StUF and MUF schemes, we take $U_{\lambda}=\lambda U, \lambda=0 \ldots 1$.

Now, we specify the flow equations for the CUF scheme. In this case all propagators depend explicitly on $\lambda\left[\right.$ in addition to their implicit dependence due to $\left.\bar{\Sigma}_{\sigma}^{\lambda}(\nu)\right]$ :

$$
\begin{aligned}
\bar{G}_{\sigma}^{-}(\nu) & =-\frac{1}{i \nu+\epsilon_{\sigma}+i \operatorname{sgn}(\nu) \Gamma+\bar{\Sigma}_{\sigma}^{\lambda}(\nu)^{*}} \\
& \rightarrow-\frac{\Theta(|\nu|-\lambda)}{i \nu+\epsilon_{\sigma}+i \operatorname{sgn}(\nu) \Gamma+\bar{\Sigma}_{\sigma}^{\lambda}(\nu)^{*}} .
\end{aligned}
$$

Because of the sharp frequency cut-off we can replace $\bar{\Pi} \cdot \dot{C}^{-1}$ by $\left.\bar{G}\right|_{\Theta \rightarrow \delta}$ when calculating the extra addend from Eq. (52). This $\delta$-function cancels the frequency integral and we find

$$
\begin{array}{r}
-\frac{U_{\lambda}}{2 \pi} \sum_{x= \pm \lambda}\left\{\left[\widetilde{\Psi}_{\lambda}^{\mathrm{x} \sigma}(\nu+x)-\widetilde{\Psi}_{\lambda}^{\mathrm{p}}(\nu-x)\right] \bar{G}_{\bar{\sigma}}^{\lambda}(x)\right. \\
\left.+\widetilde{\Psi}_{\lambda}^{\mathrm{d} \sigma}(\nu+x) \bar{G}_{\sigma}^{\lambda}(x)\right\}=: \Delta_{\sigma}^{\lambda}(\nu) .
\end{array}
$$

In total, we obtain the flow equation 85). Note that $\dot{\bar{\Sigma}}_{\sigma}^{\mathrm{PUF}}(\nu)$ represents symbolically what is specified in Eq. (83). However, we must now use the explicitly $\lambda$ dependent $\bar{G}_{\sigma}^{\lambda}(\nu)$ from Eq. (A30) and $U_{\lambda}$ from Eq. (53) 
in order to calculate the right-hand side. The initial condition for the CUF scheme is $\bar{\Sigma}_{\sigma, \lambda_{\mathrm{i}}}^{\mathrm{CUF}_{\Lambda}}(\nu)=0$.

The flow equation for the $\mathrm{CF}$ approximation is Eq. 86. In the second addend, we must apply the replacement $U_{\lambda} \rightarrow U$ on every level - also in the calculation of the components of $\widetilde{\Psi}_{\lambda}$. The numerical initial condition is $\bar{\Sigma}_{\lambda_{\mathrm{i}}^{\text {num }}, \sigma}^{\mathrm{CF}}(\nu)=0$.

\section{Appendix B: Details on our numerical implementation}

We work with two meshes for the frequencies: a grid of fermionic frequencies $\nu_{n}$ for the self-energy and a grid of bosonic frequencies $\omega_{m}$ for the auxiliary quantities $\widetilde{\Psi}$ and $\widetilde{\Upsilon}$. They are given by the geometric formulas $(n, m \geq$ $0)$

$$
\begin{aligned}
\nu_{n} & =d \nu \frac{\left(1+f_{\nu}\right)^{n}-1}{f_{\nu}} \\
\omega_{m} & =d \nu \frac{\left(1+f_{\omega}\right)^{m}-1}{f_{\omega}} .
\end{aligned}
$$

Note that $n, m$ now refer to grid points and not to Matsubara frequencies. Setting $\Gamma=1$, the following parameters must be externally specified: $d \nu, \nu_{\max }$ and $n_{\text {len }}$. Then, we set $m_{\text {len }}=2 n_{\text {len }}$ and $\omega_{\max }=\nu_{\max }^{2}$. Thus, the bosonic grid has twice as many points as the fermionic grid but also extends to much larger frequencies. We compute $f_{\nu}, f_{\omega}$ such that $d \nu\left[\left(1+f_{\nu}\right)^{n_{\text {len }}}-1\right] / f_{\nu}=\nu_{\max }$ and $d \nu\left[\left(1+f_{\omega}\right)^{m_{\text {len }}}-1\right] / f_{\omega}=\omega_{\max }$. The value of a quantity is determined by cubic interpolation if the frequency is not exactly on one of the grid points. $\bar{G}_{\sigma}(\nu)$ is an exception, see following.

We restrict the grids to non-negative frequencies. This is sufficient, since we can apply complex conjugation relations to express quantities at negative frequencies through their values at positive frequencies (cf. Appendix A 2. The advantages of this procedure are a reduced grid size and an accurate treatment of discontinuities at zero frequency. A discontinuity of $\widetilde{\Upsilon}$ at zero frequency results from the appearance of $\operatorname{sgn} \nu$ in the free propagator, cf. Eq. (58). A numerical interpolation close to discontinuities is difficult and avoided by our approach with a grid of non-negative frequencies only.

For both conserving and non-conserving schemes, we store $\bar{\Sigma}$ by separating the asymptotic value from the rest,

$$
\bar{\Sigma}_{\sigma}(\nu)=\left\{\begin{array}{ll}
\bar{\Sigma}_{\sigma}^{\mathrm{C}}+\bar{\Sigma}_{\sigma}^{\mathrm{D}}(\nu) & , 0 \leq \nu<\nu_{\max } \\
\bar{\Sigma}_{\sigma}^{\mathrm{C}} & , \nu>\nu_{\max }
\end{array},\right.
$$

in which $\bar{\Sigma}_{\sigma}^{\mathrm{C}}=\lim _{\nu \rightarrow \infty} \bar{\Sigma}_{\sigma}(\nu)$. Now, $\bar{G}_{\sigma}(\nu)$ can be computed for all $\nu$. If needed, $\bar{\Sigma}_{\sigma}^{\mathrm{D}}(\nu)$ is computed by cubic interpolation. Thus, integrals over integrands consisting purely of $\bar{G}_{\sigma}(\nu)$ can be calculated from $-\infty$ to $\infty$ (and not only on a finite range). The outer parts can be calculated analytically because the self-energy is taken as constant there. We make use of this for the calculation of $\widetilde{\Psi}$.

Splitting $\bar{\Sigma}_{\sigma}(\nu)$ up as in Eq. (B3) constitutes an approximation for finite $\nu_{\max }$. This allows to compute $\bar{G}_{\sigma}(\nu)$ for arbitrary $\nu$ with an error of $\mathcal{O}\left(\frac{1}{\nu_{\max }^{2}}\right)$. Performing $\omega$-integrations which formally go from $-\infty$ to $\infty$ only from $-\omega_{\max }$ to $\omega_{\max }$ also induces an error. By requiring $\omega_{\max } \sim \nu_{\max }^{2}$ we ensure that this error is as well of $\mathcal{O}\left(\frac{1}{\nu_{\max }^{2}}\right)$.

All flow equations in Appendix A 4 [except Eq. A29 for StUF] pose a self-consistency problem in each step of the flow because the right-hand side contains $\dot{\bar{G}}_{\sigma}^{\lambda}(\nu)$. This problem is easily solved because $\dot{\bar{G}}_{\sigma}^{\lambda}(\nu)$ always occurs only in a separate addend contributing to the frequency independent asymptotic value of the self-energy. By inserting the separation (B3) into the flow equation for $\bar{\Sigma}_{\sigma}^{\lambda}(\nu)$, we obtain separate equations for $\dot{\bar{\Sigma}}_{\sigma}^{\lambda, \mathrm{C}}$ and $\dot{\bar{\Sigma}}_{\sigma}^{\lambda, \mathrm{D}}(\nu)$. The right-hand side of the flow equation for $\dot{\bar{\Sigma}}_{\sigma}^{\lambda, \mathrm{D}}(\nu)$ then does not contain $\dot{\bar{G}}_{\sigma}^{\lambda}(\nu)$. Furthermore, the equation for $\dot{\bar{\Sigma}}_{\sigma}^{\lambda, \mathrm{C}}$ has the form $\dot{\bar{\Sigma}}_{\sigma}^{\lambda, \mathrm{C}}=A_{1}^{\sigma}+A_{2}^{\sigma} \dot{\bar{\Sigma}}_{\bar{\sigma}}^{\lambda, \mathrm{C}}$ in which $A_{1}^{\sigma}$ depends on $\dot{\Sigma}_{\bar{\sigma}}^{\lambda, \mathrm{D}}(\nu)$. The explicit solution of this equation is

$$
\dot{\bar{\Sigma}}_{\sigma}^{\lambda, \mathrm{C}}=\frac{A_{1}^{\sigma}+A_{2}^{\sigma} A_{1}^{\bar{\sigma}}}{1-A_{2}^{\sigma} A_{2}^{\bar{\sigma}}} .
$$

In each step of the flow, we thus proceed in the following manner: First, we compute $\dot{\bar{\Sigma}}_{\sigma}^{\lambda, \mathrm{D}}(\nu)$. Second, we calculate $A_{1}^{\sigma}$ and $A_{2}^{\sigma}$. Third, we calculate $\dot{\bar{\Sigma}}_{\sigma}^{\lambda, \mathrm{C}}$ by Eq. (B4).

Equation (B4) is suitable to analyze the divergence that occurs in the unrestricted MUF for $U$ moderately greater than $\pi \Gamma$, compare Sec.VID. For such $U$, the real part of the self-energy is almost constant $\operatorname{Re} \bar{\Sigma}_{\sigma}^{\lambda}(\nu) \approx \bar{\Sigma}_{\sigma}^{\lambda}$. In the unrestricted Hartree-Fock solution (for $V_{\mathrm{g}}=0=$ $B)$, the up and down self-energy take non-zero values of opposite sign, i.e. $\bar{\Sigma}_{\sigma}^{\lambda_{\mathrm{i}}}=\sigma \widetilde{h}$. This $\widetilde{h}$ can be interpreted as an artificial magnetic field in a non-interacting model. Performing the flow makes the real parts of the self-energy move closer to one another, in other words the artificial magnetic field decreases, i.e. the symmetry breaking is suppressed. This mechanism does, however, not fully restore spin symmetry. The reason for this is a divergence on the right-hand side of the flow equation at a particular value of $\lambda$. It originates from the term $A_{2}^{\sigma}$ of Eq. (B) which is given by $-\widetilde{\Psi}^{\mathrm{d} \sigma}(\omega=$ $0)=U \int_{-\infty}^{\infty} \frac{d \nu}{2 \pi} \bar{G}_{\bar{\sigma}}^{\lambda}(\nu)^{2}$, where $U$ denotes the bare interaction. If $\left|A_{2}^{\sigma}\right|=1$, the denominator in Eq. (B4) leads to a divergence which corresponds to the divergence that occurs in the RPA-like first factor $\left(I+U \cdot \bar{\Pi}_{\lambda}\right)^{\text {inv }}$ discussed in Sec. VID. For the unrestricted Hartree-Fock solution (i.e. at the beginning of the flow), one finds that $\widetilde{h}$ is sufficiently large, as to ensure $\left|A_{2}^{\sigma}\right|<1$. Now, as the symmetry restoring effect predicted by Ref. 19 occurs, $\widetilde{h}$ is effectively reduced, bringing $\left|A_{2}^{\sigma}\right|$ closer to its critical value 1 . When $\left|A_{2}^{\sigma}\right|=1$ is reached, the flow 
equation cannot be integrated any further. This is what happens for $U \approx U_{\text {crit }} \ldots 8 \Gamma$ for the effective mass, the charge susceptibility and the spin susceptibility. Values can be calculated for $U / \Gamma>8$ because then the restoring effect does not cause $\left|A_{2}^{\sigma}\right|=1$ for any $\lambda \leq 1$. However, the spurious spin symmetry breaking is not lifted in this case and the results are not trustworthy as explained in Sec. VID.

Analogous considerations can be applied to the restricted MUF. The restricted Hartree-Fock solution does not lead to spin symmetry breaking, i.e. it corresponds to a vanishing artificial magnetic field $\widetilde{h}=0$. Thus, $\left|A_{2}^{\sigma}\right|$ is greater than 1 at the beginning of the flow if $U>U_{\text {crit }}$. This seems to be dubious at first sight, because in the RPA-like factor $\left(I+U \cdot \bar{\Pi}_{\lambda}\right)$ inv discussed in Sec. VID this would correspond to a series of questionable convergence. However, such a series also occurs in the HartreeFock initial condition. In order to be able to renormalize this initially present contribution to the self-energy, the flow equation must contain such a term. Technically, it does not produce a divergence unless $\left|A_{2}^{\sigma}\right|=1$. If $\bar{\Sigma}_{\sigma, \lambda}^{\mathrm{C}}=\bar{\Sigma}_{\bar{\sigma}, \lambda}^{\mathrm{C}}$ (and $A_{i}^{\sigma}=A_{i}^{\bar{\sigma}}$ ) as it is the case for the restricted MUF scheme as long as $B=0$, even this point, namely $A_{2}^{\sigma}=-1$, can be crossed in the flow without the occurrence of a divergence. In order to do this in a numerically stable way, Eq. (B4) is rewritten

$$
\dot{\bar{\Sigma}}^{\lambda, \mathrm{C}}=\frac{A_{1}}{1-A_{2}} .
$$

This explains why a numerical solution of the restricted MUF flow equations remains possible even beyond $U_{\text {crit }}$ except in the presence of an external magnetic field. A small magnetic field is required for the numerical computation of the spin susceptibility which is thus not accessible for $U>U_{\text {crit }}$.

When working with the CF and CUF schemes, we know that some integrands will be zero in certain integration regions due to the step function in the propagator. We take this into account and change integration limits such that we integrate only over regions where the integrand is non-zero. The integration limits must be updated in each step of the flow because the step function in the free propagator directly depends on the flow parameter.

\section{Appendix C: Details on the calculation of the occupancy from the grand potential}

In this appendix, we provide $\Delta \bar{\Omega}$ for the cfRG and FLEX methods as well as $\dot{\bar{\Omega}}_{\lambda}$ for the PUF, StUF and MUF schemes.

The conserving case. We find

$$
\begin{aligned}
\Delta \bar{\Omega} & =\bar{\Omega}-\left.\bar{\Omega}\right|_{U^{(1)}=0=U^{(2)}} \\
& =\frac{1}{2 \beta} \operatorname{tr} \ln \left(\bar{G} C^{-1}\right)-\frac{1}{2 \beta} \operatorname{tr}\left[\left(\bar{\Sigma}+U^{(1)}\right) \bar{G}\right]+\frac{1}{\beta} \bar{\Phi}
\end{aligned}
$$

in which $\bar{\Phi}$ is given by

$$
\begin{aligned}
\bar{\Phi}=\eta_{0} \operatorname{Tr}(U \cdot \bar{\Pi})+\eta_{1} \operatorname{Tr} & {[U \cdot \bar{\Pi} \cdot U \cdot \bar{\Pi}] } \\
& +\eta_{2} \operatorname{TrLn}(I+U \cdot \bar{\Pi}) .
\end{aligned}
$$

Here, we defined some prefactors which must be chosen as $\eta_{0}=-\frac{1}{4}, \eta_{1}=\frac{1}{6}$ and $\eta_{2}=\frac{1}{2}$ for FLEX and as $\eta_{0}=\frac{1}{12}$, $\eta_{1}=0$ and $\eta_{2}=\frac{1}{6}$ for cfRG. The logarithmic expressions are defined via their series expansions

$$
\begin{aligned}
\operatorname{trln}\left(\bar{G} C^{-1}\right) & =-\operatorname{trln}\left[\left(C^{-1}-\bar{\Sigma}\right) C\right] \\
& =-\operatorname{tr} \sum_{k=1}^{\infty} \frac{(-1)^{k+1}}{k}(-\bar{\Sigma} C)^{k} \\
\operatorname{TrLn}(I+U \cdot \bar{\Pi}) & =\sum_{k=1}^{\infty} \frac{(-1)^{k+1}}{k} \operatorname{Tr}\left[(U \cdot \Pi)^{k}\right] .
\end{aligned}
$$

In each series, a convergence factor must be taken into account in the lowest contribution. Overall one finds

$$
\begin{aligned}
& \Delta \bar{\Omega}= \\
& \sum_{\sigma} \int_{0}^{\infty} \frac{d \nu}{\pi}\left\{\ln \left|\bar{G}_{\sigma}(\nu) C_{\sigma}^{-1}(-\nu)\right|-\operatorname{Re}\left[\bar{G}_{\sigma}(\nu) \bar{\Sigma}_{\sigma}^{\mathrm{D}}(\nu)^{*}\right]\right\} \\
& +\frac{1}{2} \sum_{\sigma} \bar{\Sigma}_{\sigma}^{\mathrm{C}}-\sum_{\sigma}\left(\bar{\Sigma}_{\sigma}^{\mathrm{C}}+\frac{U}{2}\right) n_{\sigma}+\left(\eta_{0}+\eta_{2}\right) 4 U n_{\uparrow} n_{\downarrow} \\
& -\eta_{2} 4 U\left(n_{\uparrow}-\frac{1}{2}\right)\left(n_{\downarrow}-\frac{1}{2}\right) \\
& +2 \eta_{1} \int_{0}^{\infty} \frac{d \omega}{\pi} \operatorname{Re}\left\{\widetilde{\Psi}^{\mathrm{p}}(\omega)^{2}+\widetilde{\Psi}^{\mathrm{d} \downarrow}(\omega) \widetilde{\Psi}^{\mathrm{d} \uparrow}(\omega)+\widetilde{\Psi}^{\mathrm{x} \uparrow}(\omega)^{2}\right\} \\
& +2 \eta_{2} \int_{0}^{\infty} \frac{d \omega}{\pi}\left\{\ln \left|1+\widetilde{\Psi}^{\mathrm{p}}(\omega)\right|+\ln \left|1+\widetilde{\Psi}^{\mathrm{x} \uparrow}(\omega)\right|\right. \\
& \left.+\frac{1}{2} \ln \left(1-\widetilde{\Psi}^{\mathrm{d} \uparrow}(\omega) \widetilde{\Psi}^{\mathrm{d} \downarrow}(\omega)\right)\right\} .
\end{aligned}
$$

The non-conserving case. In Ref. 20, it was shown that $\dot{\bar{\Omega}}_{\lambda}=\overline{\dot{\Gamma}}_{\lambda} / \beta$. This still holds. However, this is not equal to $\bar{\Phi}_{\lambda} / \beta$ any more in the PUF and StUF schemes because $U^{(1)}$ in Eq. (77) acquires a $\lambda$-dependence. We thus have

$$
\begin{aligned}
\dot{\bar{\Omega}}_{\lambda}^{\mathrm{PUF}} & =\frac{1}{\beta} \dot{\Phi}_{\lambda}^{\mathrm{PUF}}+\frac{1}{\beta} \dot{U}_{\lambda}^{(1)} \cdot \bar{G}_{\lambda} \\
& =\frac{1}{3 ! \beta} \operatorname{Tr}\left(\dot{U}_{\lambda}^{(2)} \cdot\left[\frac{3}{2} \bar{\Pi}_{\lambda}-\bar{\Pi}_{\lambda} \cdot \bar{\Upsilon}_{\lambda}\right]\right)+\frac{1}{\beta} \dot{U}_{\lambda}^{(1)} \cdot \bar{G}_{\lambda}
\end{aligned}
$$

for the PUF approximation. Here, we used Eq. (106) of Ref. 20, Explicitly, this means

$$
\begin{aligned}
& \dot{\bar{\Omega}}_{\lambda}^{\mathrm{PUF}}= \\
& \dot{U}_{\lambda} \int_{0}^{\infty} \frac{d \nu}{\pi} \operatorname{Re}\left[\bar{G}_{\uparrow}(\nu)\right] \int_{0}^{\infty} \frac{d \nu}{\pi} \operatorname{Re}\left[\bar{G}_{\downarrow}(\nu)\right]-\frac{\dot{U}_{\lambda}}{4} \\
& -\frac{1}{3 !} \int_{-\infty}^{\infty} \frac{d \omega}{2 \pi}\left\{2 \operatorname{Re}\left[\widetilde{\Psi}_{\lambda \bullet}^{\mathrm{p}}(\omega) \widetilde{\Upsilon}_{\lambda}^{\mathrm{p}}(\omega)+\widetilde{\Psi}_{\lambda \bullet}^{\mathrm{x} \uparrow}(\omega) \widetilde{\Upsilon}_{\lambda}^{\mathrm{x} \uparrow}(\omega)\right]\right. \\
& \left.+\widetilde{\Psi}_{\lambda \bullet}^{\mathrm{d} \uparrow}(\omega) \widetilde{\Upsilon}_{\lambda}^{\mathrm{d} \downarrow}(\omega)+\widetilde{\Psi}_{\lambda \bullet}^{\mathrm{d} \downarrow}(\omega) \widetilde{\Upsilon}_{\lambda}^{\mathrm{d} \uparrow}(\omega)\right\} .
\end{aligned}
$$


Here, we introduced $\widetilde{\Psi}_{\lambda \bullet}^{i}=\left.\widetilde{\Psi}_{\lambda}^{i}\right|_{U_{\lambda} \rightarrow \dot{U}_{\lambda}}$. Similarly, we obtain

$$
\dot{\bar{\Omega}}_{\lambda}^{\mathrm{StUF}}=\dot{U}_{\lambda} \int_{0}^{\infty} \frac{d \nu}{\pi} \operatorname{Re}\left[\bar{G}_{\uparrow}(\nu)\right] \int_{0}^{\infty} \frac{d \nu}{\pi} \operatorname{Re}\left[\bar{G}_{\downarrow}(\nu)\right]-\frac{\dot{U}_{\lambda}}{4} .
$$

For the MUF approximation we do not have an additional term and we can simply use Eq. (108) of Ref. 20 to find

$$
\begin{array}{r}
\dot{\bar{\Omega}}_{\lambda}^{\mathrm{MUF}}=-\frac{1}{3 ! \beta} \operatorname{Tr}\left(\dot{U}_{\lambda}^{(2)} \cdot \bar{\Pi}_{\lambda} \cdot \bar{\Upsilon}_{\lambda}\right) \\
=-\frac{1}{3 !} \int_{-\infty}^{\infty} \frac{d \omega}{2 \pi}\left\{2 \operatorname{Re}\left[\widetilde{\Psi}_{\lambda \bullet}^{\mathrm{p}}(\omega) \widetilde{\Upsilon}_{\lambda}^{\mathrm{p}}(\omega)+\widetilde{\Psi}_{\lambda \bullet}^{\mathrm{x} \uparrow}(\omega) \widetilde{\Upsilon}_{\lambda}^{\mathrm{x} \uparrow}(\omega)\right]\right. \\
\left.+\widetilde{\Psi}_{\lambda \bullet}^{\mathrm{d} \uparrow}(\omega) \widetilde{\Upsilon}_{\lambda}^{\mathrm{d} \downarrow}(\omega)+\widetilde{\Psi}_{\lambda \bullet}^{\mathrm{d} \downarrow}(\omega) \widetilde{\Upsilon}_{\lambda}^{\mathrm{d} \uparrow}(\omega)\right\} .
\end{array}
$$

1 G. Baym and L. P. Kadanoff, Phys. Rev. 124, 287 (1961), URL http://link.aps.org/doi/10.1103/PhysRev.124. 287

${ }^{2}$ P. W. Anderson, Phys. Rev. 124, 41 (1961), URL http: //link.aps.org/doi/10.1103/PhysRev.124.41

${ }^{3}$ R. Shankar, Rev. Mod. Phys. 66, 129 (1994), URL http: //link.aps.org/doi/10.1103/RevModPhys.66.129

${ }^{4}$ G. Baym, Phys. Rev. 127, 1391 (1962), URL http:// link.aps.org/doi/10.1103/PhysRev.127.1391

5 P. C. E. Stamp, Journal of Physics F: Metal Physics 15, 1829 (1985), URL http://stacks.iop.org/0305-4608/ $15 / i=9 / a=005$

${ }^{6}$ N. E. Bickers and S. R. White, Phys. Rev. B 43, 8044 (1991), URL http://link.aps.org/doi/10.1103/ PhysRevB.43.8044

${ }^{7}$ N. Bickers, Int. J. Mod. Phys. B 5, 253 (1991), URL http://www.worldscientific.com/doi/abs/10.1142/ S021797929100016X.

8 A. A. Katanin, Phys. Rev. B 70, 115109 (2004), URL http://link.aps.org/doi/10.1103/PhysRevB.70. 115109

${ }^{9}$ W. Metzner, M. Salmhofer, C. Honerkamp, V. Meden, and K. Schönhammer, Rev. Mod. Phys. 84, 299 (2012), URL http://link.aps.org/doi/10.1103/RevModPhys.84.299

10 T. Enss, Ph.D. thesis, Max-Planck-Institut für Festkörperforschung (2005).

11 F. Schütz, L. Bartosch, and P. Kopietz, Phys. Rev. B 72, 035107 (2005), URL http://link .aps .org/doi/10.1103/ PhysRevB.72.035107.

${ }^{12}$ L. Bartosch, P. Kopietz, and A. Ferraz, Phys. Rev. B 80, 104514 (2009), URL http://link .aps .org/doi/10.1103/ PhysRevB.80.104514.

13 S. Streib, A. Isidori, and P. Kopietz, Phys. Rev. B 87, 201107 (2013), URL http://link .aps .org/doi/10.1103/ PhysRevB.87.201107.

14 J. M. Luttinger and J. C. Ward, Phys. Rev. 118, 1417 (1960), URL http://link.aps.org/doi/10.1103/ PhysRev.118.1417.

15 J.-P. Blaizot, J. M. Pawlowski, and U. Reinosa, Phys. Lett. B 696, 523 (2011), ISSN 0370-2693, URL http://www.sciencedirect.com/science/article/pii/ S0370269310014619.
16 J. Berges, S. Borsnyi, U. Reinosa, and J. Serreau, Annals of Physics 320, 344 (2005), ISSN 00034916, URL http://www.sciencedirect.com/science/ article/pii/S0003491605001156

Ir M. E. Carrington, W.-J. Fu, D. Pickering, and J. W. Pulver, Phys. Rev. D 91, 025003 (2015), URL http: //link.aps.org/doi/10.1103/PhysRevD.91.025003

18 N. Dupuis, The European Physical Journal B - Condensed Matter and Complex Systems 48, 319 (2005), ISSN 1434-6028, URL http://dx.doi.org/10.1140/ epjb/e2005-00409-7

${ }^{19}$ N. Dupuis, Phys. Rev. B 89, 035113 (2014), URL http: //link.aps.org/doi/10.1103/PhysRevB.89.035113.

20 J. F. Rentrop, S. G. Jakobs, and V. Meden, Journal of Physics A: Mathematical and Theoretical 48, 145002 (2015), URL http://stacks.iop.org/1751-8121/48/i= $14 / \mathrm{a}=145002$

21 N. E. Bickers, D. J. Scalapino, and S. R. White, Phys. Rev. Lett. 62, 961 (1989), URL http://link.aps.org/doi/10. 1103/PhysRevLett.62.961.

${ }^{22}$ D. R. Hamann, Phys. Rev. 186, 549 (1969), URL http: //link.aps.org/doi/10.1103/PhysRev.186.549.

${ }^{23}$ C. De Dominicis and P. C. Martin, J. Math. Phys. 5, 14 (1964), URL http://scitation.aip.org/content/aip/ journal/jmp/5/1/10.1063/1.1704062.

${ }^{24}$ C. De Dominicis and P. C. Martin, J. Math. Phys. 5, 31 (1964), URL http://scitation.aip.org/content/aip/ journal/jmp/5/1/10.1063/1.1704064.

25 C. Wetterich, Phys. Rev. B 75, 085102 (2007), URL http: //link.aps.org/doi/10.1103/PhysRevB.75.085102.

${ }^{20}$ K. B. Dave, P. W. Phillips, and C. L. Kane, Phys. Rev. Lett. 110, 090403 (2013), URL http://link.aps.org/ doi/10.1103/PhysRevLett.110.090403

${ }^{2 r}$ E. Kozik, M. Ferrero, and A. Georges, Phys. Rev. Lett. 114, 156402 (2015), URL http://link.aps .org/doi/10. 1103/PhysRevLett.114.156402

28 A. Stan, P. Romaniello, S. Rigamonti, L. Reining, and J. A. Berger, New J. Phys. 17, 093045 (2015), URL http: //stacks.iop.org/1367-2630/17/i=9/a=093045.

${ }^{2 y}$ R. Rossi and F. Werner, J. Phys. A: Math. Theor. 48, 485202 (2015), URL http://stacks .iop.org/1751-8121/ $48 / i=48 / a=485202$. 
${ }^{30}$ C. Karrasch, R. Hedden, R. Peters, T. Pruschke, K. Schönhammer, and V. Meden, Journal of Physics: Condensed Matter 20, 345205 (2008), URL http://stacks. iop.org/0953-8984/20/i=34/a=345205.

31 J. S. Langer and V. Ambegaokar, Phys. Rev. 121, 1090 (1961), URL http://link.aps.org/doi/10.1103/ PhysRev.121.1090

32 J. M. Luttinger, Phys. Rev. 119, 1153 (1960), URL http: //link.aps.org/doi/10.1103/PhysRev.119.1153.

33 A. Rosch, Eur. Phys. J. B 59, 495 (2007), URL http: //dx.doi.org/10.1140/epjb/e2007-00312-3

${ }^{4}$ K. Yamada, Progress of Theoretical Physics 53, 970 (1975), URL http://ptp.oxfordjournals.org/content/ 53/4/970. abstract.

35 N. Bickers and D. Scalapino, Ann. Phys. (N.Y.) 193, 206 (1989), URL http://www.sciencedirect.com/science/ article/pii/000349168990359X.

36 D. Manske, Theory of Unconventional Superconductors: Cooper-Pairing Mediated by Spin Excitations, no. 202 in Springer Tracts in Modern Physics (Springer, Berlin, New York, 2004), ISBN 3-540-21229-9.

37 T. Maier, M. Jarrell, T. Pruschke, and M. H. Hettler, Rev. Mod. Phys. 77, 1027 (2005), URL http://link.aps .org/ doi/10.1103/RevModPhys.77.1027.

38 J. A. White, Phys. Rev. B 45, 1100 (1992), URL http: //link.aps.org/doi/10.1103/PhysRevB.45.1100

39 S. Andergassen, T. Enss, V. Meden, W. Metzner, U. Schollwöck, and K. Schönhammer, Phys. Rev. B 70, 075102 (2004), URL http://link.aps.org/doi/10.1103/ PhysRevB.70.075102

${ }^{40}$ A. Hewson, The Kondo Problem to Heavy Fermions (Cam- bridge University Press, New York, N.Y., 1993).

41 C. Karrasch, T. Enss, and V. Meden, Phys. Rev. B 73, 235337 (2006), URL http://link.aps .org/doi/10.1103/ PhysRevB.73.235337.

42 V. Zlatić and B. Horvatić, Phys. Rev. B 28, 6904 (1983), URL http://link.aps.org/doi/10.1103/PhysRevB.28. 6904

43 U. Gerland, J. von Delft, T. A. Costi, and Y. Oreg, Phys. Rev. Lett. 84, 3710 (2000), URL http://link.aps.org/ doi/10.1103/PhysRevLett.84.3710

${ }^{44}$ H. Suhl, Phys. Rev. Lett. 19, 442 (1967), URL http:// link.aps.org/doi/10.1103/PhysRevLett.19.442

45 J. F. Rentrop, Ph.D. thesis, RWTH Aachen (in preparation).

46 M. Kinza and C. Honerkamp, Phys. Rev. B 92, 045113 (2015), URL http://link .aps .org/doi/10.1103/ PhysRevB.92.045113.

4 This conserving 2PI functional RG scheme should not be confused with the "constrained functional RG" proposed by Kinza and Honerkamp ${ }^{\frac{46}{6}}$ which they also abbreviate as "cfRG".

48 There is a minor deviation for the charge susceptibility at large values of $U / \Gamma$. In Fig. 5 of Ref. 38, the three points plotted for the largest $U / \Gamma$ seem to indicate the presence of a very slight shoulder; our data shown in Fig. 2(b) does not do so. We find agreement to the middle one of these three points (the one at $U / \pi \Gamma \approx 2.3$ ) but slight deviations from the other two points. We suspect that those two points of White are not fully converged. 